\title{
FUSION SYSTEMS AND AMALGAMS
}

\author{
KASPER K. S. ANDERSEN, BOB OLIVER, AND JOANA VENTURA
}

\begin{abstract}
We study reduced fusion systems from the point of view of their essential subgroups, using the classification by Goldschmidt and Fan of amalgams of prime index to analyze certain pairs of such subgroups. Our results are applied here to study reduced fusion systems over 2 -groups of order at most 64 , and also reduced fusion systems over 2-groups having abelian subgroups of index two. More applications are given in later papers.
\end{abstract}

A saturated fusion system over a finite $p$-group $S$ is a category whose objects are the subgroups of $S$, whose morphisms are monomorphisms between subgroups, and which satisfy certain axioms first formulated by Puig [Pg2] and motivated by conjugacy relations among $p$-subgroups of a given finite group. A saturated fusion system is reduced if it has no proper normal subsystem of $p$-power index, no proper normal subsystem of index prime to $p$, and no nontrivial normal $p$-subgroup. (All three of these concepts are defined by analogy with finite groups.) Reduced fusion systems need not be simple, in that they can have proper nontrivial normal subsystems. They were introduced by us in [AOV] as forming a class of fusion systems which is small enough to be manageable, but still large enough to detect any fusion systems (reduced or not) which are "exotic" (not defined via conjugacy relations in any finite group).

When $G$ is a finite group and $S \in \operatorname{Syl}_{p}(G)$, the version of Alperin's fusion theorem shown by Goldschmidt [Gd1] says that all $G$-conjugacy relations among subgroups of $S$ are generated by $\operatorname{Aut}_{G}(S)$ (automorphisms induced by conjugation in $G$ ), together with $\operatorname{Aut}_{G}(P)$ for certain "essential" proper subgroups of $S$, and restrictions of such automorphisms. There is a version of this result for abstract fusion systems (see Theorem 1.2), which says that a fusion system $\mathcal{F}$ is generated by $\mathcal{F}$-automorphisms of $\mathcal{F}$-essential subgroups (Definition 1.1). Our goal in this and our other papers is to study, and to classify in certain cases, reduced fusion systems from the point of view of their essential subgroups and generating automorphisms.

This point of view was introduced in $[\mathrm{OV}]$, where two of us described how fusion systems over a given 2-group $S$ could be classified by first listing the subgroups of $S$ which potentially could be essential, using Bender's theorem on groups with strongly embedded subgroups. When we try to extend those methods to larger classes of groups, it is useful to search for pairs of essential subgroups via theorems of Goldschmidt and Fan classifying certain types of amalgams.

2010 Mathematics Subject Classification. Primary 20D20. Secondary 20E06, 20E45.

Key words and phrases. Finite groups, fusion, amalgams, fusion systems.

K. K. S. Andersen was partially supported by the Danish National Research Foundation (DNRF) through the Centre for Symmetry and Deformation.

B. Oliver is partially supported by UMR 7539 of the CNRS, and by project ANR BLAN08-2_338236, HGRT.

J. Ventura was partially supported by FCT through program POCI 2010/FEDER and project PTDC/MAT/098317/2008. 
The situation we want to study is the following. Assume $\mathcal{F}$ is a saturated fusion system over a finite 2-group $S$, and $P_{1}, P_{2}<S$ are $\mathcal{F}$-essential subgroups of index two in their normalizer. In addition, we assume either that $P_{1}$ and $P_{2}$ have index two in $S$, or that $N_{S}\left(P_{1}\right)=N_{S}\left(P_{2}\right)<S$ and $P_{1}, P_{2}$ are $S$-conjugate to each other. Set $P=P_{1} P_{2}=N_{S}\left(P_{i}\right)$. Then there are finite groups $G_{i}>P$ such that $P_{i} \unlhd G_{i}$, $G_{i} / P_{i} \cong D_{2 p_{i}}$ for some odd prime $p_{i}$, and $\operatorname{Aut}_{G_{i}}\left(P_{i}\right) \leq \operatorname{Aut}_{\mathcal{F}}\left(P_{i}\right)$. By applying the classifications of amalgams by Goldschmidt and Fan to the triple $\left(G_{1}>P<G_{2}\right)$, we get information about $S$ and the $P_{i}$. In this paper, we only deal with certain cases (see Theorems 4.5 and 4.6), but these are the cases which occur most often in "small" examples.

Applications of these results are given in Section 5. The reduced fusion systems over 2-groups of order at most 32, and the groups of order 64 which support reduced fusion systems, are all listed in Theorems 5.3 and 5.4, respectively. These are preceded by Propositions 5.1 and 5.2, which list various conditions on a reduced fusion system over a 2-group $S$ which imply that $S$ is dihedral of order at least 8, semidihedral of order at least 16, or a wreath product $C_{2^{n}} \nmid C_{2}$ for $n \geq 2$. Furthermore, in these cases, $\mathcal{F}$ is isomorphic to the fusion system of $P S L_{2}(q)$ for some $q \equiv \pm 1(\bmod 8)$, or of $P S L_{3}(q)$ for some odd $q$. For example, by Proposition 5.2(a,b,c,e), these conclusions hold whenever $\mathcal{F}$ is a reduced fusion system over a 2 -group $S$, where either

- $S$ contains an abelian subgroup of index two; or

- $[S, S]$ is cyclic; or

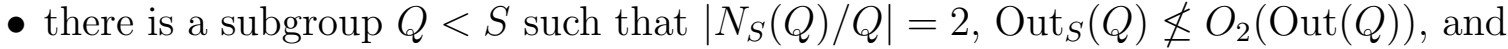
either $Q$ is abelian or $|Q| \leq 16$.

These results are applied in a later paper by the same authors, where we combine them with a computer search to list reduced fusion systems over 2-groups of order at most $2^{9}$. They have also been applied by the second author when classifying reduced fusion systems over 2-groups of sectional rank at most four.

Notation: For any group $G$, we let $G^{\text {ab }}=G /[G, G]$ denote its abelianization. Also, $C_{n}$ denotes a (multiplicative) cyclic group of order $n$, and $D_{2^{m}}, S D_{2^{m}}$, and $Q_{2^{m}}$ denote dihedral, semidihedral, and quaternion groups of order $2^{m}$. As usual, when $P$ is a finite $p$-group for some prime $p$, then $\Omega_{1}(P)=\left\langle g \in P \mid g^{p}=1\right\rangle$, and $\operatorname{Fr}(P)=\left\langle a^{p},[a, b] \mid a, b \in P\right\rangle$ (the Frattini subgroup). For any finite group $G, O_{p}(G)$ is the largest normal $p$-subgroup of $G$, and $O^{p}(G)$ is the smallest normal subgroup of $p$-power index.

When $G$ acts on a group $X$, we let $C_{X}(G)$ be the subgroup of elements of $X$ fixed by $G$. When $A \subseteq G$ and $B \subseteq X$ are subsets, we set $[A, B]=\left\langle g(x) x^{-1} \mid g \in A, x \in B\right\rangle$. When $g, h$ are elements of any group $G$, we write their commutator $[g, h]=g h g^{-1} h^{-1}$.

\section{SATURATED FUSION SYSTEMS}

When $G$ is a finite group and $S \in \operatorname{Syl}_{p}(G)$, the fusion system of $G$ over $S$ is the category $\mathcal{F}_{S}(G)$ whose objects are the subgroups of $S$, and where $\operatorname{Mor}_{\mathcal{F}_{S}(G)}(P, Q)=$ $\operatorname{Hom}_{G}(P, Q)$ is the set of monomorphisms from $P$ to $Q$ induced by conjugation in $G$. An abstract fusion system $\mathcal{F}$ over a finite $p$-group $S$ is a category whose objects are the subgroups of $S$, whose morphisms are monomorphisms of groups including all those induced by conjugation in $S$, and where for each $\varphi \in \operatorname{Hom}_{\mathcal{F}}(P, Q)=\operatorname{Mor}_{\mathcal{F}}(P, Q), \varphi$ restricts to an $\mathcal{F}$-isomorphism from $P$ to $\varphi(P) \leq Q$. A fusion system is saturated if 
it satisfies certain additional conditions. Rather than listing those conditions here, we refer to [AKO, Definition I.2.2] or our earlier paper [AOV].

In particular, for any finite $G$ with $S \in \operatorname{Syl}_{p}(G), \mathcal{F}_{S}(G)$ is a saturated fusion system (cf. [AKO, Theorem I.2.3]). An abstract fusion system $\mathcal{F}$ over $S$ is called realizable if $\mathcal{F}=\mathcal{F}_{S}(G)$ for some finite group $G$ with $S \in \operatorname{Syl}_{p}(G)$, and is called exotic otherwise.

If $G$ is a finite group and $p$ is a prime, then a proper subgroup $H<G$ is strongly p-embedded in $G$ if $p|| H \mid$, and for each $g \in G \backslash H, p \nmid\left|H \cap g H^{-1}\right|$. We refer to [AKO, Proposition A.7] for a very brief survey of some of the properties of strongly $p$-embedded subgroups, and to $[\mathrm{A} 1, \S 46]$ or $[$ Sz2, $\S 6.4]$ for more details.

Definition 1.1. Fix a prime $p$, a finite $p$-group $S$, and a saturated fusion system $\mathcal{F}$ over $S$. Let $P \leq S$ be any subgroup. Set $\operatorname{Out}_{\mathcal{F}}(P)=\operatorname{Aut}_{\mathcal{F}}(P) / \operatorname{Inn}(P)$.

- $P^{\mathcal{F}}$ denotes the set of subgroups of $S$ which are $\mathcal{F}$-conjugate to $P$; i.e., isomorphic to $P$ in the category $\mathcal{F}$. For each $g \in S, g^{\mathcal{F}}$ denotes the $\mathcal{F}$-conjugacy class of $g$.

- $P$ is fully normalized in $\mathcal{F}$ if $\left|N_{S}(P)\right| \geq\left|N_{S}(Q)\right|$ for each $Q \in P^{\mathcal{F}}$.

- $P$ is $\mathcal{F}$-centric if $C_{S}(Q)=Z(Q)$ for all $Q \in P^{\mathcal{F}}$.

- $P$ is $\mathcal{F}$-essential if $P<S, P$ is $\mathcal{F}$-centric and fully normalized in $\mathcal{F}$, and $\operatorname{Out}_{\mathcal{F}}(P)$ contains a strongly p-embedded subgroup.

- $P$ is normal in $\mathcal{F}(P \unlhd \mathcal{F})$ if $P \unlhd S$ and every morphism $\varphi \in \operatorname{Hom}_{\mathcal{F}}(Q, R)$ in $\mathcal{F}$ extends to a morphism $\bar{\varphi} \in \operatorname{Hom}_{\mathcal{F}}(P Q, P R)$ such that $\bar{\varphi}(P)=P$.

- $O_{p}(\mathcal{F})$ denotes the largest subgroup of $S$ which is normal in $\mathcal{F}$.

- $N_{\mathcal{F}}(P) \subseteq \mathcal{F}$ denotes the largest fusion subsystem over $N_{S}(P)$ (i.e., the largest subcategory of $\mathcal{F}$ which is a fusion system over $\left.N_{S}(P)\right)$ which contains $P$ as normal subgroup.

- For each $\varphi \in \operatorname{Aut}(S), \varphi \mathcal{F} \varphi^{-1}$ is the fusion system over $S$ defined by

$$
\begin{aligned}
& \operatorname{Hom}_{\varphi \mathcal{F} \varphi^{-1}}(P, Q)=\left\{\left(\left.\varphi\right|_{\varphi^{-1}(Q)}\right) \circ \psi \circ\left(\left.\varphi\right|_{\varphi^{-1}(P)}\right)^{-1} \mid \psi \in \operatorname{Hom}_{\mathcal{F}}\left(\varphi^{-1}(P), \varphi^{-1}(Q)\right)\right\} \\
& \quad \text { for all } P, Q \leq S .
\end{aligned}
$$

It follows immediately from the definition of a normal subgroup in $\mathcal{F}$ that the maximal subgroup $O_{p}(\mathcal{F}) \unlhd \mathcal{F}$ is uniquely defined. The notation is, of course, chosen by analogy with that for finite groups.

We now look at essential subgroups of a fusion system.

Theorem 1.2. Let $\mathcal{F}$ be a saturated fusion system over a finite p-group $S$. Then each morphism in $\mathcal{F}$ is a composite of restrictions of morphisms in $\operatorname{Aut}_{\mathcal{F}}(S)$, and of morphisms in $O^{p^{\prime}}\left(\operatorname{Aut}_{\mathcal{F}}(P)\right)$ for $\mathcal{F}$-essential subgroups $P \leq S$.

Proof. See, e.g., [O1, Proposition 1.10(a,b)]. In fact, a proper subgroup $P<S$ fully normalized in $\mathcal{F}$ is $\mathcal{F}$-essential exactly when $\operatorname{Aut}_{\mathcal{F}}(P)$ is not generated by restrictions of morphisms between strictly larger subgroups of $S$. (See [OV, Proposition 2.5] or [AKO, Proposition I.3.3(b)] for more details.)

The next proposition follows easily from Theorem 1.2, together with the definition of a normal $p$-subgroup. 
Proposition 1.3. Let $\mathcal{F}$ be a saturated fusion system over a finite $p$-group $S$, and fix $Q \leq S$. Assume, whenever $P=S$ or $P$ is $\mathcal{F}$-essential, that $Q \leq P$ and each $\alpha \in \operatorname{Aut}_{\mathcal{F}}(P)$ sends $Q$ to itself. Then $Q \unlhd \mathcal{F}$.

Proof. See, e.g., [AKO, Proposition I.4.5] for details.

The next two lemmas are our main tools for detecting essential subgroups, or rather, for proving that certain subgroups are not essential.

Lemma 1.4 ([OV, Lemma 3.4]). Fix a prime $p$, a finite p-group $S$, a subgroup $P \leq S$, and a characteristic subgroup $\Theta \leq P$. Assume there is $g \in N_{S}(P) \backslash P$ such that

(a) $[g, P] \leq \Theta \cdot \operatorname{Fr}(P)$, and

(b) $[g, \Theta] \leq \operatorname{Fr}(P)$.

Then $c_{g} \in O_{p}(\operatorname{Aut}(P))$. Hence $P$ is not $\mathcal{F}$-essential for any saturated fusion system $\mathcal{F}$ over $S$.

In fact, [OV, Lemma 3.4] is stated in terms of "(semi)critical subgroups" of a finite $p$-group $S$ rather than essential subgroups. We refer to [OV, Definition 3.1] for the definition of critical subgroups, and just note here that by [OV, Proposition 3.2], each $\mathcal{F}$-essential subgroup (for any saturated fusion system $\mathcal{F}$ over $S$ ) is critical in $S$. This remark also applies to the next lemma, which is a special case of [OV, Proposition $3.3(\mathrm{c})]$.

Lemma 1.5. Let $S$ be a finite 2-group. Assume that $P \leq S$ is $\mathcal{F}$-essential for some saturated fusion system $\mathcal{F}$ over $S$, and also that $\left|N_{S}(P) / P\right| \geq 4$. Then $\operatorname{rk}(P / \operatorname{Fr}(P)) \geq$ 4 , and $\operatorname{rk}([s, P / \operatorname{Fr}(P)]) \geq 2$ for all $s \in N_{S}(P) \backslash P$.

We next recall the definitions of the focal and hyperfocal subgroups of a saturated fusion system, defined by analogy with the finite group case.

Definition 1.6. Let $\mathcal{F}$ be a saturated fusion system over a finite p-group $S$. The focal subgroup of $\mathcal{F}$ is the subgroup

$$
\begin{aligned}
\mathfrak{f o c}(\mathcal{F}) & \left.\stackrel{\text { def }}{=}\left\langle g^{-1} h\right| g, h \in S \text { and } h \in g^{\mathcal{F}}\right\rangle \\
& \left.=\left\langle g^{-1} \alpha(g)\right| g \in P \leq S, P=S \text { or } P \text { is } \mathcal{F} \text {-essential, } \alpha \in \operatorname{Aut}_{\mathcal{F}}(P)\right\rangle .
\end{aligned}
$$

The hyperfocal subgroup of $\mathcal{F}$ is the subgroup

$$
\mathfrak{h} \mathfrak{y} \mathfrak{p}(\mathcal{F})=\left\langle g^{-1} \alpha(g) \mid g \in P \leq S, \alpha \in O^{p}\left(\operatorname{Aut}_{\mathcal{F}}(P)\right)\right\rangle .
$$

The two definitions of $\mathfrak{f o c}(\mathcal{F})$ are equivalent by Theorem 1.2. In the definition of $\mathfrak{h y p}(\mathcal{F})$, we could equivalently restrict to automorphisms of order prime to $p$. When $\mathcal{F}=\mathcal{F}_{S}(G)$ for a finite group $G$ and $S \in \operatorname{Syl}_{p}(G)$, then $\mathfrak{f o c}(\mathcal{F})=S \cap[G, G]$ by the focal subgroup theorem (cf. [G, Theorem 7.3.4]), and $\mathfrak{h y p}(\mathcal{F})=S \cap O^{p}(G)$ by the hyperfocal theorem of Puig [Pg1, §1.1].

Next recall the following definitions from [5a2].

Definition 1.7. Let $\mathcal{F}$ be a saturated fusion system over a finite p-group $S$, and let $\mathcal{F}_{0} \subseteq \mathcal{F}$ be a saturated fusion subsystem over a subgroup $S_{0} \leq S$.

(a) $\mathcal{F}_{0}$ has p-power index in $\mathcal{F}$ if $\mathfrak{h y p}(\mathcal{F}) \leq S_{0} \leq S$, and $\operatorname{Aut}_{\mathcal{F}_{0}}(P) \geq O^{p}\left(\operatorname{Aut}_{\mathcal{F}}(P)\right)$ for all $P \leq S_{0}$. 
(b) $\mathcal{F}_{0}$ has index prime to $p$ in $\mathcal{F}$ if $S_{0}=S$, and $\operatorname{Aut}_{\mathcal{F}_{0}}(P) \geq O^{p^{\prime}}\left(\operatorname{Aut}_{\mathcal{F}}(P)\right)$ for all $P \leq S$.

By [5a2, Theorems $4.3 \& 5.4]$, each saturated fusion system $\mathcal{F}$ over a finite $p$-group $S$ contains a unique minimal saturated fusion subsystem $O^{p}(\mathcal{F})$ of $p$-power index (over $\mathfrak{h y p}(\mathcal{F})$ ), and a unique minimal saturated fusion subsystem $O^{p^{\prime}}(\mathcal{F})$ of index prime to $p$ (over $S$ ). Furthermore:

Proposition 1.8. For any saturated fusion system $\mathcal{F}$ over a finite $p$-group $S$,

$$
\mathcal{F}=O^{p}(\mathcal{F}) \Longleftrightarrow \mathfrak{h} \mathfrak{y p}(\mathcal{F})=S \Longleftrightarrow \mathfrak{f o c}(\mathcal{F})=S
$$

Proof. See, e.g., [AKO, Corollary I.7.5]. The second equivalence follows upon checking that the image of $\mathfrak{f} \mathfrak{c}(\mathcal{F})$ in $S / \mathfrak{h} \mathfrak{y} \mathfrak{p}(\mathcal{F})$ is precisely its commutator subgroup (cf. [AKO, Lemma I.7.2]).

\section{REDUCED FUSION SYSTEMS}

This paper is centered around the special class of reduced fusion systems, which are defined as follows.

Definition 2.1. A reduced fusion system is a saturated fusion system $\mathcal{F}$ such that

- $\mathcal{F}$ has no nontrivial normal p-subgroups,

- $\mathcal{F}$ has no proper subsystem of p-power index, and

- $\mathcal{F}$ has no proper subsystem of index prime to $p$.

Equivalently, $\mathcal{F}$ is reduced if $O_{p}(\mathcal{F})=1, O^{p}(\mathcal{F})=\mathcal{F}$, and $O^{p^{\prime}}(\mathcal{F})=\mathcal{F}$.

Definition 2.1 was originally formulated in $[\mathrm{AOV}]$, and was motivated by Theorems A and B in that paper. Very roughly, those theorems describe a way to "detect" exotic fusion systems while looking only at reduced fusion systems.

In this section, we give some conditions on a fusion system which are necessary for it to be reduced (equivalently, conditions which imply that it is not reduced). We begin with two very general results.

Lemma 2.2. If $\mathcal{F}$ is a reduced fusion system over a nontrivial finite 2-group $S$, and $\mathcal{E}$ is the set of $\mathcal{F}$-essential subgroups of $S$, then $|\mathcal{E}| \geq 2$ and $[S:\langle\mathcal{E}\rangle] \neq 2$.

Proof. By Proposition 1.3, $S \unlhd \mathcal{F}$ if $\mathcal{E}=\varnothing$, while $P \unlhd \mathcal{F}$ if $\mathcal{E}=\{P\}$ for some $P$. So $\mathcal{F}$ is not reduced $\left(O_{p}(\mathcal{F}) \neq 1\right)$ if $|\mathcal{E}| \leq 1$.

If $[S:\langle\mathcal{E}\rangle]=2$, then $\left[\operatorname{Aut}_{\mathcal{F}}(S), S\right] \leq\langle\mathcal{E}\rangle$ since $\operatorname{Aut}_{\mathcal{F}}(S)$ acts trivially on $S /\langle\mathcal{E}\rangle \cong C_{2}$. Since $\mathfrak{f o c}(\mathcal{F})$ is generated by $\left[\operatorname{Aut}_{\mathcal{F}}(S), S\right]$ and the $\left[\operatorname{Aut}_{\mathcal{F}}(P), P\right]$ for $P \in \mathcal{E}, \mathfrak{f o c}(\mathcal{F}) \leq$ $\langle\mathcal{E}\rangle<S$. So $\mathcal{F}$ is not reduced by Proposition 1.8.

The next proposition is a simple application of a transfer homomorphism for fusion systems.

Proposition 2.3. Let $\mathcal{F}$ be a saturated fusion system over a finite 2-group $S$.

(a) Assume there is $g \in \Omega_{1}(Z(S)) \backslash[S, S]$ such that each $\alpha \in \operatorname{Aut}_{\mathcal{F}}(S)$ sends the coset $g[S, S]$ to itself. Then $g \notin \mathfrak{f o c}(\mathcal{F})$. 
(b) More generally, let $U \unlhd S$ be such that each element of $\operatorname{Aut}_{\mathcal{F}}(S)$ sends $U$ to itself, and $U \leq[P, P]$ for each $P<S$ of index two. Assume there is $g \in S \backslash[S, S]$ such that $[g, S] \leq U, g^{2} \in U$, and each $\alpha \in \operatorname{Aut}_{\mathcal{F}}(S)$ sends the coset $g[S, S]$ to itself. Then $g \notin \mathfrak{f o c}(\mathcal{F})$.

In either case, $\mathcal{F}$ is not reduced.

Proof. We refer to $[\mathrm{AKO}, \S \mathrm{I}$.8] for some of the properties of the transfer homomorphism $\operatorname{trf}_{\mathcal{F}}: S / \mathfrak{f o c}(\mathcal{F}) \longrightarrow S^{\text {ab }}$ when $\mathcal{F}$ is a saturated fusion system over $S$. Let $[g] \in S^{\text {ab }}$ be the class of $g$. Since (a) is a special case of (b) (the case $U=1$ ), and was shown in [AKO, Corollary I.8.5], we assume $g$ satisfies the conditions of (b).

For $P<S$, let $\operatorname{trf}_{P}^{S}: S^{\mathrm{ab}} \longrightarrow P^{\mathrm{ab}}$ be the usual transfer homomorphism (cf. [AKO, Lemma I.8.1(b)]). If $[S: P]=2$, then $\operatorname{trf}_{P}^{S}([g])=\left[g^{2}\right]$ if $g \notin P$, and $\operatorname{trf}_{P}^{S}([g])=\left[g x g x^{-1}\right]$ if $g \in P$ and $x \in S \backslash P$. This follows from the construction in [AKO] upon taking coset representatives $\{1, x\}$. Since $g^{2} \in U, g x g x^{-1}=g^{2}\left[g^{-1}, x\right] \in U$, and $U \leq[P, P]$, $\operatorname{trf}_{P}^{S}([g])=1$. Since this holds for each $P<S$ of index two, $\operatorname{trf}_{P}^{S}([g])=1$ for each $P<S$ since transfers compose (cf. [AKO, Lemma I.8.1(d)]).

By assumption, for each $\alpha \in \operatorname{Aut}_{\mathcal{F}}(S), \alpha([g])=[g]$. So by [AKO, Proposition I.8.4(a)], $\operatorname{trf}_{\mathcal{F}}([g])=[g]^{k} \neq 1$ where $k=\left|\operatorname{Out}_{\mathcal{F}}(S)\right|$ is odd. Thus $g \notin \mathfrak{f o c}(\mathcal{F})$ since $\operatorname{trf}_{\mathcal{F}}$ is well defined, so $\mathfrak{f o c}(\mathcal{F})<S, O^{2}(\mathcal{F}) \neq \mathcal{F}$ by Proposition 1.8, and $\mathcal{F}$ is not reduced.

The next lemma is an application of Lemma A.8, together with the transfer homomorphism for fusion systems (cf. [AKO, $\S$ I.8]). Recall that a finite group $G$ is metacyclic if it has a normal cyclic subgroup $H \unlhd G$ such that $G / H$ is also cyclic.

Lemma 2.4. Let $S$ be a finite 2-group, and let $\mathcal{F}$ be a saturated fusion system over $S$. Let $\mathcal{E}$ be the set of $\mathcal{F}$-essential subgroups of $S$.

(a) Assume $P \in \mathcal{E}$ is such that $\left[N_{S}(P), P\right]$ is cyclic. Then there are decompositions $P=P_{0} P_{1}$ and $\operatorname{Out}_{\mathcal{F}}(P)=\Gamma_{0} \times \Gamma_{1}$, where for $i=0,1,[P, P] \leq P_{i} \unlhd P, \Gamma_{i}$ sends $P_{i}$ to itself and acts trivially on $P_{1-i} /[P, P], \Gamma_{0}$ has odd order, and $\Gamma_{1} \cong \Sigma_{3}$. Either

(i) $P$ is abelian, $P_{1} \cong C_{2^{n}} \times C_{2^{n}}$ for some $n \geq 1, C_{P_{1}}\left(N_{S}(P)\right) \cong C_{2^{n}}$ and $\left[N_{S}(P), P\right] \cong C_{2^{n}}$ are both direct factors of $P_{1}$, and $P_{0} \cap P_{1}=1$; or

(ii) $P_{1} \cong Q_{8},\left[P_{0}, P_{1}\right]=1$, and $P_{0} \cap P_{1}=[P, P]=Z\left(P_{1}\right)$.

(b) If the image of $\operatorname{Aut}_{\mathcal{F}}(S)$ in $\operatorname{Aut}(S / Z(S))$ is a 2-group, then $\operatorname{Aut}_{\mathcal{F}}(S)=\Delta \times \operatorname{Inn}(S)$ for some (unique) subgroup $\Delta \leq \operatorname{Aut}_{\mathcal{F}}(S)$ of odd order.

(c) Assume $\mathcal{F}$ is reduced and the image of $\operatorname{Aut}_{\mathcal{F}}(S)$ in $\operatorname{Aut}(S / Z(S))$ is a 2-group. Assume also, for each $P \in \mathcal{E}$, that $\left[N_{S}(P), P\right]$ is cyclic and the factor $P_{0}$ of point (a) is contained in $Z(S)$. Then $\operatorname{Out}_{\mathcal{F}}(S)=1$, and $\Omega_{1}(Z(S)) \leq[S, S]$.

Proof. (a) By Lemma 1.5, $\left|\operatorname{Out}_{S}(P)\right|=\left|N_{S}(P) / P\right|=2$. If $P$ is abelian, then by Lemma A.8, $P$ and $\operatorname{Aut}_{\mathcal{F}}(P)$ have decompositions as described in (i). More precisely, Lemma A.8 says that $P_{1} \cong C_{2^{n}} \times C_{2^{n}}$ and $\left[N_{S}(P), P\right]=\left[N_{S}(P), P_{1}\right] \cong C_{2^{n}}$ for some $n \geq 1$, and hence that $\left[N_{S}(P), P\right]$ is a direct factor of $P_{1}$. Also, for $x \in N_{S}(P) \backslash P$, $C_{P_{1}}\left(N_{S}(P)\right)=C_{P_{1}}(x)$ is the kernel of the map $P_{1} \longrightarrow P_{1}$ which sends $g$ to $[x, g]$, so $P_{1} / C_{P_{1}}(x) \cong\left[x, P_{1}\right] \cong C_{2^{n}}$, and $C_{P_{1}}(x) \cong C_{2^{n}}$ is also a direct factor of $P_{1}$.

Assume $P$ is nonabelian. By Lemma A.2 (applied with $P_{0}=[P, P]$ ), the kernel of

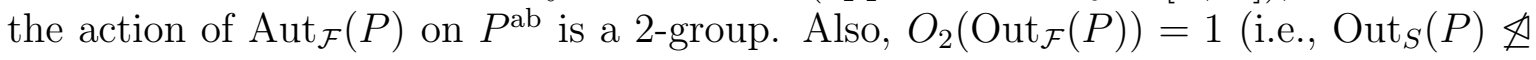


$\left.\operatorname{Out}_{\mathcal{F}}(P)\right)$, since $\operatorname{Out}_{\mathcal{F}}(P)$ has a strongly 2-embedded subgroup. Thus $\operatorname{Out}_{\mathcal{F}}(P)$ acts faithfully on $P^{\mathrm{ab}}$.

Set $P^{\prime}=[P, P] \neq 1$. By Lemma A.8, applied to the $\operatorname{Out}_{\mathcal{F}}(P)$-action on $P^{\mathrm{ab}}=P / P^{\prime}$, there are decompositions $P=P_{0} P_{1}$ and $\operatorname{Out}_{\mathcal{F}}(P)=\Gamma_{0} \times \Gamma_{1}$ such that $P_{i} \unlhd P$ is $\operatorname{Aut}_{\mathcal{F}}(P)$-invariant, $P_{0} \cap P_{1}=P^{\prime}, P_{1} / P^{\prime} \cong C_{2^{n}} \times C_{2^{n}}$ for some $n \geq 1$, $\Gamma_{0}$ has odd order and acts trivially on $P_{1} / P^{\prime}$, and $\Gamma_{1} \cong \Sigma_{3}$ acts trivially on $P_{0} / P^{\prime}$. Also (by the same lemma), $\left[N_{S}(P), P^{\mathrm{ab}}\right]=\left[N_{S}(P), P_{1} / P^{\prime}\right] \cong C_{2^{n}}$, so

$$
P_{1} /\left[N_{S}(P), P\right] \cong\left(P_{1} / P^{\prime}\right) /\left[N_{S}(P), P_{1} / P^{\prime}\right] \cong C_{2^{n}}
$$

Since $\left[N_{S}(P), P\right]$ is cyclic by assumption, $P_{1}$ is metacyclic.

Any $[\alpha] \in \Gamma_{1} \cong \Sigma_{3}$ of order 3 lifts to some $\alpha \in \operatorname{Aut}_{\mathcal{F}}(P)$, and upon replacing $\alpha$ by $\alpha^{k}$ for appropriate $k$, we can assume $\alpha$ has order 3 . If $P_{1}$ is abelian, then $P_{1} \cong C_{2^{n}} \times C_{2^{m}}$ where $m>n$ (since $P^{\prime} \neq 1$ ), which is impossible by Corollary A.3(a). So by Lemma A.7, $P_{1} \cong Q_{8}$. Hence $P_{1} / P^{\prime} \cong C_{2}^{2}$, and $P^{\prime}=\left[P_{1}, P_{1}\right]=Z\left(P_{1}\right) \cong C_{2}$.

For $x \in P_{0}$ and $y \in P_{1},[x, y]=\alpha([x, y])=[x, \alpha(y)]$ since $\left|P^{\prime}\right|=2$ and $\alpha$ acts trivially on $P_{0} / P^{\prime}$. Hence $\left[x, y^{-1} \alpha(y)\right]=1$, and $\left[P_{0}, P_{1}\right]=1$ since $\left[\alpha, P_{1}\right]=P_{1}$.

(b) Assume that the image of $\operatorname{Aut}_{\mathcal{F}}(S)$ in $\operatorname{Aut}(S / Z(S))$ is a 2-group. Then for each $\alpha \in \operatorname{Aut}_{\mathcal{F}}(S)$ of odd order, $\alpha$ induces the identity on $S / Z(S)$. So for each $g \in S$, there is $x \in Z(S)$ such that $\alpha(g)=x g$, and hence $\alpha c_{g} \alpha^{-1}=c_{\alpha(g)}=c_{g}$.

Thus each element of odd order in $\operatorname{Aut}_{\mathcal{F}}(S)$ commutes with $\operatorname{Inn}(S)$, so $\operatorname{Aut}_{\mathcal{F}}(S)=$ $\operatorname{Inn}(S) C_{\operatorname{Aut}_{\mathcal{F}}(S)}(\operatorname{Inn}(S))$. Since $\operatorname{Inn}(S)$ is 2-centric in $\operatorname{Aut}_{\mathcal{F}}(S), C_{\operatorname{Aut}_{\mathcal{F}}(S)}(\operatorname{Inn}(S))=$ $Z(\operatorname{Inn}(S)) \times \Delta$ where $\Delta$ has odd order (cf. [BLO1, Lemma A.4]). Thus $\operatorname{Aut}_{\mathcal{F}}(S)=$ $\operatorname{Inn}(S) \times \Delta$.

(c) Let $\Delta$ be as in (b), and set $Q=[\Delta, S] \leq Z(S)$. We first show that $Q \unlhd \mathcal{F}$.

Fix $P \in \mathcal{E}$, and let $P=P_{0} P_{1}$ and $\operatorname{Out}_{\mathcal{F}}(P)=\Gamma_{0} \times \Gamma_{1}$ be the decompositions of (a). For each $\delta \in \Delta, \delta(P)=P$ since $[\Delta, S]=Q \leq Z(S) \leq P$, so $\left.\delta\right|_{P} \in \operatorname{Aut}_{\mathcal{F}}(P)$. Also, $\left[\left.\delta\right|_{P}\right] \in N_{\operatorname{Out}_{\mathcal{F}}(P)}\left(\operatorname{Out}_{S}(P)\right)=\Gamma_{0} \times \operatorname{Out}_{S}(P)$ since $\delta\left(N_{S}(P)\right)=N_{S}(P)$, so $\left[\left.\delta\right|_{P}\right] \in \Gamma_{0}$ since it has odd order. Thus $[\delta, P] \leq P_{0}$. Hence

$$
Q=[\Delta, S]=[\Delta,[\Delta, S]] \leq[\Delta, P] \leq P_{0},
$$

where the second equality holds by $[\mathrm{G}$, Theorem 5.3.6].

Fix $\beta \in \operatorname{Aut}_{\mathcal{F}}(P)$ of odd order, and let $[\beta]$ be its class in $\operatorname{Out}_{\mathcal{F}}(P)$. If $[\beta] \in \Gamma_{0}$, then since $P_{0} \leq Z(S)$ by assumption, $\left.\beta\right|_{P_{0}}$ extends to an element of odd order in $\operatorname{Aut}_{\mathcal{F}}(S)$ by the extension axiom (i.e., since $P_{0}$ is fully centralized), and thus extends to an element of $\Delta$. So $\beta(Q)=Q$ in this case. If $[\beta] \in \Gamma_{1}$, then $\beta$ induces the identity on $P_{0} /[P, P]$ and on $[P, P]$ (since $|[P, P]| \leq 2$ ), and hence $\left.\beta\right|_{P_{0}}=\operatorname{Id}_{P_{0}}$ (and $\left.\beta\right|_{Q}=\operatorname{Id}_{Q}$ ) by Lemma A.2. Since $\operatorname{Aut}_{S}(P)$ acts trivially on $Z(S)$, this proves that all elements of $\operatorname{Aut}_{\mathcal{F}}(P)$ send $Q$ to itself.

Since this holds for each $P \in \mathcal{E}, Q \unlhd \mathcal{F}$ by Proposition 1.3. Hence $Q=[\Delta, S]=1$ since $\mathcal{F}$ is reduced, so $\operatorname{Out}_{\mathcal{F}}(S) \cong \Delta=1$, and $\Omega_{1}(Z(S)) \leq[S, S]$ by Proposition 2.3(a) (and since $\mathcal{F}$ is reduced).

The next proposition will be greatly generalized in Section 5, as a consequence of the results in Section 4 using amalgams.

Proposition 2.5. Let $S$ be any finite nonabelian 2-group such that $[S, S]$ is cyclic and $S$ has an abelian subgroup of index two. Then either $S \cong D_{2^{n}}(n \geq 3), S D_{2^{n}}(n \geq 4)$, or $C_{2^{n}} \nmid C_{2}(n \geq 2)$; or there is no reduced fusion system over $S$. 
Proof. Let $A<S$ be abelian of index two in $S$. By Lemma A.6(a), all elements in $(S / Z(S)) \backslash(A / Z(S))$ have order two, and $A / Z(S) \cong[S, S]$. Since $[S, S]$ is cyclic, $S / Z(S)$ is dihedral $\left(\right.$ or $\left.\cong C_{2}^{2}\right)$.

Assume $\mathcal{F}$ is a reduced fusion system over $S$, and let $\mathcal{E}$ be the set of $\mathcal{F}$-essential subgroups of $S$. We first show that the hypotheses of Lemma 2.4(c) hold. For each $P \in \mathcal{E},\left[N_{S}(P), P\right]$ is cyclic since $[S, S]$ is cyclic, and hence Lemma 2.4(a) applies to $P$. Let $P_{0}, P_{1} \unlhd P$ be as in that lemma; thus $P=P_{0} P_{1}$ and $P_{0} \cap P_{1}=[P, P]$.

If $P$ is abelian, it must be maximal abelian since it is centric. So either $P=A$, in which case $Z(S)=C_{P}(S)=C_{P}\left(N_{S}(P)\right)$ and $P / Z(S)=A / Z(S)$ is cyclic; or $P A=S$, in which case $P \cap A=Z(S)$, so $|P| Z(S) \mid=2, Z(S) \leq C_{P}\left(N_{S}(P)\right)<P$, and hence $Z(S)=C_{P}\left(N_{S}(P)\right)$. In either case, $Z(S)=C_{P}\left(N_{S}(P)\right) \geq P_{0}$ and $P / Z(S)$ is cyclic.

If $P$ is nonabelian, then by Lemma 2.4(a.ii), $P_{1} \cong Q_{8},[P, P]=Z\left(P_{1}\right)$, and $\left[P_{0}, P_{1}\right]=$ 1. In particular, $Z(S) \leq C_{P}\left(P_{1}\right)=P_{0}$. If $Z(S)<P_{0}$, then $P / Z(S)$ contains a subgroup isomorphic to $Q_{8}$ (if $\left.Z\left(P_{1}\right) \not \leq Z(S)\right)$ or $C_{2}^{3}$, both of which are impossible since $S / Z(S)$ is dihedral. Hence $Z(S)=P_{0}$.

Recall that $S / Z(S)$ is dihedral of order $\geq 4$. If $|S / Z(S)| \geq 8$, then $\operatorname{Aut}(S / Z(S))$ is a 2 -group by Corollary A.3(b). If $|S| Z(S) \mid=4$, then subgroups $A_{1}, A_{2}, A_{3}$ of index two in $S$ which contain $Z(S)$ are all abelian, they are the only proper subgroups centric in $S$, and hence the only subgroups which could be in $\mathcal{E}$. If $A_{i} \in \mathcal{E}$ (recall $\mathcal{E} \neq \varnothing$ by Lemma 2.2), then by Lemma 2.4(a.i), it contains a direct factor $A_{i 1} \cong C_{2^{m}} \times C_{2^{m}}$ (some $m \geq 1$ ), which in turn contains $C_{A_{i 1}}(S) \cong C_{2^{m}}$ and $[S, S]=\left[S, A_{i}\right] \cong C_{2^{m}}$ as direct factors. Thus $Z(S)=C_{A_{i}}(S) \cong A_{i 0} \times C_{2^{m}}$ and $[S, S]$ are both direct factors of $A_{i}$, and so $[S, S] \not \leq \operatorname{Fr}(Z(S))$. By Lemma A.6(d), there is no automorphism of $S$ which permutes the $A_{i}$ transitively, and thus the image of $\operatorname{Aut}_{\mathcal{F}}(S)$ in $\operatorname{Aut}(S / Z(S)) \cong \operatorname{Aut}\left(C_{2}^{2}\right)$ is a 2-group.

The hypotheses of Lemma 2.4(c) thus hold, and so $\Omega_{1}(Z(S)) \leq[S, S]$. Since $[S, S]$ is cyclic, this implies that $\left|\Omega_{1}(Z(S))\right|=2$, and hence that $Z(S)$ is cyclic.

If $|Z(S)|=2$, then $Z(S)=\Omega_{1}(Z(S)) \leq[S, S]$. So $S^{\mathrm{ab}} \cong(S / Z(S))^{\mathrm{ab}} \cong C_{2}^{2}$, which implies $S$ is dihedral, semidihedral, or quaternion (cf. [G, Theorem 5.4.5]). If $S \cong Q_{2^{n}}$, then by Lemma 2.4(a), for each $P \in \mathcal{E}, P \cong Q_{8}$ and $Z(P)=Z(S)$. Hence $Z(S) \unlhd \mathcal{F}$ by Proposition 1.3, which contradicts the assumption that $\mathcal{F}$ is reduced.

Now assume $|Z(S)|=2^{m}$ for $m \geq 2$; we will show that $S \cong C_{2^{m}} \succ C_{2}$. If $P \leq S$ is any nonabelian subgroup, then since $[S, S]$ is cyclic, $\Omega_{1}([S, S])=\Omega_{1}([P, P])$ is characteristic in $P$. So if all $\mathcal{F}$-essential subgroups are nonabelian, then $\Omega_{1}([S, S])$ is characteristic in each of them, and hence is normal in $\mathcal{F}$ by Proposition 1.3. Since this contradicts the assumption that $\mathcal{F}$ is reduced, there is an abelian subgroup $P \in \mathcal{E}$, and we already saw that this implies $P / Z(S)$ is cyclic. Since $Z(S)$ is also cyclic, $P$ has rank two, and $P \cong\left(C_{2^{n}}\right)^{2}$ by Lemma 2.4(a.i) (i.e., $P_{0}=1$ ). Then $n=m \geq 2$, since $Z(S)$ and $P / Z(S)$ are cyclic of order $2^{m}$ and $2^{2 n-m}$, respectively. Also, $P / Z(S) \cong C_{2^{m}}$ is normal in the dihedral group $S / Z(S)$, and hence $P \unlhd S$. So $[S: P]=2$ by Lemma 1.5 (and since $\operatorname{rk}(P)=2)$. Choose any $t \in S \backslash P$, fix $a \in P$ such that $a Z(S)$ generates $P / Z(S)$, and set $b=t a t^{-1}$. Then $a^{2^{m-1}} \notin Z(S)$ implies $1 \neq\left[t, a^{2^{m-1}}\right]=\left(b a^{-1}\right)^{2^{m-1}}$, so $\left|b a^{-1}\right|=2^{m}$, and $P=\langle a, b\rangle$. Also, $t b t^{-1}=a$ since $t^{2} \in P$, so $Z(S)=\langle a b\rangle$. Let $i$ be such that $t^{2}=(a b)^{i}$; then $\left(a^{-i} t\right)^{2}=1$, and this finishes the proof that $S \cong C_{2^{m}} \curlyvee C_{2}$.

In Section 5, as applications of our main theorems, we will generalize Proposition 2.5 by giving different (weaker) conditions on a 2-group each of which implies the conclusion 
of Proposition 2.5. For example, by Proposition 5.2(a,b), the same conclusion holds if $S$ has an abelian subgroup of index 2 or $[S, S]$ is cyclic.

\section{Two EXAMPLES}

For use in Section 4, we determine here the essential subgroups of the simple groups $\mathrm{PSU}_{3}(3)$ and $M_{12}$. At the same time, since reduced fusion systems over wreath products $C_{2^{n}} \curlyvee C_{2}$ play an important role in Section 5 (and in Proposition 2.5), we determine all reduced fusion systems over such groups.

We begin with the wreath products. Let $v_{2}(-)$ denote the 2-adic valuation: $v_{2}(n)=k$ if $2^{k} \mid n$ and $2^{k+1} \nmid n$.

Proposition 3.1. Assume $S=\langle a, b, t\rangle \cong C_{2^{m}} \chi C_{2}$ for some $m \geq 2$, where $A \stackrel{\text { def }}{=}\langle a, b\rangle \cong$ $C_{2^{m}} \times C_{2^{m}}, t^{2}=1$, and $t a t^{-1}=b$. Set

$$
Q=\left\langle a b, a^{2^{m-1}}, t\right\rangle \cong C_{2^{m}} \times_{C_{2}} D_{8} \cong C_{2^{m}} \times_{C_{2}} Q_{8} .
$$

(a) If $\mathcal{F}$ is a saturated fusion system over $S$, then the only subgroups of $S$ which could be $\mathcal{F}$-essential are $A$ and the subgroups $S$-conjugate to $Q$. If $O^{2}(\mathcal{F})=\mathcal{F}$, then all of these subgroups are $\mathcal{F}$-essential.

(b) Up to isomorphism, there is a unique saturated fusion system $\mathcal{F}$ over $S$ such that $O^{2}(\mathcal{F})=\mathcal{F}$. Also, $\mathcal{F}$ is reduced, and is isomorphic to the fusion system of $\operatorname{PSL}_{3}(q)$ for any prime power $q$ such that $v_{2}(q-1)=m$, and to the fusion system of $\operatorname{PSU}_{3}(q)$ for any prime power $q$ such that $v_{2}(q+1)=m$.

Proof. Let $\mathcal{F}$ be a saturated fusion system over $S$, and let $\mathcal{E}$ be the set of $\mathcal{F}$-essential subgroups of $S$. If $P \in \mathcal{E}$ and $\left|N_{S}(P) / P\right| \geq 4$, then $\operatorname{rk}(P / \operatorname{Fr}(P)) \geq 4$ by Lemma 1.5. Since $P \cap A$ is abelian of rank $\leq 2$ and has index $\leq 2$ in $P$, this is impossible. So $\left|N_{S}(P) / P\right|=2$.

Assume $P \neq A$. Since $P$ is centric in $S, Z(S)=\langle a b\rangle \leq P$. Also, $P \not \leq A$ since $P$ is centric, and $P \nsupseteq A$ since $P \notin\{A, S\}$. Thus $P \cap A=\left\langle a b, a^{2 i}\right\rangle$ for some $i$, and $P=\left\langle a b, a^{2 i}, a^{j} t\right\rangle$ for some $j$. If $a^{2 i}=1$, then $P$ is abelian with a cyclic subgroup $Z(S)$ of index two and order $\geq 4$, and $\operatorname{Aut}(P)$ is a 2-group by Corollary A.3(a). If $\left|a^{2 i}\right| \geq 4$, then $Z(P)=\langle a b\rangle=Z(S)$ is cyclic and $P / Z(P)$ is dihedral of order $2 \cdot\left|a^{2 i}\right| \geq 8$ (all elements in $(S / Z(S)) \backslash(A / Z(S))$ have order two by Lemma A.6(a)), so $\operatorname{Aut}(P)$ is a 2-group by Lemma A.2 and Corollary A.3(b). Thus $P$ can be essential only if $P=\left\langle a b, a^{2 i}, a^{j} t\right\rangle$ where $\left|a^{2 i}\right|=2$. If $j$ is odd, then $\left(a^{j} t\right)^{2}=(a b)^{j}$ generates $Z(S)$, $P^{\mathrm{ab}} \cong C_{2} \times C_{2^{m}}$, and $\operatorname{Aut}(P)$ is a 2 -group by Corollary A.3(a,c). This leaves only the possiblity $P=\left\langle a b, a^{2^{m-1}}, a^{j} t\right\rangle=a^{j / 2} Q a^{-j / 2}$ for even $j$. Thus $\mathcal{E} \subseteq\{A\} \cup \mathcal{Q}$, where $\mathcal{Q}$ denotes the $S$-conjugacy class of $Q$.

Now assume $O^{2}(\mathcal{F})=\mathcal{F}$. Then $\mathfrak{f} \mathfrak{c}(\mathcal{F})=S$ by Proposition 1.8. By Corollary A.3(a,c) (and since $S^{\mathrm{ab}} \cong C_{2^{m}} \times C_{2}$ ), $\operatorname{Aut}(S)$ is a 2-group, and so $\operatorname{Out}_{\mathcal{F}}(S)=1$. Hence $\mathfrak{f o c}(\mathcal{F}) \leq\langle[S, S], \mathcal{E}\rangle$. Since the images of $A$ and of $Q$ are both properly contained in $S^{\mathrm{ab}}, \mathcal{E}=\{A\} \cup \mathcal{Q}$. This proves (a).

Each of the three abelian subgroups of index two in $Q$ is isomorphic to $C_{2^{m}} \times C_{2}$ and contains exactly two elements of order 4 not in $Z(S)$. Hence $Q$ contains exactly six such elements, and they generate a subgroup $Q_{0}$ which is the unique subgroup of $Q$ isomorphic to $Q_{8}$. Since $Q=Q_{0} Z(S)$ and $Q_{0} \cap Z(S)=\left\langle(a b)^{2^{m-1}}\right\rangle$ (and $Z(S)=Z(Q)$ ), $\operatorname{Out}(Q)=\operatorname{Out}(Z(S)) \times \operatorname{Out}\left(Q_{0}\right)$, where $\operatorname{Out}\left(Q_{0}\right) \cong \Sigma_{3}$, and $\operatorname{Out}(Z(S))$ is a 2-group 
since $Z(S)$ is cyclic. Hence $\operatorname{Out}_{\mathcal{F}}(Q) \cong \Sigma_{3}$, and $\operatorname{Aut}_{\mathcal{F}}(Q)$ acts via the identity on $Z(S)$ and the full automorphism group of $Q_{0}$. Thus $\operatorname{Aut}_{\mathcal{F}}(Q)$ is uniquely determined.

By Lemma A.2, $\operatorname{Aut}(A) / O_{2}(\operatorname{Aut}(A)) \cong \operatorname{Aut}(A / \operatorname{Fr}(A)) \cong \Sigma_{3}$. Hence $\operatorname{Aut}_{\mathcal{F}}(A) \cong \Sigma_{3}$. Set $c=(a b)^{-1} \in Z(S)$, and let $\left\{a^{\prime}, b^{\prime}, c\right\}$ be its $\operatorname{Aut}_{\mathcal{F}}(A)$-orbit. Thus $a^{\prime}, b^{\prime}, c$ represent the three involutions in $A / \operatorname{Fr}(A)$, and any two of them generate $A$. Also, $a^{\prime} b^{\prime} c=1$ since $\operatorname{Aut}_{\mathcal{F}}(A)$ fixes $a^{\prime} b^{\prime} c$, and hence $c=\left(a^{\prime} b^{\prime}\right)^{-1}$.

Since $c \in Z(S)=C_{A}(t), c_{t}$ exchanges $a^{\prime}$ and $b^{\prime}$ and fixes $c$. We can thus assume the $a$ and $b$ were chosen so that $a=a^{\prime}$ and $b=b^{\prime}$. So up to an automorphism of $S$ (i.e., a relabelling of its generators), $\operatorname{Aut}_{\mathcal{F}}(A)$ is uniquely determined. Thus $\mathcal{F}$ is uniquely determined up to isomorphism by (a) and Theorem 1.2.

Now, $O^{2^{\prime}}(\mathcal{F})=\mathcal{F}$ since $\operatorname{Out}_{\mathcal{F}}(S)=1\left(\mathcal{F}\right.$ is generated by $O^{2^{\prime}}(\mathcal{F})$ and $\operatorname{Aut}_{\mathcal{F}}(S)$ by Theorem 1.2). If $P \unlhd \mathcal{F}$, then $P$ is contained in all $\mathcal{F}$-essential subgroups (cf. [AKO, Proposition I.4.5]), and hence is contained in their intersection $\left\langle a b, a^{2^{m-1}}\right\rangle$. Then $P \leq\langle a b\rangle$ since it is $\operatorname{Aut}_{\mathcal{F}}(Q)$-invariant, and so $P=1$ since it is $\operatorname{Aut}_{\mathcal{F}}(A)$-invariant. Thus $O_{2}(\mathcal{F})=1$, and $\mathcal{F}$ is reduced.

If $q$ is a prime power with $v_{2}(q-1)=m$, then $G L_{2}(q) \leq S L_{3}(q)$ and hence $P S L_{3}(q)$ have Sylow 2-subgroups isomorphic to $S$; while if $v_{2}(q+1)=m$, then $G U_{2}(q) \leq S U_{3}(q)$ and $\operatorname{PSU}_{3}(q)$ have Sylow 2-subgroups isomorphic to $S$ (cf. [CF, pp. 142-143]). Set $G=P S L_{3}(q)$ or $P S U_{3}(q)$, as appropriate, and identify $S \in \operatorname{Syl}_{2}(G)$. Since $G$ is simple, $\mathfrak{f o c}\left(\mathcal{F}_{S}(G)\right)=S \cap[G, G]=S$ by the focal subgroup theorem (cf. [G, Theorem 7.3.4]), and hence $\mathcal{F}_{S}(G) \cong \mathcal{F}$.

We now look at 2-groups of type $M_{12}$.

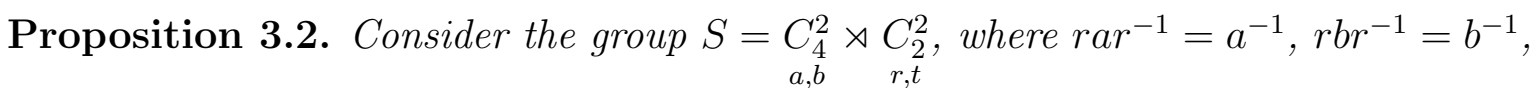
tat $^{-1}=b$, and $t b t^{-1}=a$. Set $A=\langle a, b\rangle \cong C_{4}^{2}, R=\langle A, r\rangle \cong C_{4}^{2} \rtimes C_{2}$, and

$$
Q=\left\langle a^{2}, a b, r, t\right\rangle=\left\langle a b^{-1}, a^{2} t\right\rangle \times_{\left\langle a^{2} b^{2}\right\rangle}\left\langle a b, a^{2} r t\right\rangle \cong Q_{8} \times_{C_{2}} Q_{8} .
$$

For any saturated fusion system $\mathcal{F}$ over $S$, the set of $\mathcal{F}$-essential subgroups is contained in $\{Q, R\}$, with equality if $\mathcal{F}$ is reduced.

Proof. Let $\mathcal{E}$ be the set of $\mathcal{F}$-essential subgroups of $S$. If $\mathcal{F}$ is reduced, then $|\mathcal{E}| \geq 2$ by Lemma 2.2. So it suffices to prove that $\mathcal{E} \subseteq\{R, Q\}$ for each saturated $\mathcal{F}$.

Fix $P \in \mathcal{E}$. Then $Z(S)=\left\langle a^{2} b^{2}\right\rangle \leq P$. Assume first that $\operatorname{Fr}(S)=\left\langle a^{2}, a b\right\rangle \not \leq P$, and fix $g \in \operatorname{Fr}(S) \backslash P$. Then $[g, P] \leq[g, S]=\left\langle a^{2} b^{2}\right\rangle$, so $\left|N_{S}(P) / P\right|=2$ by Lemma 1.5, and $a^{2} b^{2} \notin \operatorname{Fr}(P)$ by Lemma 1.4 (applied with $\Theta=1$ ). In particular, since $(a b)^{2}=\left(a b^{-1}\right)^{2}=a^{2} b^{2}$, neither $a b$ nor $a b^{-1}$ is in $P$. Hence $a^{2} \in P$, since $\left|N_{S}(P) / P\right|=2$. Also, $P \leq C_{S}\left(a^{2}\right)=\langle a, b, r\rangle$ since $a^{2} b^{2} \notin \operatorname{Fr}(P), N_{S}(P) \geq\langle a, b, r\rangle$ since $\langle a, b, r\rangle /\left\langle a^{2}, b^{2}\right\rangle$ is abelian and $P \geq\left\langle a^{2}, b^{2}\right\rangle$, and hence $[\langle a, b, r\rangle: P] \leq 2$. So up to $S$-conjugacy, $P=$ $\left\langle a, b^{2}, r\right\rangle$ or $\left\langle a, b^{2}, b r\right\rangle$ (recall $\left\langle a^{2}, a b\right\rangle \not \leq P$ ). In either case, $P \cong C_{2} \times D_{8}$, so $\operatorname{Aut}(P)$ is a 2-group by Lemma A.2 and since $P$ contains a unique subgroup isomorphic to $C_{2} \times C_{4}$, and hence $P \notin \mathcal{E}$.

Thus $P \geq \operatorname{Fr}(S)$, so $P \unlhd S$. If $[S: P] \geq 4$, then $|P| \leq 16, \operatorname{rk}(P / \operatorname{Fr}(P)) \geq 4$ by Lemma 1.5, and hence $P \cong C_{2}^{4}$. This is impossible since $P \geq \operatorname{Fr}(S) \cong C_{4} \times C_{2}$, and so $[S: P]=2$. If $P=\langle a, b, t\rangle$ or $\langle a, b, r t\rangle$, then $P /[P, P] \cong C_{4} \times C_{2}$, Aut $(P)$ is a 2-group by Corollary A.3(a,c), and hence $P \notin \mathcal{E}$. So $R=\langle a, b, r\rangle$ is the only (possible) subgroup in $\mathcal{E}$ which contains $A$. 
Now assume $P=P_{i j}=\left\langle a b, a^{2}, a^{i} r, a^{j} t\right\rangle$ for $i, j=0,1$ : these are the remaining four subgroups of index 2 in $S$. Let $Z_{2}(P) \unlhd P$ be the subgroup such that $Z_{2}(P) / Z(P)=$ $Z(P / Z(P))$. Then $Z(P)=\left\langle a^{2} b^{2}\right\rangle$ and $Z_{2}(P) \geq\left\langle a^{2}, a b\right\rangle$. If $(i, j) \neq(0,0)$, then the relations

$$
[r, a t]=a^{2}, \quad[a r, t]=a b^{-1}, \quad[a r, a t]=a^{-1} b^{-1}
$$

show that $Z_{2}(P)=\left\langle a^{2}, a b\right\rangle$. So $[a, P] \leq Z_{2}(P),\left[a, Z_{2}(P)\right]=1$, and $P \notin \mathcal{E}$ by Lemma 1.4. Thus $Q=P_{00}$ is the only possible subgroup in $\mathcal{E}$ which does not contain $A$.

With a little more work, one can show that the only reduced fusion systems over $S$ (as above) are those of $M_{12}$ and $G_{2}(3)$. But we leave that for a later paper.

\section{Detecting essential subgroups via Amalgams}

We are now ready to describe how theorems of Goldschmidt and Fan [Gd2, Fn] on amalgams can be used to get information about essential subgroups of index two in their normalizer for saturated fusion systems over 2-groups. Throughout the section, in the statements of lemmas and in the proofs of Theorems 4.5 and 4.6, we will refer repeatedly to the following set of hypotheses.

Assume $P_{1}, P_{2} \leq P \leq G_{1}, G_{2}$ are finite groups such that the following hold:

- $P$ is a 2-group, $\left[P: P_{1}\right]=\left[P: P_{2}\right]=2$, and $P=P_{1} P_{2}$.

- For $i=1,2, P_{i} \unlhd G_{i}, G_{i} / P_{i} \cong D_{2 p_{i}}$ for some odd prime $p_{i}$, and $C_{G_{i}}\left(P_{i}\right) \leq$ $P_{i}$.

Set $P_{12}=P_{1} \cap P_{2}$, and let $T \leq P_{12}$ be the largest subgroup which is normal in both $G_{1}$ and $G_{2}$.

Clearly, hypotheses $(*)$ imply that $\left[G_{i}: P\right]=p_{i}$ and $P_{i}=O_{2}\left(G_{i}\right)$ for $i=1,2$. In particular, in the terminology of Goldschmidt [Gd2], the triple $\left(G_{1}>P<G_{2}\right)$ is an amalgam of index $\left(p_{1}, p_{2}\right)$.

The following lemma helps to explain the motivation for these hypotheses.

Lemma 4.1. Fix a finite 2-group $S$ and a saturated fusion system $\mathcal{F}$ over $S$.

(a) Assume $P_{1}, P_{2} \leq S$ are distinct $\mathcal{F}$-essential subgroups of index two in $S$. Then there are groups $G_{1}>S<G_{2}$, and odd primes $p_{1}$ and $p_{2}$, such that $\operatorname{Out}_{G_{i}}\left(P_{i}\right) \leq$ $\operatorname{Out}_{\mathcal{F}}\left(P_{i}\right)$, and such that hypotheses $(*)$ hold with $P=S$.

(b) Let $P_{1} \leq S$ be an $\mathcal{F}$-essential subgroup which is not normal and has index two in its normalizer. Set $P=N_{S}\left(P_{1}\right)<S$, choose $x \in N_{S}(P) \backslash P$ such that $x^{2} \in P$, and set $P_{2}=x P_{1} x^{-1}$. Then there are groups $G_{1}>P<G_{2}$ and an odd prime $p$, such that $\operatorname{Out}_{\mathcal{F}}\left(P_{i}\right) \geq \operatorname{Out}_{G_{i}}\left(P_{i}\right)$ and hypotheses $(*)$ hold with $p_{1}=p_{2}=p$. Also, $x \in N_{S}(T)$, where $T$ is as defined in $(*)$, and there is an isomorphism $\beta: G_{1} \stackrel{\cong}{\longrightarrow} G_{2}$ such that $\left.\beta\right|_{P}=\left.c_{x}\right|_{P}$.

Proof. Assume the hypotheses of (a) or (b), where $P=S$ in (a). Since each $P_{i}$ is $\mathcal{F}$ essential (and $\left|N_{S}\left(P_{i}\right) / P_{i}\right|=2$ ), $\operatorname{Out}_{S}\left(P_{i}\right) \in \operatorname{Syl}_{2}\left(\operatorname{Out}_{\mathcal{F}}\left(P_{i}\right)\right)$ and is not the only Sylow 2-subgroup. Fix $1 \neq g \in \operatorname{Out}_{S}\left(P_{i}\right)$, and let $h \in \operatorname{Out}_{\mathcal{F}}\left(P_{i}\right)$ be any other involution. Then $\langle g, h\rangle$ is dihedral since it is generated by two involutions, and it has order $2 n$ for some odd integer $n>1$ since $4 \nmid\left|\operatorname{Out}_{\mathcal{F}}\left(P_{i}\right)\right|$. Upon choosing an appropriate subgroup 
$\Gamma_{i} \leq\langle g, h\rangle$, we can arrange that $\operatorname{Out}_{S}\left(P_{i}\right) \leq \Gamma_{i} \leq \operatorname{Out}_{\mathcal{F}}\left(P_{i}\right)$ and $\Gamma_{i} \cong D_{2 p_{i}}$ for some odd prime $p_{i}$.

Fix $i=1,2$, and set $\mathcal{F}_{i}=N_{\mathcal{F}}\left(P_{i}\right)$ (see Definition 1.1). This is a saturated fusion system over $N_{S}\left(P_{i}\right)=P$ (cf. [AKO, Theorem I.5.5]), and is constrained in the sense of [AKO, Definition I.4.8] since $P_{i} \unlhd \mathcal{F}_{i}$ and $C_{P}\left(P_{i}\right) \leq P_{i}$. By the model theorem in the form of [AKO, Theorem III.5.10(a)], there is a finite group $G_{i}^{*} \geq P$ such that $P_{i} \unlhd G_{i}^{*}$, $C_{G_{i}^{*}}\left(P_{i}\right) \leq P_{i}, P \in \operatorname{Syl}_{2}\left(G_{i}^{*}\right)$, and $\mathcal{F}_{P}\left(G_{i}^{*}\right)=\mathcal{F}_{i}$. In particular, $\operatorname{Out}_{G_{i}^{*}}\left(P_{i}\right)=\operatorname{Out}_{\mathcal{F}}\left(P_{i}\right)$. Let $G_{i} \leq G_{i}^{*}$ be the unique subgroup such that $P \leq G_{i}$ and $\operatorname{Out}_{G_{i}}\left(P_{i}\right)=\Gamma_{i}$. Then these groups satisfy hypotheses $(*)$.

In the situation of (b), we can assume that $\Gamma_{2}$ is chosen so that $\Gamma_{2}=\left[c_{x}\right] \Gamma_{1}\left[c_{x}\right]^{-1} \leq$ $\operatorname{Out}_{\mathcal{F}}\left(P_{2}\right)$. Choose $G_{1}$ as in (a), and then choose $G_{2}$ together with an isomorphism $\beta \in \operatorname{Iso}\left(G_{1}, G_{2}\right)$ such that $\left.\beta\right|_{P}=\left.c_{x}\right|_{P}$. Since $T$ is the unique largest subgroup of $P_{12}$ which is normal in $G_{1}$ and $G_{2}, x \in N_{S}(T)$ since $c_{x}$ exchanges $P_{1}$ and $P_{2}$ (recall $\left.x^{2} \in P=N_{S}\left(P_{1}\right)\right)$.

As usual, we say $T$ is centric in a group $X \geq T$ if $C_{X}(T) \leq T$. In general, our results using hypotheses $(*)$ split into separate cases, depending on whether or not $T$ is centric in $P$.

We say that a finite group $G$ is strictly $p$-constrained for a prime $p$ if $O_{p}(G)$ is centric in $G$. The question of whether one or both of the groups $G_{i} / T$ (under hypotheses (*)) is strictly 2-constrained plays an important role in Fan's classification of amalgams of type $\left(p_{1}, p_{2}\right)[\mathrm{Fn}]$.

Lemma 4.2. Assume hypotheses (*). Then the following hold.

(a) If $T$ is centric in $P$, then $T$ is also centric in $G_{1}$ and $G_{2}$.

(b) If $O_{p_{1}}\left(G_{1} / T\right) \neq 1$ or $O_{p_{2}}\left(G_{2} / T\right) \neq 1$, then $T$ is centric in $P$.

(c) For $i=1,2, G_{i} / T$ is strictly 2-constrained if and only if $O_{p_{i}}\left(G_{i} / T\right)=1$.

(d) Assume $T$ is centric in $P$, and let $S \geq P$ be any finite 2-group such that $N_{S}\left(P_{i}\right)=$ $P$ for $i=1,2$. Then $T$ is centric in $S$.

(e) Assume $T$ is centric in $P$, and let $S \geq P$ be as in (d). Set $\widehat{G}_{i}=\operatorname{Out}_{G_{i}}(T)$, $\widehat{S}=\operatorname{Out}_{S}(T)$, and $\widehat{G}=\left\langle\widehat{G}_{1}, \widehat{G}_{2}, \widehat{S}\right\rangle \leq \operatorname{Out}(T)$, and assume $\widehat{S} \in \operatorname{Syl}_{2}(\widehat{G})$. Then $\mathrm{O}_{2}(\widehat{G})=1$, and $\widehat{G}$ acts faithfully on $T / \operatorname{Fr}(T)$.

Proof. Let $S \geq P$ be any finite 2 -group as in (d); i.e., such that $N_{S}\left(P_{i}\right)=P$ for $i=1,2$. Set

$$
P^{0}=C_{P}(T), \quad G_{i}^{0}=C_{G_{i}}(T) \quad(i=1,2), \quad \text { and } \quad S^{0}=C_{S}(T) .
$$

Note that $P^{0} \unlhd P, G_{i}^{0} \unlhd G_{i}$, and $P^{0} \in \operatorname{Syl}_{2}\left(G_{i}^{0}\right)$ since $T$ is normal in $P$ and the $G_{i}$.

If $p_{i} \nmid\left|G_{i}^{0}\right|$, then $G_{i}^{0}$ is a 2-group, and $G_{i}^{0} \leq O_{2}\left(G_{i}\right)=P_{i}$ since $G_{i}^{0} \unlhd G_{i}$. If $P^{0}=G_{1}^{0}=$ $G_{2}^{0}$, then $P^{0} \leq P_{12}$ and is normal in $G_{1}$ and $G_{2}$, so $P^{0} \leq T$ by the maximality of $T$. To summarize:

$$
p_{i} \nmid\left|G_{i}^{0}\right| \Longrightarrow P^{0}=G_{i}^{0} \leq P_{i} \quad \text { and } \quad P^{0}=G_{1}^{0}=G_{2}^{0} \quad \Longrightarrow P^{0} \leq T .
$$

We next claim that when $i=1$ or 2 ,

$$
O_{p_{i}}\left(G_{i} / T\right) \neq 1 \quad \text { or } \quad P^{0} \leq T \quad \Longrightarrow \quad p_{i} \nmid\left|G_{i}^{0}\right| \text {. }
$$

To see this, assume $p_{i}|| G_{i}^{0} \mid$, and fix $g_{i} \in G_{i}^{0}$ of order $p_{i}$. Then $\left[g_{i}, T\right]=1$, while $\left[g_{i}, P_{i}\right] \neq 1$ by $(*)\left(C_{G_{i}}\left(P_{i}\right) \leq P_{i}\right)$. So by Lemma A.2, $g_{i}$ acts nontrivially on $P_{i} / T$; 
i.e., $\left[g_{i}, P_{i}\right] \not \leq T$. Thus $\left\langle T, g_{i}\right\rangle \unlhd G_{i}$, so $O_{p_{i}}\left(G_{i} / T\right)=1$. Also, $G_{i}^{0} T \unlhd G_{i}$ contains the normal closure of $\left\langle T, g_{i}\right\rangle$ in $G_{i}$, so $\left.G_{i}^{0} T\right\rangle\left\langle T, g_{i}\right\rangle, P^{0} T>T$, and thus $P^{0} \not \leq T$.

(a) If $T$ is centric in $P$ (i.e., $P^{0} \leq T$ ), then it is centric in the $G_{i}$ by (2) and (1).

(b) Let $H_{i} \unlhd G_{i}$ be the subgroup such that $H_{i} / T=O_{p_{i}}\left(G_{i} / T\right)$. If $H_{1} / T \neq 1$ and $H_{2} / T \neq 1$, then $P^{0} \leq T$ by (2) and (1) again. So assume $H_{1} / T \neq 1$ and $H_{2} / T=1$. By (2) and (1), $P^{0}=G_{1}^{0} \leq P_{1}$. Also, $P_{12} \unlhd G_{1}=H_{1} P$ since

$$
\left[P_{12}, H_{1}\right] \leq\left[P_{1}, H_{1}\right] \leq P_{1} \cap H_{1}=T \leq P_{12}
$$

(recall $P_{1} / T$ is a 2-group and $\left|H_{1} / T\right|=p_{1}$ ). Since $G_{1}^{0} \cap P=P^{0}=G_{2}^{0} \cap P$ and $G_{1}^{0} \leq P_{1}$,

$$
G_{2}^{0} \cap P_{2}=G_{1}^{0} \cap P_{2}=G_{1}^{0} \cap P_{12} \unlhd G_{1} .
$$

Also, $G_{2}^{0} \cap P_{2} \unlhd G_{2}$, so $G_{2}^{0} \cap P_{2} \leq T$ by the maximality of $T$, and the induced map $G_{2}^{0} T / T \longrightarrow G_{2} / P_{2} \cong D_{2 p_{2}}$ is injective. Then $O_{p_{2}}\left(G_{2}^{0} T / T\right)=1$ since $G_{2}^{0} T / T \unlhd G_{2} / T$ and $O_{p_{2}}\left(G_{2} / T\right)=1$, and hence $G_{2}^{0} T / T=1$ since it is isomorphic to a normal subgroup of $D_{2 p_{2}}$. Thus $P^{0} \leq G_{2}^{0} \leq T$, so $T$ is centric in $P$.

(c) By assumption, for $i=1,2, G_{i} / T$ is solvable of order $2^{n} p_{i}$ for some $n$. Hence the Fitting subgroup $F\left(G_{i} / T\right)=O_{2}\left(G_{i} / T\right) O_{p_{i}}\left(G_{i} / T\right)$ is always centric in $G_{i} / T$ (cf. [G, Theorem 6.1.3]). So $G_{i} / T$ is strictly 2 -constrained if and only if $O_{p_{i}}\left(G_{i} / T\right)=1$.

(d) Assume $T$ is centric in $P$. Then $S^{0} \unlhd N_{S}(T)$ and $S^{0} \cap P=P^{0} \leq T$. Since $N_{S}\left(P_{i}\right)=P$ by assumption, $N_{S^{0} P_{i}}\left(P_{i}\right)=P \cap S^{0} P_{i}=P_{i}$, so $S^{0} P_{i}=P_{i}$ by Lemma A.1. Thus $C_{S}(T)=S^{0} \leq P$, so $C_{S}(T)=C_{P}(T)$, and $T$ is centric in $S$ since it is centric in $P$.

(e) Assume $T$ is centric in $P$, and hence also centric in $G_{1}, G_{2}$, and $S$ by (a) and (d). Assume $\widehat{S} \in \operatorname{Syl}_{2}(\widehat{G})$, and set $Q=O_{2}(\widehat{G}) \leq \widehat{S}$ for short. For $i=1,2, Q \cap \widehat{G}_{i} \leq$ $\mathrm{O}_{2}\left(\widehat{G}_{i}\right)=\widehat{P}_{i}$, and hence

$$
N_{Q \widehat{P}_{i}}\left(\widehat{P}_{i}\right)=N_{\widehat{S}}\left(\widehat{P}_{i}\right) \cap Q \widehat{P}_{i}=\widehat{P} \cap Q \widehat{P}_{i}=(\widehat{P} \cap Q) \widehat{P}_{i}=\widehat{P}_{i} .
$$

By Lemma A.1, this implies $Q \widehat{P}_{i}=\widehat{P}_{i}$, and hence $Q \leq \widehat{P}_{i}$.

Thus $Q \leq \widehat{P}_{12}$. Hence $Q=\widehat{R}=\operatorname{Out}_{R}(T)$ for some unique $R \leq P_{12}$ such that $R \geq T$, and $R \unlhd G_{i}(i=1,2)$ since $Q=O_{2}(\widehat{G}) \unlhd \widehat{G}$. Thus $R=T$ by definition of $T$, and $Q=1$.

Since $\widehat{G} \leq \operatorname{Out}(T)$, the kernel of the induced $\widehat{G}$-action on $T / \operatorname{Fr}(T)$ is a 2-group by Lemma A.2, and is trivial since $O_{2}(\widehat{G})=1$. So the action is faithful.

The next lemma will be needed to handle the cases involving amalgams whose maximal normal subgroup is not centric. As usual, when $\mathcal{F}$ is a fusion system over a finite $p$-group $S$, a subgroup $P \leq S$ is strongly closed in $\mathcal{F}$ if no element of $P$ is $\mathcal{F}$-conjugate to an element of $S \backslash P$. For example, if $\mathcal{F}=\mathcal{F}_{S}(G)$ for a finite group $G$, and $H \unlhd G$, then $S \cap H$ is strongly closed in $\mathcal{F}$.

Lemma 4.3. Assume hypotheses (*), and also that $T$ is not centric in $P$. Set $\bar{P}=P / T$ and $\bar{G}_{i}=G_{i} / T$ for short, and let $\mathcal{F}^{*}$ be the smallest fusion system over $\bar{P}$ which contains $\mathcal{F}_{\bar{P}}\left(\bar{G}_{1}\right)$ and $\mathcal{F}_{\bar{P}}\left(\bar{G}_{2}\right)$. Assume

(a) $\mathcal{F}^{*}=\mathcal{F}_{\bar{P}}(\Gamma)$ for some finite perfect group $\Gamma$ for which $\bar{P} \in \operatorname{Syl}_{2}(\Gamma)$;

(b) no nontrivial proper subgroup of $\bar{P}$ is strongly closed in $\mathcal{F}^{*}$; 
(c) $\bar{G}_{i} / O^{2}\left(\bar{G}_{i}\right)$ is abelian for $i=1,2$; and

(d) $\left(\bar{P} \cap\left[\bar{G}_{1}, \bar{G}_{1}\right]\right) \cap\left(\bar{P} \cap\left[\bar{G}_{2}, \bar{G}_{2}\right]\right) \leq[\bar{P}, \bar{P}]$.

Then $C_{P}(T) \cdot T=P$, and $C_{G_{i}}(T) \cdot T=G_{i}$ for $i=1,2$. If, in addition, we define

$$
U_{i}=P \cap O^{2}\left(G_{i}\right), \quad U=U_{1} U_{2} \unlhd P, \quad \text { and } \quad Z=U \cap T,
$$

then $[U, T]=1, P=U T$, and $U \in \operatorname{Syl}_{2}(\widetilde{\Gamma})$ for some finite perfect group $\widetilde{\Gamma}$ such that $Z \leq Z(\widetilde{\Gamma})$ and $\widetilde{\Gamma} / Z \cong \Gamma$.

Proof. In general, for $X \leq G_{i}$ or $g \in G_{i}(i=1,2)$, we let $\bar{X}=X T / T$ or $\bar{g}=g T$ denote the image of $X$ or $g$, respectively, in $\bar{G}_{i}$. Write $P^{0}=C_{P}(T)$ and $G_{i}^{0}=C_{G_{i}}(T)$ for short. Then $G_{i}^{0} \unlhd G_{i}$ since $T \unlhd G_{i}$, and hence $\overline{G_{i}^{0}} \unlhd \bar{G}_{i}$. So $\overline{P^{0}}=\bar{P} \cap \overline{G_{i}^{0}}$ is strongly closed in $\mathcal{F}_{\bar{P}}\left(\bar{G}_{1}\right)$ and $\mathcal{F}_{\bar{P}}\left(\bar{G}_{2}\right)$, and hence is strongly closed in $\mathcal{F}^{*}=\left\langle\mathcal{F}_{\bar{P}}\left(\bar{G}_{1}\right), \mathcal{F}_{\bar{P}}\left(\bar{G}_{2}\right)\right\rangle$. Since $T$ is not centric in $P$ by assumption, $P^{0}=C_{P}(T) \not \leq T$, so $\overline{P^{0}} \neq 1$, and hence $\overline{P^{0}}=\bar{P}$ by (b). This in turn implies that $\overline{G_{i}^{0}} \geq \bar{P}$ for $i=1,2$, and hence $\overline{G_{i}^{0}}=\bar{G}_{i}$ since $\bar{G}_{i}$ is the normal closure of $\bar{P}$ (recall that $\bar{G}_{i} / \bar{P}_{i} \cong D_{2 p_{i}}$ ). We have now shown that

$$
C_{P}(T) \cdot T=P^{0} T=P \quad \text { and } \quad C_{G_{i}}(T) \cdot T=G_{i}^{0} T=G_{i} \quad(i=1,2) .
$$

In particular, $O^{2}\left(G_{i}\right)=O^{2}\left(G_{i}^{0}\right)$, and hence $U_{i}=P \cap O^{2}\left(G_{i}^{0}\right)$. Set

$$
U_{i}^{\bullet}=P \cap\left[G_{i}^{0}, G_{i}^{0}\right], \quad U^{\bullet}=U_{1}^{\bullet} U_{2}^{\bullet} \unlhd P^{0}, \quad \text { and } \quad Z^{\bullet}=U^{\bullet} \cap T .
$$

Then $U_{i} \leq U_{i}^{\bullet}$ (see Lemma A.4), and hence $U \leq U^{\bullet}$ and $Z \leq Z^{\bullet}$.

Now, $\bar{U}_{i}=\bar{P} \cap O^{2}\left(\bar{G}_{i}\right)=\bar{P} \cap\left[\bar{G}_{i}, \bar{G}_{i}\right]=\overline{U_{i}^{\bullet}}$, where the first and third equalities hold since $P \geq T$, and the second holds by (c) and Lemma A.4. Hence

$$
\bar{U}=\overline{U_{1}} \overline{U_{2}}=\left\langle\bar{P} \cap\left[\bar{G}_{1}, \bar{G}_{1}\right], \bar{P} \cap\left[\bar{G}_{2}, \bar{G}_{2}\right]\right\rangle=\mathfrak{f o c}\left(\mathcal{F}^{*}\right)=\mathfrak{f o c}\left(\mathcal{F}_{\bar{P}}(\Gamma)\right)=\bar{P},
$$

where the third and fifth equalities hold by the focal subgroup theorem (cf. [G, Theorem 7.3.4]) applied to $\bar{G}_{1}, \bar{G}_{2}$, and $\bar{\Gamma}$ (and since $\Gamma$ is perfect by (a)). Thus $U T=P$. So after taking intersections with $P^{0}=C_{P}(T)$, we get $U Z(T)=P^{0}$. Since $[U, Z(T)] \leq$ $\left[U^{\bullet}, T\right]=1$ by definition,

$$
[U, U]=\left[P^{0}, P^{0}\right] .
$$

Assume $u=u_{1} u_{2} \in T \cap U^{\bullet}=Z(T) \cap U^{\bullet}$, where $u_{i} \in U_{i}^{\bullet}$. Then $\bar{u}=1$ and $\bar{u}_{i} \in$ $\bar{P} \cap\left[\bar{G}_{i}, \bar{G}_{i}\right]$, so $\bar{u}_{1}=\bar{u}_{2}^{-1} \in[\bar{P}, \bar{P}]$ by $(\mathrm{d})$. Thus $u_{i} \in P^{0} \cap\left[P^{0} T, P^{0} T\right] T=\left[P^{0}, P^{0}\right] Z(T)$ (recall that $\left[P^{0}, T\right]=1$ ). Write $u_{i}=g_{i} t_{i}$ where $g_{i} \in\left[P^{0}, P^{0}\right] \leq U_{i}^{\bullet}$ and $t_{i} \in Z(T) \cap U_{i}^{\bullet}$. Since $Z(T) \cap U_{i}^{\bullet}=Z(T) \cap\left[G_{i}^{0}, G_{i}^{0}\right]=Z(T) \cap\left[P^{0}, P^{0}\right]$, the last equality by Proposition A.5, we see that $g_{1} g_{2}, t_{1}$, and $t_{2}$ all lie in $Z(T) \cap\left[P^{0}, P^{0}\right]$. This proves that

$$
Z^{\bullet}=T \cap U^{\bullet}=Z(T) \cap\left[P^{0}, P^{0}\right]=Z(T) \cap[U, U] \leq T \cap U=Z .
$$

Hence $Z=Z^{\bullet}$, since we already saw that $Z \leq Z^{\bullet}$.

Let $\tau \in H^{2}(\bar{P} ; Z(T))$ and $\tau_{i} \in H^{2}\left(\bar{G}_{i} ; Z(T)\right)$ be the classes of the central extensions $P^{0}$ and $G_{i}^{0}$, respectively. Thus $\tau=\left.\tau_{i}\right|_{\bar{P}}$, so $\tau$ is stable with respect to fusion in $\bar{G}_{i}$, and hence is stable with respect to the fusion system $\mathcal{F}^{*}$. Since $\mathcal{F}^{*}=\mathcal{F}_{\bar{P}}(\Gamma)$ by $(\mathrm{a}), \tau$ is the restriction of a unique element $\tau_{\Gamma} \in H^{2}(\Gamma ; Z(T))$, where $\Gamma$ acts trivially on $Z(T)$ (cf. [CE, Theorem XII.10.1]). Let $\widehat{\Gamma}$ be the corresponding central extension of $Z(T)$ by $\Gamma$. Thus $P^{0} \in \operatorname{Syl}_{2}(\widehat{\Gamma})$. 
Set $\widetilde{\Gamma}=[\widehat{\Gamma}, \widehat{\Gamma}]$. Since $\widehat{\Gamma} / Z(T) \cong \Gamma$ is perfect, $\widehat{\Gamma}=\widetilde{\Gamma} \cdot Z(T)$ where $[\widetilde{\Gamma}, Z(T)]=1$, so $\widetilde{\Gamma}=[\widehat{\Gamma}, \widehat{\Gamma}]=[\widetilde{\Gamma}, \widetilde{\Gamma}]$. Thus $\widetilde{\Gamma}$ is perfect. Since $P^{0} \in \operatorname{Syl}_{2}(\widehat{\Gamma})$, we have

$$
Z=Z^{\bullet}=Z(T) \cap\left[P^{0}, P^{0}\right]=Z(T) \cap[\widehat{\Gamma}, \widehat{\Gamma}]=Z(T) \cap \widetilde{\Gamma},
$$

the third equality by Proposition A.5. Recall that $P^{0}=U Z(T)$. Hence $P^{0} / U \cong$ $Z(T) / Z \cong \Gamma / \widetilde{\Gamma}$, and so $U \in \operatorname{Syl}_{2}(\widetilde{\Gamma})$.

We recall the terminology which will be used in the statements of our main theorems. By an amalgam is meant here a triple of groups $\mathcal{G}=\left(G_{1}>H<G_{2}\right)$. A (proper) completion of $\mathcal{G}$ is a group $G$, together with injections $\rho_{i}: G_{i} \longrightarrow G$, such that $\left.\rho_{1}\right|_{H}=$ $\left.\rho_{2}\right|_{H}$ and $G=\left\langle\rho_{1}\left(G_{1}\right), \rho_{2}\left(G_{2}\right)\right\rangle$. The universal completion is the amalgamated free product $G_{1} * G_{2}$. When $H$ has prime index in $G_{1}$ and in $G_{2}$, then the amalgam $\mathcal{G}$ is primitive if no nontrivial subgroup of $H$ is normal in $G_{1}$ and in $G_{2}$. An isomorphism of amalgams from $\left(G_{1}>H<G_{2}\right)$ to $\left(G_{1}^{*}>H^{*}<G_{2}^{*}\right)$ is a triple of isomorphisms $\alpha: H \stackrel{\cong}{\longrightarrow} H^{*}$ and $\beta_{i}: G_{i} \stackrel{\cong}{\longrightarrow} G_{i}^{*}$ such that $\left.\beta_{1}\right|_{H}=\alpha=\left.\beta_{2}\right|_{H}$.

Lemma 4.4. For each $n=1, \ldots, 6$, let $\bar{G}_{1}=\bar{G}_{1}^{(n)}, \bar{G}_{2}=\bar{G}_{2}^{(n)}$, and $\Gamma=\Gamma^{(n)}$ be the groups listed in case $(n)$ of Table 4.1. Then there is a primitive amalgam $\left(\bar{G}_{1}>\bar{S}<\bar{G}_{2}\right)$ with completion $\Gamma$, where $\bar{S}=\bar{S}^{(n)} \in \operatorname{Syl}_{2}\left(\bar{G}_{i}\right)$ and $\left[\bar{G}_{i}: \bar{S}\right]=3$ for $i=1,2$, and this amalgam is uniquely determined up to isomorphism. Set $\mathcal{F}^{(n)}=$ $\left\langle\mathcal{F}_{\bar{S}^{(n)}}\left(\bar{G}_{1}^{(n)}\right), \mathcal{F}_{\bar{S}^{(n)}}\left(\bar{G}_{2}^{(n)}\right)\right\rangle$.

(a) For each $n=1,3,5, \Gamma^{(n)}$ can be identified with a subgroup of index two in $\Gamma^{(n+1)}$ in such a way that the amalgam $\left(\bar{G}_{1}^{(n)}>\bar{S}^{(n)}<\bar{G}_{2}^{(n)}\right)$ is contained in $\left(\bar{G}_{1}^{(n+1)}>\right.$ $\left.\bar{S}^{(n+1)}<\bar{G}_{2}^{(n+1)}\right)$ with index two, and the normal closure of $\bar{S}^{(n)}$ in $\bar{G}_{i}^{(n+1)}$ equals $\bar{G}_{i}^{(n)}$ for $i=1,2$.

(b) For each $1 \leq n \leq 6$, if $Q \leq \bar{S}^{(n)}$ is strongly closed in $\mathcal{F}^{(n)}$, then either $Q=1$ or $Q=\bar{S}^{(n)}$, or $n$ is even and $Q=\bar{S}^{(n-1)}$.

(c) For $n=1,3,5, \mathcal{F}^{(n)}=\mathcal{F}_{\bar{S}^{(n)}}\left(\Gamma^{(n)}\right)$. (This also holds when $n=2,4,6$, but we will not need that.)

Proof. These are the amalgams denoted $G_{i}$ and $G_{i}^{1}$ for $i=3,4,5$ in [Gd2, Table 1]. In all but the first case, Goldschmidt's choice of completion is the same as the one listed here in Table 4.1. (Note that $\operatorname{Aut}\left(U_{3}(3)\right) \cong G_{2}(2)$.) In case (1), Goldschmidt lists $L_{3}(2)$ as a completion, but $A_{6}$ is easily seen to be a completion for the same amalgam. The uniqueness of the amalgams (for given $\bar{G}_{i}$ and $\Gamma$ ) follows from the classification in $[\mathrm{Gd} 2$, Theorem A].

Point (a) follows from Goldschmidt's construction of the amalgams [Gd2, 3.5, 3.7, 3.8], and also by a direct inspection of the groups in question.

Point (b) can be checked case-by-case. When $n$ is even, it follows from point (a), point (b) for $n-1$, and the observation that no central subgroup of order two in $\bar{S}^{(n)}$ is strongly closed. So assume $n$ is odd. Since $Z\left(\bar{S}^{(n)}\right)$ is cyclic in all three cases $(n=1,3,5)$, each nontrivial normal subgroup of $\bar{S}=\bar{S}^{(n)}$ contains $\Omega_{1}(Z(\bar{S})$ ).

Consider, for example, the case $n=5$, where $\bar{S}=\langle a, b, r, t\rangle$ in the notation of Proposition 3.2. Set $A=\langle a, b\rangle \cong C_{4}^{2}, A_{0}=\Omega_{1}(A)=\left\langle a^{2}, b^{2}\right\rangle, \bar{P}_{1}=O_{2}\left(\bar{G}_{1}\right)=$

\footnotetext{
${ }^{1}$ The groups $\bar{G}_{1}$ in cases (4) and (5) are not isomorphic. See [Gd2, Table 1] for more details.
} 


\begin{tabular}{|c|c|c|c|c|c|}
\hline & $\left(\bar{G}_{1}, \bar{G}_{2}\right) \cong$ & $\Gamma$ & $U \cong$ & $|Z|$ & {$[S: U T]$} \\
\hline \multirow{2}{*}{ (1) } & \multirow{2}{*}{$\left(\Sigma_{4}, \Sigma_{4}\right)$} & \multirow{2}{*}{$A_{6}$} & $D_{8}$ & 1 & 1 \\
\hline & & & $Q_{16}$ & 2 & 1 \\
\hline \multirow{2}{*}{ (2) } & \multirow{2}{*}{$\left(C_{2} \times \Sigma_{4}, C_{2} \times \Sigma_{4}\right)$} & \multirow{2}{*}{$\Sigma_{6}$} & $D_{8}$ & 1 & 2 \\
\hline & & & $Q_{16}$ & 2 & 2 \\
\hline (3) & $\left(\left(Q_{8} \times_{C_{2}} C_{4}\right) \cdot \Sigma_{3}, C_{4}^{2} \rtimes \Sigma_{3}\right)$ & $U_{3}(3)$ & $C_{4} \prec C_{2}$ & 1 & 1 \\
\hline (4) & $\left(\left(Q_{8} \times_{C_{2}} Q_{8}\right) \rtimes \Sigma_{3}, C_{4}^{2} \rtimes D_{12}\right)^{1}$ & $\operatorname{Aut}\left(U_{3}(3)\right)$ & $C_{4} \prec C_{2}$ & 1 & 2 \\
\hline \multirow{2}{*}{ (5) } & \multirow{2}{*}{$\left(\left(Q_{8} \times_{C_{2}} Q_{8}\right) \rtimes \Sigma_{3}, C_{4}^{2} \rtimes D_{12}\right)^{1}$} & \multirow{2}{*}{$M_{12}$} & $\operatorname{Syl}_{2}\left(M_{12}\right)$ & 1 & 1 \\
\hline & & & $\operatorname{Syl}_{2}\left(2 M_{12}\right)$ & 2 & 1 \\
\hline \multirow{2}{*}{ (6) } & \multirow{2}{*}{$\begin{array}{r}\left(\left(Q_{8} \times_{C_{2}} Q_{8}\right) \cdot D_{12}\right. \\
\left.\quad C_{4}^{2} \rtimes\left(\left(C_{2}^{2} \times C_{3}\right) \rtimes C_{2}\right)\right)\end{array}$} & \multirow{2}{*}{$\operatorname{Aut}\left(M_{12}\right)$} & $\operatorname{Syl}_{2}\left(M_{12}\right)$ & 1 & 2 \\
\hline & & & $\operatorname{Syl}_{2}\left(2 M_{12}\right)$ & 2 & 2 \\
\hline
\end{tabular}

TABLE 4.1

$\left\langle a^{2}, a b, r, t\right\rangle \cong Q_{8} \times_{C_{2}} Q_{8}$, and $\bar{P}_{2}=O_{2}\left(\bar{G}_{2}\right)=\langle A, r\rangle \cong C_{4}^{2} \rtimes C_{2}$. If $1 \neq P_{*} \leq \bar{S}$ is strongly closed, then $P_{*} \geq Z(\bar{S})=\left\langle a^{2} b^{2}\right\rangle$ and $P_{*} \cap \bar{P}_{2} \unlhd \bar{G}_{2}$ imply $P_{*} \geq A_{0}$ (since $A_{0}=$ $\left\langle a^{2}, b^{2}\right\rangle$ is the normal closure in $\bar{G}_{2}$ of $\left.\left\langle a^{2} b^{2}\right\rangle\right)$. If $P_{*} / A_{0} \leq Z\left(\bar{S} / A_{0}\right)=\left\langle A_{0}, a b, r\right\rangle / A_{0}$, then $P_{*} \leq \bar{P}_{1} \cap \bar{P}_{2}$ and $P_{*} \unlhd \bar{G}_{i}$ for $i=1,2$, which contradicts the assumption that the amalgam is primitive. Thus $P_{*} / A_{0} \not \leq Z\left(\bar{S} / A_{0}\right)$ and $P_{*} \unlhd \bar{S}$. Every normal subgroup of $\bar{S} / A_{0} \cong D_{8} \times C_{2}$ is either contained in its center or contains its commutator subgroup, so $P_{*} \geq[\bar{S}, \bar{S}]=\left\langle A_{0}, a b\right\rangle$. But then $P_{*} \geq A$ since $P_{*} \cap \bar{P}_{2} \unlhd \bar{G}_{2}$, and so $P_{*}=\bar{S}$ since $P_{*} \cap \bar{P}_{1} \unlhd \bar{G}_{1}$ (and by [A2, Lemma $\left.5.3(2)\right]$ ).

Point (c) is clear for $n=1$. When $n=3$ or 5 , then $\mathcal{F}^{(n)} \subseteq \mathcal{F}_{\bar{S}}(\Gamma)$ since $\Gamma$ is a proper completion of $\left(\bar{G}_{1}>\bar{S}<\bar{G}_{2}\right)$, and $\bar{P}_{i} \stackrel{\text { def }}{=} O_{2}\left(\bar{G}_{i}\right)$ for $i=1,2$ are the only (possible) $\mathcal{F}_{\bar{S}}(\Gamma)$-essential subgroups of $\Gamma$ by Propositions 3.1 or 3.2 , respectively. So to show that $\mathcal{F}^{(n)}=\mathcal{F}_{\bar{S}}(\Gamma)$, it suffices to show that $\operatorname{Out}_{\bar{G}_{i}}\left(\bar{P}_{i}\right) \cong \operatorname{Out}_{\Gamma}\left(\bar{P}_{i}\right)$ for $i=1,2$. When $n=5$ and $i=1$, this is shown in [A2, Lemma 5.3(2)], and in all of the other cases, it follows since $\operatorname{Aut}\left(\bar{P}_{i}\right) / O_{2}\left(\operatorname{Aut}\left(\bar{P}_{i}\right)\right) \cong \Sigma_{3}$ by Lemma A.2.

Theorem 4.5. Let $\mathcal{F}$ be a saturated fusion system over a finite 2-group $S$, with distinct $\mathcal{F}$-essential subgroups $P_{1}$ and $P_{2}$ of index two in $S$. Then there are finite groups $G_{1}>$ $S<G_{2}$ such that for $i=1,2, P_{i} \unlhd G_{i}, G_{i} / P_{i} \cong \operatorname{Out}_{G_{i}}\left(P_{i}\right) \cong D_{2 p_{i}}$ for some odd prime $p_{i}$, and $\operatorname{Aut}_{G_{i}}\left(P_{i}\right) \leq \operatorname{Aut}_{\mathcal{F}}\left(P_{i}\right)$. Let $T \leq P_{1} \cap P_{2}$ be the largest subgroup normal in both $G_{1}$ and $G_{2}$. Then these groups satisfy hypotheses $(*)$ with $P=S$.

If $T$ is not centric in $S$, then $p_{1}=p_{2}=3$, and $\left(G_{1} / T>S / T<G_{2} / T\right)$ is one of the amalgams listed in Table 4.1. Set

$$
U_{i}=S \cap O^{2}\left(G_{i}\right) \quad(i=1,2), \quad U=U_{1} U_{2}, \quad \text { and } \quad Z=U \cap T .
$$

Then $U \unlhd S,[U, T]=1$; and $\bar{G}_{i}=G_{i} / T, U, Z$, and $[S: U T]$ are as listed in Table 4.1. 
Proof. Set $P=S$. By Lemma 4.1(a), there are groups $G_{1}>P<G_{2}$ as described such that the $P_{i}<P<G_{i}$ satisfy hypotheses $(*)$. Let $T$ be as in $(*)$, and set $\bar{G}_{i}=G_{i} / T$ and $\bar{S}=S / T$.

Assume $T$ is not centric in $S$. By Lemma $4.2(\mathrm{~b}, \mathrm{c}), O_{p_{i}}\left(\bar{G}_{i}\right)=1$, and $\bar{G}_{i}$ is strictly 2constrained, for $i=1,2$. So by a theorem of Fan [Fn, Theorem 1], either $\left(\bar{G}_{1}>\bar{S}<\bar{G}_{2}\right)$ is one of the amalgams listed by Goldschmidt in [Gd2, Table 1], or it is the ${ }^{2} F_{4}(2)^{\prime}$ or ${ }^{2} F_{4}(2)$-amalgam (points (2) and (3) in [Fn, Theorem 1]). It cannot be either of the last two, since that would require that $G_{i} / O_{2}\left(G_{i}\right) \cong S z(2) \cong C_{5} \rtimes C_{4}$ for $i=1$ or 2 (see, e.g., [Wi, Theorem 1], or the discussion in [Car, § 8.5] of the Levi decomposition of parabolic subgroups of groups of Lie type). Hence it is one of the six amalgams listed in Table 4.1, since the others listed by Goldschmidt involve groups which are not strictly 2-constrained.

Assume we are in case $(n)$ in Table 4.1. When $n$ is odd, we apply Lemma 4.3. Conditions (a) and (b) in the lemma were shown in Lemma 4.4(c,b), respectively, and (c) and (d) are easily checked case-by-case. So by that lemma, $[U, T]=1, U T=S$, and $U \in \operatorname{Syl}_{2}(\widetilde{\Gamma})$ for some finite perfect group $\widetilde{\Gamma}$ such that $Z \leq Z(\widetilde{\Gamma})$ and $\widetilde{\Gamma} / Z \cong \Gamma$. Here, $\Gamma \cong A_{6}, U_{3}(3)$, or $M_{12}$, when $n=1,3$, or 5 . These groups have Schur multiplier $C_{6}$, 1 , and $C_{2}$, respectively (see [A1, (33.15)], [Gr, Theorem 2], and [Mz]). Hence $|Z| \leq 2$, with equality possible only when $n=1$ or 5 .

When $n$ is even, then by Lemma 4.4(a), there are subgroups $\widetilde{G}_{i}<G_{i}$ and $\widetilde{S}<S$ of index two, all containing $T$, such that $\widetilde{S}=S \cap \widetilde{G}_{i}$, and such that $\left(\widetilde{G}_{1} / T>\widetilde{S} / T<\widetilde{G}_{2} / T\right)$ is an amalgam of type $(n-1)$. Hence $U_{i}=S \cap O^{2}\left(G_{i}\right)=\widetilde{S} \cap O^{2}\left(\widetilde{G}_{i}\right)$, and so $U=U_{1} U_{2}$ plays the same role for the new amalgam as for the original one. Also, (*) holds for $\left(\widetilde{G}_{1}>\widetilde{S}<\widetilde{G}_{2}\right)$, and $T$ is the largest subgroup normal in $\widetilde{G}_{1}$ and $\widetilde{G}_{2}$ since the quotient amalgam is primitive. If $T$ were centric in $\widetilde{S}$, then it would be centric in the $\widetilde{G}_{i}$ by Lemma 4.2(a), and since $T$ is not centric in $S, T \cdot C_{S}(T)=T \cdot C_{G_{i}}(T)>T$ would be a strictly larger subgroup normal in the $G_{i}$. Since this contradicts the choice of $T$, we conclude that $T$ is not centric in $\widetilde{S}$, and hence that the result follows by the argument in the last paragraph applied to $\left(\widetilde{G}_{1}>\widetilde{S}<\widetilde{G}_{2}\right)$.

Theorem 4.5 will be used when looking for pairs of essential subgroups of index two in $S$. We next turn to the problem of identifying essential subgroups which have index two in their normalizer but are not normal. The idea is to apply the classification of amalgams by Goldschmidt and Fan to a pair of essential subgroups which are conjugate in $S$, and have index two in their common normalizer.

Theorem 4.6. Let $\mathcal{F}$ be a saturated fusion system over a finite 2-group $S$. Let $P_{1} \leq S$ be an $\mathcal{F}$-essential subgroup which is not normal in $S$, and such that $\left|N_{S}\left(P_{1}\right) / P_{1}\right|=2$. Set $P=N_{S}\left(P_{1}\right)<S$. Choose $x \in N_{S}(P) \backslash P$ such that $x^{2} \in P$, and set $P_{2}=x P_{1} x^{-1}$. Then there are finite groups $G_{1}>P<G_{2}$, an odd prime $p$, and an isomorphism $\beta: G_{1} \stackrel{\cong}{\longrightarrow} G_{2}$ such that $\left.\beta\right|_{P}=c_{x} \in \operatorname{Aut}_{S}(P)$, and for $i=1,2, \operatorname{Out}_{G_{i}}\left(P_{i}\right) \leq \operatorname{Out}_{\mathcal{F}}\left(P_{i}\right)$, $P_{i} \unlhd G_{i}$, and $\operatorname{Out}_{G_{i}}\left(P_{i}\right) \cong G_{i} / P_{i} \cong D_{2 p}$. Let $T \leq P_{1} \cap P_{2}$ be the largest subgroup which is normal in $G_{1}$ and in $G_{2}$.

(a) If $T$ is not centric in $S$, then $p=3$. Set $U_{i}=P \cap O^{2}\left(G_{i}\right) \unlhd P, U=U_{1} U_{2}$, and $Z=T \cap U$. Set $W=\operatorname{Fr}(U), S_{*}=N_{S}(W)$, and let $\Delta$ be the normal closure of $U$ in $S_{*}$. Then the following hold. 
(i) $[T, U]=1$; and either $U_{i} \cong C_{2}^{2}, U \cong D_{8}$, and $Z=1$; or $U_{i} \cong Q_{8}, U \cong Q_{16}$, and $Z=Z(U)$.

(ii) There is a subgroup $T^{\bullet} \unlhd P$ such that $T \leq T^{\bullet}<P_{1} \cap P_{2},\left[T^{\bullet}: T\right] \leq 2$;

(ii.1) $P_{i}=T^{\bullet} U_{i}$ and $T^{\bullet} \cap U=T \cap U=Z$; and

(ii.2) $\left[S_{*}: T^{\bullet} \Delta\right]=2, C_{S_{*}}\left(U_{1}\right)=T^{\bullet} U_{1}$ if $U \cong D_{8}, C_{S_{*}}\left(U_{1}\right)=T^{\bullet}$ if $U \cong Q_{16}$.

Also, $G_{i} / T,|Z|, U_{i}, U, \Delta,\left[T^{\bullet}: T\right]$, and $\left[T^{\bullet}, U\right]$ are as described in Table 4.2.

(b) If $T$ is centric in $P$ or (equivalently) centric in $S$, then $[S, S]$ is nonabelian, $\mathrm{O}_{2}\left(\operatorname{Out}_{\mathcal{F}}(T)\right)=1$, and $\operatorname{Out}_{\mathcal{F}}(T)$ acts faithfully on $T / \operatorname{Fr}(T)$.

(c) In the situation of $(a)$, if $[S, S]$ is abelian, or if $T^{\bullet} / Z$ is abelian, or more generally if $T^{\bullet}$ contains no quaternion subgroup of order 16 and $T^{\bullet} / Z$ contains no dihedral subgroup of order 8 , then $S_{*}=S$.

\begin{tabular}{|c|c||c|c|c|c|c|}
\hline$G_{i} / T \cong$ & $|Z|$ & $U_{i} \cong$ & $U \cong$ & $\Delta \cong$ & $\left|T^{\bullet} / T\right|$ & {$\left[T^{\bullet}, U\right]$} \\
\hline \hline$\Sigma_{4}$ & 1 & $C_{2}^{2}$ & $D_{8}$ & $D_{2^{n}}, n \geq 3$ & 1 & 1 \\
\hline$\Sigma_{4}$ & 2 & $Q_{8}$ & $Q_{16}$ & $Q_{2^{n}}, n \geq 4$ & 1 & 1 \\
\hline$\Sigma_{4} \times C_{2}$ & 1 & $C_{2}^{2}$ & $D_{8}$ & $D_{2^{n}}, n \geq 3$ & 2 & 1 \\
\hline$\Sigma_{4} \times C_{2}$ & 2 & $Q_{8}$ & $Q_{16}$ & $Q_{2^{n}}, n \geq 4$ & 2 & $Z$ \\
\hline
\end{tabular}

TABLE 4.2

Proof. By Lemma 4.1(b), there are finite groups $G_{1}>P<G_{2}$ such that $P_{i} \unlhd G_{i}$, $G_{i} / P_{i} \cong D_{2 p}$ for some odd prime $p, \operatorname{Out}_{S}\left(P_{i}\right) \leq \operatorname{Out}_{G_{i}}\left(P_{i}\right) \leq \operatorname{Out}_{\mathcal{F}}\left(P_{i}\right)$, and $\left.\beta\right|_{P}=\left.c_{x}\right|_{P}$ for some $\beta \in \operatorname{Iso}\left(G_{1}, G_{2}\right)$. Thus $(*)$ holds with $p_{1}=p_{2}=p$. Also, $x \in N_{S}(T)$, where $T \leq P_{1} \cap P_{2}$ is the largest subgroup normal in $G_{1}$ and $G_{2}$.

(a) If $T$ is not centric in $S$, then it is not centric in $P$ by Lemma $4.2(\mathrm{~d})$. Hence the $G_{i} / T$ are strictly 2-constrained by Lemma 4.2(b,c). By [Fn, Theorem 1], they are among the groups listed in [Gd2, Table 1], and hence are isomorphic to $\Sigma_{4}$ or $C_{2} \times \Sigma_{4}$ (and $p=3$ ).

Case 1: $\boldsymbol{G}_{\boldsymbol{i}} / \boldsymbol{T} \cong \boldsymbol{\Sigma}_{\boldsymbol{4}}$. We apply Lemma 4.3 with $\Gamma=A_{6}$ (see Table 4.1 ). Conditions (a) and (b) follow from Lemma 4.4(c,b), and conditions (c) and (d) are easily checked. Recall that $U_{i}=P \cap O^{2}\left(G_{i}\right), U=U_{1} U_{2}$, and $Z=T \cap U$. By the lemma, $[T, U]=1$ and $P=U T$; and $U \in \operatorname{Syl}_{2}(\widetilde{\Gamma})$, where $\widetilde{\Gamma}$ is a finite perfect group such that $Z \leq Z(\widetilde{\Gamma})$ and $\widetilde{\Gamma} / Z \cong A_{6}$. Thus $\widetilde{\Gamma}$ is isomorphic to $A_{6} \cong P S L_{2}(9)$ or its 2-fold central extension $S L_{2}(9)$ (cf. [A1,33.15]), and $U \cong D_{8}$ or $Q_{16}$. The image of $U_{i}$ in $P / T \cong U / Z$ is a Sylow 2-subgroup of $O^{2}\left(G_{i} / T\right) \cong A_{4}$, so $P_{i} / T=O_{2}\left(G_{i} / T\right)=U_{i} T / T \cong C_{2}^{2}$, and thus $P_{i}=U_{i} T$. Since each nontrivial subgroup of $Q_{16}$ contains its center,

either $U \cong D_{8}, U_{i} \cong C_{2}^{2}$, and $Z=1$; or $U \cong Q_{16}, U_{i} \cong Q_{8}$, and $|Z|=2$.

Set $T^{\bullet}=T$. We have now proven (i) and (ii.1) in this case, and proven the information on the first two lines in Table 4.2, except for that about $\Delta$.

Case 2: $\boldsymbol{G}_{\boldsymbol{i}} / \boldsymbol{T} \cong \boldsymbol{C}_{\mathbf{2}} \times \boldsymbol{\Sigma}_{\mathbf{4}}$. By Lemma 4.4(a), there are subgroups $\widetilde{G}_{i}<G_{i}$, $\widetilde{P}_{i}<P_{i}$, and $\widetilde{P}<P$ of index two, all containing $T$ and satisfying $(*)$, such that 
$\widetilde{G}_{1}>\widetilde{P}<\widetilde{G}_{2}, \widetilde{P}_{i}=O_{2}\left(\widetilde{G}_{i}\right)$, and $\left(\widetilde{G}_{1} / T>\widetilde{P} / T<\widetilde{G}_{2} / T\right)$ is an amalgam of the type handled in Case 1. Also, $U_{i}=O^{2}\left(G_{i}\right) \cap P=O^{2}\left(\widetilde{G}_{i}\right) \cap \widetilde{P}_{i}$ (i.e., $U_{i}$ and $U$ play the same role for the smaller amalgam as for the original one), and $T$ is not centric in $\widetilde{P}$ by a similar argument to the one used at the end of the proof of Theorem 4.5. So the conclusions in Case 1 hold after replacing $P$ and $P_{i}$ by $\widetilde{P}$ and $\widetilde{P}_{i}$. In particular, (i) holds.

Let $T^{\bullet} / T \leq P_{1} / T \cong C_{2}^{3}$ be the subgroup of order two which is fixed by the conjugation action of $G_{1} / P_{1} \cong \Sigma_{3}$. Then $P_{1} / T=\left(T^{\bullet} / T\right) \cdot\left(T U_{1} / T\right)$ and $P_{2} / T=$ $\left(T^{\bullet} / T\right) \cdot\left(T U_{2} / T\right)$ (but note that $T^{\bullet} / T$ is not fixed by the action of $\operatorname{Aut}_{G_{2}}\left(P_{2}\right)$ on $\left.P_{2} / T\right)$. So $P_{1}=T^{\bullet} U_{1}, P_{2}=T^{\bullet} U_{2}$, and $T^{\bullet} \cap U=T \cap U=Z$. This finishes the proof of (ii.1).

Now, $\left[T^{\bullet}, U\right] \leq T^{\bullet} \cap U=Z$ since $T^{\bullet}$ and $U$ are both normal in $P$. Hence $\left[T^{\bullet}, U\right]=1$ if $|Z|=1$. Now assume $|Z|=2, U \cong Q_{16}$, and $W=\operatorname{Fr}(U) \cong C_{4}$, and fix $g \in T^{\bullet} \backslash T$. Since $Z$ is central in $U,[g,-]$ is a homomorphism from $U$ to $Z$, and so $[g, W]=1$. Also, $g T \in Z\left(G_{1} / T\right)$ by definition of $T^{\bullet}$. Choose $a \in G_{1}$ such that $a T$ has order three in $G_{1} / T$ and $W \neq a W a^{-1}$. Then $a g a^{-1} \in g T, a W a^{-1} \leq P \cap O^{2}\left(G_{1}\right)=U_{1}$, and $\left[g, a W a^{-1}\right]=\left[a g a^{-1}, a W a^{-1}\right]=1$ since $[T, U]=1$ by (i) and $[g, W]=1$. Since $U_{1}=\left\langle W, a W a^{-1}\right\rangle$, we conclude that $\left[T^{\bullet}, U_{1}\right]=\left[g, U_{1}\right]=1$.

By a similar argument, there is $g^{\prime} \in P_{2} \backslash T$ such that $g^{\prime} T \in P_{2} / T \cong C_{2}^{3}$ is the involution fixed by the action of $G_{2} / P_{2} \cong \Sigma_{3}$, and such that $\left[g^{\prime}, U_{2}\right]=1$. Then $g T$ and $g^{\prime} T$ are both in the center of $P / T \cong C_{2} \times D_{8}$, not in $[P / T, P / T]=W T / T$, and $g T \neq g^{\prime} T$ since otherwise $\langle T, g\rangle$ would be normal in $G_{1}$ and in $G_{2}$ (contradicting the maximality of $T)$. So $g^{\prime} \in g w T$ for some $w \in W \backslash Z$. Thus $\left[g w, U_{2}\right]=1$, and since $\left[w, U_{2}\right]=Z\left(w\right.$ has order 4 in $\left.U_{2} \cong Q_{8}\right),\left[T^{\bullet}, U\right]=\left[g, U_{2}\right]=Z$.

This finishes the proof of the information in Table 4.2, except for that about $\Delta$.

Both cases. If $U \cong D_{8}$, then by Lemma B.4, $\Delta \unlhd S_{*}$ is dihedral, $T^{\bullet} \cap \Delta=1$, $\left[S_{*}: T^{\bullet} \Delta\right]=2$, and all noncentral involutions in $\Delta$ are $S_{*}$-conjugate. Fix $y \in U_{1} \backslash Z(U)$. For $g \in S_{*}, c_{g}(y)$ is $\Delta$-conjugate to $y$ if $g \in T^{\bullet} \Delta$ (recall $\left[T^{\bullet}, U_{1}\right]=1$ ), so $c_{g}(y)$ lies in the other $\Delta$-conjugacy class of noncentral involutions if $g \notin T^{\bullet} \Delta$. Thus $C_{S_{*}}\left(U_{1}\right)=$ $C_{T \bullet \Delta}\left(U_{1}\right)=T^{\bullet} U_{1}$.

If $U \cong Q_{16}$, then by Lemma B.4 applied to $U / Z \leq S_{*} / Z, \Delta / Z \unlhd S_{*} / Z$ is dihedral, $T^{\bullet} \cap \Delta=Z,\left[S_{*}: T^{\bullet} \Delta\right]=2$, and all noncentral involutions in $\Delta / Z$ are $S_{*} / Z$-conjugate. Since all involutions in $\Delta / Z$ are $S_{*} / Z$-conjugate to elements of $U_{1} / Z$ where $U_{1} \cong Q_{8}$, there are no involutions in $\Delta \backslash Z$, and $\Delta$ is quaternion. Since $\left[T^{\bullet}, U_{1}\right]=1$, and since the non-normal subgroups of order four in $\Delta$ are $S_{*}$-conjugate, a similar argument to that used in the last paragraph (applied with $y \in U_{1} \backslash \operatorname{Fr}(U)$ ) shows that $C_{S_{*}}\left(U_{1}\right)=$ $C_{T \cdot \Delta}\left(U_{1}\right)=T^{\bullet}$.

This proves (ii.2), and the description of $\Delta$ in Table 4.2 .

(c) Assume $S>S_{*}$, so $N_{S}\left(S_{*}\right)>S_{*}$ by Lemma A.1. Fix $y \in N_{S}\left(S_{*}\right) \backslash S_{*}$ such that $y^{2} \in S_{*}$. Set $\Delta^{*}=y \Delta y^{-1}, Z^{*}=y Z y^{-1}, W^{*}=y W y^{-1}$, and $N=\Delta \cap \Delta^{*}$. The subgroups $\Delta$ and $\Delta^{*}$ are both normal in $S_{*}$, so $N \unlhd S_{*}$ is normal in each of them, and $\left[\Delta, \Delta^{*}\right] \leq N$. If $Z=1$ (if $\Delta$ is dihedral), then $W^{*} \neq W, W^{*} \leq Z\left(S_{*}\right)$ (since $\left.W \leq Z\left(S_{*}\right)\right)$ and hence $W^{*} \not \leq \Delta$, and $N=1$ since each nontrivial normal subgroup of $\Delta^{*}$ contains $W^{*}$. Thus $\left[\Delta^{*}, \Delta\right]=1, \Delta^{*} \leq C_{S_{*}}(\Delta) \leq T^{\bullet} W$, and thus $T^{\bullet} \cong T^{\bullet} W / W$ contains a dihedral subgroup of order 8 . Also, since the commutator subgroup of $\langle\Delta, y\rangle$ contains all elements of the form $g^{-1}\left(y g y^{-1}\right)$ for $g \in \Delta$, it surjects onto $\Delta$ under the projection from $\Delta \cdot \Delta^{*}$, and thus is nonabelian. So $[S, S]$ is nonabelian in this case. 
If $|Z|=2$ (if $\Delta$ is quaternion) and $Z^{*} \neq Z$, then a similar argument shows that $\Delta^{*} \leq T^{\bullet}$ and hence $T^{\bullet}$ contains a quaternion subgroup of order 16 . If $Z^{*}=Z$, we apply the argument in the last paragraph to the conjugation action of $y$ on $S_{*} / Z$, where $y(W / Z) y^{-1} \neq(W / Z)$ by assumption. Thus $\left[\Delta^{*}, \Delta\right] \leq Z$, so $\Delta^{*} \leq C_{S_{*}}\left(U_{1}\right) \cdot W=T^{\bullet} W$ since $\operatorname{Ker}[\operatorname{Aut}(\Delta) \rightarrow \operatorname{Aut}(\Delta / Z)] \cong C_{2}^{2}$ is generated by $\operatorname{Aut}_{W}(\Delta)$ and an automorphism which is the identity on $U_{1}$. So in this case, $T^{\bullet} W / W \cong T^{\bullet} / Z$ contains a dihedral subgroup of order 8 . In both cases, $[S, S]$ is nonabelian by the argument used in the last paragraph.

(b) If $T$ is centric in $P$, then it is centric in $S$ by Lemma $4.2(\mathrm{~d})$, while the converse is immediate. Assume both of these hold.

If $T$ is not fully normalized, then there is some $\varphi \in \operatorname{Hom}_{\mathcal{F}}\left(N_{S}(T), S\right)$ such that $\varphi(T)$ is fully normalized (cf. [AKO, Lemma I.2.6(c)]). Upon replacing $T$ by $\varphi(T), P_{i}$ by $\varphi\left(P_{i}\right), P$ by $\varphi(P)$, etc., we can assume $T$ is fully normalized (and $(*)$ still holds and $T$ is still centric in $P$ ).

Set $\widehat{S}=\operatorname{Out}_{S}(T), \widehat{G}_{i}=\operatorname{Out}_{G_{i}}(T)$, and similarly for subgroups of $S$, and set $\widehat{G}=$ $\left\langle\widehat{G}_{1}, \widehat{G}_{2}, \widehat{S}\right\rangle \leq \operatorname{Out}_{\mathcal{F}}(T)$. Then $\widehat{S} \in \operatorname{Syl}_{2}(\widehat{G})$ by the Sylow axiom. Set $V=T / \operatorname{Fr}(T)$, written additively, and regarded as an $\mathbb{F}_{2}[\operatorname{Out}(T)]$-module. By Lemma $4.2(\mathrm{e}), \mathrm{O}_{2}(\widehat{G})=$ 1 and $\widehat{G}$ acts faithfully on $V$. Hence $O_{2}\left(\operatorname{Out}_{\mathcal{F}}(T)\right)=1$, and by Lemma A.2, $\operatorname{Out}_{\mathcal{F}}(T)$ acts faithfully on $V$.

It remains to show that $[S, S]$ is nonabelian. Set $V_{0}=[\widehat{S}, V]$. Since every element of $\widehat{S}$ lifts to an element of $S$, and every element of $V_{0}$ lifts to an element of $[S, S]$, it will suffice to show that $[\widehat{S}, \widehat{S}]$ acts nontrivially on $V_{0}$. Assume otherwise: assume $[\widehat{S}, \widehat{S}]$ acts trivially on $V_{0}$. If $g \in[\widehat{S}, \widehat{S}]$, then for each $v \in V, g$ acts trivially on $[g, v]=g v-v \in V_{0}$, so $(g-1)^{2} v=\left(g^{2}-1\right) v=0$, and $g^{2} v=v$. Thus $g^{2}=1$ since $\widehat{S}$ acts faithfully on $V$. It follows that $[\widehat{S}, \widehat{S}]$ is elementary abelian.

If $\widehat{G}_{1} \cong \widehat{G}_{2}$ are strictly 2-constrained, then by the argument used in the second paragraph in the proof of Theorem 4.5, $\left(\widehat{G}_{1}>\widehat{P}<\widehat{G}_{2}\right)$ must be one of the amalgams listed in Table 4.1. Hence $\widehat{P} \cong D_{8}$ or $D_{8} \times C_{2}$ (the only cases in the table with $\left.\bar{G}_{1} \cong \bar{G}_{2}\right)$, and $\widehat{P}_{i}=O_{2}\left(\widehat{G}_{i}\right) \cong C_{2}^{2}$ or $C_{2}^{3}$, respectively. Note that $\widehat{P}_{12}=Z(\widehat{P})$ in either case. Choose $x_{1} \in \widehat{P}_{1} \backslash \widehat{P}_{12}$. Then $x_{1}$ is $\widehat{S}$-conjugate to some $x_{2} \in \widehat{P}_{2} \backslash \widehat{P}_{12}$, and $x_{1} x_{2}^{-1} \in[\widehat{S}, \widehat{S}]$ has order 4 . So this case is impossible.

Thus $\widehat{G}_{1} \cong \widehat{G}_{2}$ are not strictly 2-constrained. By Lemma $4.2(\mathrm{c}), O_{p}\left(\widehat{G}_{i}\right) \neq 1$ for $i=1,2$. Thus $\left[\widehat{P}_{i}, O_{p}\left(\widehat{G}_{i}\right)\right]=1$ since both are normal in $\widehat{G}_{i}$ (and have relatively prime order), so $\widehat{P}_{12} \unlhd \widehat{G}_{i}$ for $i=1,2$. Hence $P_{12} \unlhd G_{i}$, and $P_{12}=T$ by definition of $T$. In other words, $\widehat{P}_{12}=1, \widehat{P}_{i} \cong C_{2}$, and so $\widehat{P} \cong C_{2}^{2}$.

Let $a_{i} \in \widehat{P}_{i} \cong C_{2}$ be the generator, and let $g \in O_{p}\left(\widehat{G}_{1}\right)$ be an element of order $p$. Thus $\widehat{G}_{1}=\left\langle a_{1}, a_{2}, g\right\rangle \cong D_{4 p},\left\langle g, a_{2}\right\rangle \cong D_{2 p}$, and $\left[g, a_{1}\right]=1$. Choose $t \in \widehat{S}$ such that $t a_{i} t^{-1}=a_{3-i}$. By assumption, and since $a_{1} a_{2} \in[\widehat{S}, \widehat{S}],\left[a_{1} a_{2},[\widehat{S}, V]\right]=0$.

Set $W=[g, V] ; W \neq 0$ since $\widehat{G}_{1}$ acts faithfully on $V$. We claim that

$$
C_{W}\left(a_{2}\right)=\left[a_{2}, W\right] \quad \text { and } \quad\left[a_{1}, W\right]=0 .
$$

If $w \in C_{W}\left(a_{2}\right)$, then $a_{2}\left(g^{i} w\right)=g^{-i} a_{2} w=g^{-i} w$ for each $i$, and $\sum_{i=0}^{p-1} g^{i} w=0$ since $w \in$ $W=[g, V]$. Thus $w=\left(1+a_{2}\right)\left(\sum_{i=1}^{(p-1) / 2} g^{i} w\right) \in\left[a_{2}, W\right]$, proving the first statement, and in fact proving that $C_{W_{0}}\left(a_{2}\right)=\left[a_{2}, W_{0}\right]$ for each $\widehat{G}_{1}$-invariant subgroup $W_{0} \leq W$. 
So if we set $W_{0}=\left[a_{1}, W\right]$, then $C_{W_{0}}\left(a_{2}\right)=\left[a_{2}, W_{0}\right]=\left[a_{1} a_{2}, W_{0}\right] \leq\left[a_{1} a_{2},[\widehat{S}, V]\right]$, where the last group is zero by assumption. Hence $W_{0}=0$, finishing the proof of $(1)$.

Since $W=[g, V]$ is a $\widehat{G}_{1}$-invariant direct summand of $V, W \cap\left[a_{1}, V\right]=\left[a_{1}, W\right]=0$ by (1). So if $v \in W \cap t(W)$, then $a_{1}(v)=v$ since $v \in W, v \in C_{t(W)}\left(a_{1}\right)=\left[a_{1}, t(W)\right]$ by (1), and so $v \in W \cap\left[a_{1}, V\right]=0$. Thus $W \cap t(W)=0$. So if we choose any $w \in W$ such that $a_{2}(w) \neq w$, then $t(w)-w \in[\widehat{S}, V]$, and hence

$$
a_{1} a_{2}(t(w)-w)=t(w)-w \Longrightarrow w-a_{1} a_{2}(w)=t(w)-t a_{1} a_{2}(w) \in W \cap t(W)=0 \text {. }
$$

Thus $a_{1}(w) \neq a_{1} a_{2}(w)=w$, which contradicts (1).

\section{SOME APPliCATiONS}

We finish the paper with some applications of Theorems 4.5 and 4.6. Following the terminology of [G, $\S 16.7$, we say that a 2 -group $S$ is wreathed if $S \cong C_{2^{m}} \succ C_{2}$ for some $m \geq 2$. These groups arise as Sylow 2-subgroups of $G L_{2}(q)$ and $(P) S L_{3}(q)$ when $q \equiv 1$ $(\bmod 4)$.

Proposition 5.1. Fix a finite 2-group $S$ containing a normal subgroup $\Delta \unlhd S$ which is dihedral or quaternion of order $\geq 8$. Assume

(a) for some dihedral or quaternion subgroup $\Delta_{0} \leq \Delta$ of order $8, C_{S}\left(\Delta_{0}\right)$ is abelian; and

(b) two of the three subgroups of index two in $\Delta$ are $S$-conjugate.

Then either $S$ is dihedral, semidihedral, or wreathed, or there is no reduced fusion system over $S$.

Proof. We first fix some notation for elements and subgroups of $S$. Let $A \unlhd \Delta$ be the unique cyclic subgroup of index two in $\Delta$ which is normal in $S$ ( $A$ is characteristic in $\Delta$ unless $\left.\Delta \cong Q_{8}\right)$. Set $Z=Z(\Delta)$, and let $W \leq A$ be the subgroup of order 4 . Fix a generator $a \in A$, and fix $b \in \Delta_{0} \backslash A$. Thus $\Delta=\langle a, b\rangle$ and $\Delta_{0}=\langle W, b\rangle$. Set $A_{0}=\left\langle a^{2}\right\rangle$, and set $T=C_{S}\left(\Delta_{0}\right)$. To summarize these definitions for later reference,

$$
\underset{=Z(\Delta)}{Z} \leq \underset{=\left\langle a^{2}\right\rangle}{A_{0}}<\underset{=\langle a\rangle}{A}<\underset{=\langle a, b\rangle}{\Delta} \unlhd S, \quad \underset{\cong C_{4}}{W} \leq A, \quad \Delta_{0}=\langle W, b\rangle, \quad T=C_{S}\left(\Delta_{0}\right) .
$$

By (a) and (b), we are in the situation of Lemma B.3. In particular,

$$
[S: T \Delta]=2 \quad \text { and } \quad \forall g \in S, g b g^{-1}=a^{j} b \text { where } \begin{cases}j \text { is even } & \text { if } g \in T \Delta \\ j \text { is odd } & \text { if } g \notin T \Delta\end{cases}
$$

by Lemma B.3(b). Note also that

$$
\forall g \in S, g a g^{-1}=a^{i} \quad \text { where } \begin{cases}i \in 4 \mathbb{Z}+1 & \text { if } g \in C_{S}(W) \\ i \in 4 \mathbb{Z}-1 & \text { if } g \notin C_{S}(W) .\end{cases}
$$

We claim that the following hold.

$$
\begin{gathered}
T A_{0} \unlhd S, \quad S / T A_{0} \cong D_{8}, \quad \text { and } Z\left(S / T A_{0}\right)=T A / T A_{0} . \\
{[S, S] \text { is abelian, and }[S, S]=\langle A,[y, T]\rangle \text { for each } y \in S \backslash T \Delta .} \\
{[S, S]=A \Longrightarrow \forall g \in S \backslash C_{S}(W), C_{T A}(g) \cdot A=T A \text { and }\left|C_{T A}(g)\right|=|T| .}
\end{gathered}
$$


Point (3) and the first statement in (4) are shown in Lemma B.3(c,d). The last statement in (4) holds since $[S, S] \geq A$ by Lemma B.3(a), $S=\langle\Delta, T, y\rangle$, and hence $[S, S]=\langle[\Delta, S],[y, T]\rangle=\langle A,[y, T]\rangle$.

To see (5), fix $g \in S \backslash C_{S}(W)$ and $t \in T$. By (2), $g a g^{-1}=a^{4 k-1}$ for some $k \in \mathbb{Z}$. Also, $t^{-1} g t=a^{j} g$ for some $j$ since $[S, S]=A$, and $j$ is even $\left(a^{j} \in A_{0}\right)$ since $T A_{0} \unlhd S$ by (3). Choose $i$ such that $i(1-2 k) \equiv(j / 2)(\bmod |A|)$. Then $a^{i} g a^{-i}=a^{j} g=t^{-1} g t$, so $t a^{i} \in C_{T A}(g)$. This proves the first statement in (5), and the second then follows since $C_{T A}(g) \cap A=C_{A}(g)=Z=T \cap A$.

Set $\bar{S}=S / T A_{0}\left(T A_{0} \unlhd S\right.$ by (3)). We write $\bar{g}=g T A_{0} \in \bar{S}$ for $g \in S$, and $\bar{Q}=Q T A_{0} / T A_{0}$ for $Q \leq S$ : the images of $g$ and $Q$ in $\bar{S}$.

Fix $x \in S \backslash T \Delta$. Upon replacing $x$ by $b x$ if necessary, we can assume $x \in C_{S}(W) \backslash T \Delta$. Also, $x b x^{-1}=a^{j} b$ for some odd $j$ by (1), and upon replacing $x$ by an appropriate element of $x A$, we can assume $x b x^{-1}=a b$. By (2), $x a x^{-1}=a^{i}$ for some $i \in 1+4 \mathbb{Z}$. Then $x^{2} b x^{-2}=a^{i+1} b$ where $i+1 \in 2+4 \mathbb{Z} ; x^{2} \in T A$ since $S / T A \cong \bar{S} / Z(\bar{S}) \cong C_{2}^{2}$ by $(3) ;$ and hence $x^{2} \in T A_{0} a$. To summarize,

$$
S=\langle T A, b, x\rangle, \quad C_{S}(W)=\langle T A, x\rangle, \quad x b x^{-1}=a b, \quad \bar{x}^{2}=\bar{a} \in \bar{S} .
$$

Assume $\mathcal{F}$ is a reduced fusion system over $S$, and let $\mathcal{E}$ be the set of $\mathcal{F}$-essential subgroups of $S$. Define

$$
b_{0}=x, \quad b_{1}=b, \quad b_{2}=b x, \quad H_{i}=\left\langle T A, b_{i}\right\rangle, \quad \mathcal{E}_{i}=\left\{P \in \mathcal{E} \mid P \leq H_{i}\right\}
$$

for $i=0,1,2$. Thus $H_{0}=C_{S}(W)$ and $H_{1}=T \Delta$. We will prove the following statements.

$$
\begin{gathered}
\mathcal{E}=\mathcal{E}_{0} \cup \mathcal{E}_{1} \cup \mathcal{E}_{2}, \quad \text { and } P \in \mathcal{E}_{i} \Longrightarrow \text { the normal closure of } P \text { is } H_{i} \\
\qquad \mathcal{E}_{0} \neq \varnothing \Longrightarrow S \text { is wreathed. } \\
\mathcal{E}_{2} \neq \varnothing \Longrightarrow[S, S]=A, \exists b^{\bullet} \in T A b_{2} \text { s.t. }\left\langle A, b^{\bullet}\right\rangle \text { dihedral or quaternion. } \\
\mathcal{E}_{0}=\varnothing \Longrightarrow \mathcal{E}_{1} \neq \varnothing, \mathcal{E}_{2} \neq \varnothing, \text { and } S \text { is dihedral or semidihedral. }
\end{gathered}
$$

The proposition then follows from (8) and (10).

Points (7), (8), and (9) will be shown in Steps 1 and 2: essential subgroups of index two in $S$ will be handled in Step 1, and the others in Step 2. It is in Step 2 that Theorem 4.6 plays a crucial role. Point (10) will be shown in Step 3.

Step 1: Fix $P \in \mathcal{E}$ such that $[S: P]=2$. Thus $P \geq[S, S] \geq A$.

We consider seven cases. In the first four, we show $P$ cannot be $\mathcal{F}$-essential using Lemma 1.4 applied with $\Theta$ one of the following characteristic subgroups $\Theta_{i} \leq P$ :

$$
\Theta_{1}=Z(P), \quad \Theta_{2}=\left\langle g \in P \mid\left[P: C_{P}(g)\right] \leq 2\right\rangle, \quad \text { or } \quad \Theta_{3}=\langle\beta(A) \mid \beta \in \operatorname{Aut}(P)\rangle .
$$

By Lemma 1.4, $P \notin \mathcal{E}$ if for some $i=1,2,3$ and some $g \in S \backslash P,[g, P] \leq \Theta_{i} \operatorname{Fr}(P)$ and $\left[g, \Theta_{i}\right] \leq \operatorname{Fr}(P)$.

Since $P \geq A$, either $P \nsupseteq T$, or $P$ is one of the $H_{i}$. In the first two cases below, $P \nsupseteq T$, and we set $T_{0}=P \cap T$, choose $t^{\bullet} \in T \backslash T_{0} \subseteq S \backslash P$, and let $t_{1}, t_{2} \in\left\{1, t^{\bullet}\right\}$ be such that $P=\left\langle T_{0} A, b t_{1}, x t_{2}\right\rangle$. Since $\left[\overline{x t_{2}}, \overline{b t_{1}}\right]=[\bar{x}, \bar{b}]=\bar{a},\left[x t_{2}, b t_{1}\right] \in[S, S] \cap T A_{0} a$.

- $\boldsymbol{P} ¥ \boldsymbol{T},\left[\boldsymbol{t}^{\bullet}, \boldsymbol{P}\right] \leq \boldsymbol{A} Z(\boldsymbol{S})$, and $\Theta=\Theta_{1}=\boldsymbol{Z}(\boldsymbol{P})$. Then $\bar{P}=\bar{S}$, so $\overline{Z(P)} \leq Z(\bar{S})=\overline{T A}$ by $(3)$, and $Z(P) \leq T A$. Thus $\left[t^{\bullet}, Z(P)\right] \leq\left[t^{\bullet}, T A\right] \leq$ $\left\langle a^{4}\right\rangle \leq \operatorname{Fr}(P)$. Also, $\left[t^{\bullet}, P\right] \leq\left[t^{\bullet}, S\right] \leq T A_{0}$ since $t^{\bullet} \in T A_{0} \unlhd S$ by (3). So 
by assumption (and since $Z(S) \leq C_{S}\left(\Delta_{0}\right)=T$ ), $\left[t^{\bullet}, P\right] \leq T A_{0} \cap A Z(S)=$ $Z(S) A_{0} \leq \Theta_{1} \operatorname{Fr}(P)$. Thus $P \notin \mathcal{E}$.

- $\boldsymbol{P} \ngtr \boldsymbol{T},\left[\boldsymbol{t}^{\bullet}, \boldsymbol{P}\right] \not \mathbf{A} \boldsymbol{Z}(\boldsymbol{S})$, and $\boldsymbol{\Theta}=\boldsymbol{\Theta}_{\mathbf{2}}$. Then $\left[t^{\bullet}, P\right] \leq T A_{0} \cap[S, S]$ since $t^{\bullet} \in T A_{0} \unlhd S$. Since $\left[t^{\bullet}, T \Delta\right]=\left[t^{\bullet}, A\right] \leq A_{0}$ (recall $\langle T, b\rangle$ is abelian) and $\left[t^{\bullet}, P\right] \not \leq A Z(S),\left[t^{\bullet}, x\right]=\left[t^{\bullet}, x t_{2}\right]=s a^{2 i}$ for some $s \in T_{0} \backslash A Z(S)$. Then $[s, A]=1$ since $s a^{2 i} \in[S, S], A \leq[S, S]$, and $[S, S]$ is abelian by (4). Hence $[s, T \Delta]=1$, and $[s, g] \neq 1$ for $g \in S \backslash T \Delta$ since $s \notin Z(S)$.

Now, $s \in \Theta$ since $C_{P}(s)=T \Delta \cap P$. Also, $\left[t^{\bullet}, T \Delta\right] \leq A_{0} \unlhd S$, so $\left[t^{\bullet}, P\right] \leq$ $\left\langle A_{0},\left[t^{\bullet}, x\right]\right\rangle=\left\langle A_{0}, s\right\rangle \leq \Theta \operatorname{Fr}(P)$. For all $g \in P \backslash T \Delta, C_{P}(g) \leq\left\langle T_{0} A, g\right\rangle$ since $C_{\bar{S}}(\bar{g})=\langle\bar{a}, \bar{g}\rangle$, and this inclusion is strict since $[s, g] \neq 1$. Thus $g \notin \Theta$. So $\Theta \leq T \Delta$, and hence $\left[t^{\bullet}, \Theta\right] \leq[T, T \Delta] \leq\left\langle a^{4}\right\rangle \leq \operatorname{Fr}(P)$. Thus $P \notin \mathcal{E}$.

- $\boldsymbol{P} \in\left\{\boldsymbol{H}_{\mathbf{0}}, \boldsymbol{H}_{\mathbf{2}}\right\}$ and $\boldsymbol{\Theta}=\boldsymbol{\Theta}_{\mathbf{3}} \leq \boldsymbol{T} \boldsymbol{A}$. Then $b \in S \backslash P,[b, P] \leq A \leq \Theta$ since $b A \in Z(S / A)$, and $[b, \Theta] \leq[b, \bar{T} A] \leq A_{0} \leq \operatorname{Fr}(P)$. Thus $P \notin \mathcal{E}$.

- $\boldsymbol{P}=\boldsymbol{H}_{1},[\boldsymbol{S}, \boldsymbol{S}]=\boldsymbol{A}$, and $\boldsymbol{\Theta}=\boldsymbol{\Theta}_{\mathbf{3}} \leq \boldsymbol{T} \boldsymbol{A}$. Then $x \in S \backslash P$ and $[x, P] \leq[S, S]=$ $A \leq \Theta$. Also, since $\overline{T A}=Z(\bar{S})$ and hence $[S, T A] \leq T A_{0},[x, \Theta] \leq[S, T A] \leq$ $A \cap T A_{0}=A_{0} \leq \operatorname{Fr}(P)$. Thus $P \notin \mathcal{E}$.

- $\boldsymbol{P}=\boldsymbol{H}_{1},[\boldsymbol{S}, \boldsymbol{S}]>\boldsymbol{A}$, and $\Theta_{3} \leq \boldsymbol{T} \boldsymbol{A}$. We will see later that we don't need to consider this case separately.

- $\boldsymbol{P}=\boldsymbol{H}_{\mathbf{0}}$, and $\boldsymbol{\Theta}_{\mathbf{3}} \not \mathbf{T} \boldsymbol{A}$. Since $\Theta_{3} \not \leq T A$, there is $\beta \in \operatorname{Aut}(P)$ such that $\beta(A) \not T A$. Set $u=\beta(a) \in P \backslash T A=T A x$ and $U=\langle u\rangle=\beta(A) \unlhd P$. Then $\bar{u} \in\{\bar{x}, \overline{a x}\}$, so $\bar{u}^{2}=\bar{a}$ by (6), and $u^{2}=t a^{i}$ where $t \in T$ and $i$ is odd. Set $C=A \cap U$ and $2^{m}=[A: C]=[U: C]$, so $[A, U] \leq C, C=\left\langle a^{2^{m}}\right\rangle=\left\langle u^{2^{m}}\right\rangle$, and $A U / C \cong\left(C_{2^{m}}\right)^{2}$. Then $t^{2^{m-1}}=\left(u^{2} a^{-i}\right)^{2^{m-1}} \equiv u^{2^{m}} a^{-i \cdot 2^{m-1}} \equiv a^{2^{m-1}}(\bmod C)$ since $i$ is odd, so $t^{2^{m-1}}=a^{k \cdot 2^{m-1}}$ for some odd $k, a^{k \cdot 2^{m-1}} \in A \cap T=Z$ has order $\leq 2$, and hence $|a|=2^{m}$. Thus $C=1$, and $A U \cong A \times A \cong\left(C_{2^{m}}\right)^{2}$.

Now, $\left[T, u^{2}\right] \leq[T, T A] \leq A$, and $\left[T, u^{2}\right] \leq U$ since $U \unlhd P$. Hence $\left[T, u^{2}\right]=1$, and $[T, A]=\left[T, a^{i}\right]=1$ since $u^{2}=t a^{i}$ for $i$ odd. Thus $[A, P]=[A, T A U]=1$, $[U, P]=\beta([A, P])=1$, and so $P=T A U$ is abelian. Also, $T \leq Z(S)$ since $S=\langle P, b\rangle$ and $[T, b]=1$. Hence $[S, S]=A$ by $(4)$.

Thus $[S, S]=A$ is cyclic and $H_{0}=P$ is abelian of index two in $S$, so we are in the situation of Proposition 2.5. Since $S \geq A U \cong A \times A, S$ is not dihedral or semidihedral, and hence is wreathed.

- $\boldsymbol{P} \in\left\{\boldsymbol{H}_{1}, \boldsymbol{H}_{\mathbf{2}}\right\}$ and $\boldsymbol{\Theta}_{3} \not \mathbf{T} \boldsymbol{A}$. Let $i=1,2$ be such that $P=H_{i}=\left\langle T A, b_{i}\right\rangle$. Since $\Theta_{3} \not \leq T A$, there is $\beta \in \operatorname{Aut}(P)$ such that $\beta(a) \notin T A$. Set $b^{\bullet}=\beta(a)$; thus $b^{\bullet} \in T A b_{i} \subseteq S \backslash C_{S}(W)$, so $b^{\bullet} a b^{\bullet-1}=a^{j}$ for some $j \in-1+4 \mathbb{Z}$ by (2). Then

$$
\beta(A) \geq \beta([a, P])=\left[b^{\bullet}, P\right] \geq\left[b^{\bullet}, A\right]=\left\langle a^{2}\right\rangle=A_{0}
$$

since $A \geq[A, P],\left[\beta(A): A_{0}\right]=2$ since $|\beta(A)|=|A|$, and so $b^{\bullet 2}=a^{2 \ell}$ for some odd $\ell$. Thus $a^{2}=b^{\bullet} a^{2} b^{\bullet-1}=a^{2 j}$, so $j \equiv 1\left(\bmod \frac{1}{2}|a|\right)$. Since $j \equiv 3(\bmod 4)$, this proves that $|a|=\left|b^{\bullet}\right|=4,\left[b^{\bullet}, a\right]=a^{2}$, and thus $\left\langle A, b^{\bullet}\right\rangle=\left\langle a, b^{\bullet}\right\rangle \cong Q_{8}$. Also, $A=W$, so $[A, T]=1$, and $T A$ is abelian.

If $P=H_{2}=\langle T A, b x\rangle$, then $\left[b^{\bullet}, T\right] \leq\left(\left\langle b^{\bullet}\right\rangle \cap T A\right)=Z$ since $\left\langle b^{\bullet}\right\rangle=\beta(A) \unlhd P$. Hence $[S, S]=\left\langle A,\left[b^{\bullet}, T\right]\right\rangle=A$ by $(4)$.

To summarize, if $P \in \mathcal{E}$ has index 2 in $S$, then either $P=H_{0}$ and $S$ is wreathed, or $P=H_{2}$ and $[S, S]=A$, or $P=H_{1}$. Also, if $P \in\left\{H_{1}, H_{2}\right\}$ and $[S, S]=A$, then $P=T \Delta^{\bullet}$ for some $Q_{8} \cong \Delta^{\bullet} \unlhd P$, and $P=C_{T A}\left(\Delta^{\bullet}\right) \Delta^{\bullet}$ by (5). So this proves (7), (8), and (9) for essential subgroups of index two in $S$. 
Step 2: Now assume $P \in \mathcal{E}$ where $[S: P]>2$. If $\left|N_{S}(P) / P\right|>2$, then by Lemma $1.5, \operatorname{rk}([g, P / \operatorname{Fr}(P)]) \geq 2$ for all $g \in N_{S}(P) \backslash P$. For each $i \in \mathbb{Z},\left[a^{i}, S\right]=\left\langle a^{2 i}\right\rangle$, so $a^{i} \in N_{S}(P)$ if $a^{2 i} \in P$, which implies $a^{i} \in P$ since $\left[a^{i}, S\right]$ is cyclic. Thus $A \leq P$ by induction on $|A \cap P|$, so $a^{2} \in \operatorname{Fr}(P)$. For $t \in T,[t, S] \leq\left\langle a^{4},[t, x]\right\rangle$, and thus $P \geq T A$. Hence $P=T A$ since $[S: P] \geq 4$, so $[b, P]=\left\langle a^{2}\right\rangle \leq \operatorname{Fr}(P)$ (and $b \in N_{S}(P)$ ), which contradicts Lemma 1.5. We conclude that $\left|N_{S}(P) / P\right|=2$.

In particular, $P$ is not normal in $S$. By Theorem 4.6(b,c), and since $[S, S]$ is abelian by (4), $P$ is of the type described in Theorem 4.6(a) with $S_{*}=S$. Thus $P=C_{S}\left(U^{\bullet}\right) U^{\bullet}$, where $U^{\bullet} \cong C_{2}^{2}$ or $Q_{8}$, and the normal closure $\Delta^{\bullet}$ of $U^{\bullet}$ in $S$ is dihedral of order $\geq 8$ or quaternion of order $\geq 16$. Let $A^{\bullet} \unlhd \Delta^{\bullet}$ be the unique cyclic subgroup of index two, and fix $b^{\bullet} \in U^{\bullet} \backslash A^{\bullet}$. Thus $\Delta^{\bullet}=\left\langle A^{\bullet}, b^{\bullet}\right\rangle$.

Since $A^{\bullet} \leq \Delta^{\bullet}$ are both normal in $S$, the coset $A^{\bullet} b^{\bullet}=\Delta^{\bullet} \backslash A^{\bullet}$ is a union of $S$ conjugacy classes, and its elements are all conjugate since otherwise $\left[b^{\bullet}, S\right]=\left[b^{\bullet}, A^{\bullet}\right]$ has index two in $A^{\bullet}$ and the normal closure of $U^{\bullet}$ is strictly contained in $\Delta^{\bullet}$. Hence

$$
A^{\bullet}=\left[b^{\bullet}, S\right] \leq[S, S] \leq T A
$$

(recall that $S / T A \cong C_{2}^{2}$ is abelian by (3)). We claim that $\Delta^{\bullet} \not \leq T A$, and hence $b^{\bullet} \notin T A$. Assume otherwise: then $Z\left(\Delta^{\bullet}\right) \leq\left[\Delta^{\bullet}, \Delta^{\bullet}\right] \leq[T A, T A] \leq A$, so $Z\left(\Delta^{\bullet}\right)=Z$, and the dihedral group $\Delta^{\bullet} / Z\left(\Delta^{\bullet}\right)$ is generated by elements of order two in $T A / Z$. Since each element of order two in $T A / Z \cong(A / Z) \rtimes(T / Z)$ lies in $T W / Z, \Delta^{\bullet}$ is contained in the abelian group $T W$, which is impossible.

Thus $b^{\bullet} \in H_{i} \backslash T A=T A b_{i}$ for some unique $i=0,1,2$. Since $b^{\bullet} \notin T A, \bar{b}^{\bullet}$ is not central in $\bar{S} \cong D_{8}$ (see $(3)$ ), so $C_{\bar{S}}\left(\overline{b^{\bullet}}\right)=\left\langle\overline{b^{\bullet}}, \bar{a}\right\rangle=\bar{H}_{i}$. Hence $C_{S}\left(b^{\bullet}\right) \leq H_{i}=\left\langle T A, b^{\bullet}\right\rangle$, so $P=C_{S}\left(U^{\bullet}\right) \cdot U^{\bullet} \leq C_{S}\left(b^{\bullet}\right) A^{\bullet} \leq H_{i}$, and $P \in \mathcal{E}_{i}$. Since the normal closure of $P$ has index two in $S=S_{*}$ by Theorem 4.6(a) again, it must be equal to $H_{i}$. This proves (7).

Assume $i=0\left(\right.$ so $\left.b^{\bullet} \in T A x\right)$, or $i=2\left(\right.$ so $\left.b^{\bullet} \in T A b x\right)$. Since $[b, T A]=A_{0}=\left\langle a^{2}\right\rangle$ and $[x, b]=a$, we get $\left\langle\left[b^{\bullet}, b\right]\right\rangle=A$ in all cases. Thus $A^{\bullet}=\left[b^{\bullet}, S\right] \geq A, \overline{A^{\bullet}}=[\bar{S}, \bar{S}]=\bar{A}$, and hence $A^{\bullet}=A$. (If $A^{\bullet}>A$, then since both are cyclic, they could not have the same nontrivial image in any quotient group.) So $\Delta^{\bullet}=\left\langle A, b^{\bullet}\right\rangle$. Also, since $S=\left\langle T \Delta, b^{\bullet}\right\rangle$ in these cases, (4) implies that

$$
[S, S]=\left\langle A,\left[b^{\bullet}, T\right]\right\rangle=A .
$$

Together with Step 1, this proves (9).

The subgroup $W \leq A^{\bullet}=A$ of order 4 is central in $H_{0}$ and not central in $\Delta^{\bullet}$. Thus $P \notin \mathcal{E}_{0}$. Together with Step 1, this finishes the proof of (8): $\mathcal{E}_{0}=\varnothing$ or $\mathcal{E}_{0}=\left\{H_{0}\right\}$, and in the latter case, $S$ is wreathed.

Step 3: We have now proven (7), (8), and (9), and it remains to prove (10). So assume $\mathcal{E}_{0}=\varnothing$. Then $\mathcal{E}_{1} \neq \varnothing$ and $\mathcal{E}_{2} \neq \varnothing$ by (7) and Lemma 2.2 , and $[S, S]=A$ by (9). It remains to prove that $S$ is dihedral or semidihedral.

Fix $P_{2}=C_{S}\left(U^{\bullet}\right) U^{\bullet} \in \mathcal{E}_{2}$ with normal closure $H_{2}$, as described in Step 2. Choose $b^{\bullet} \in U^{\bullet} \backslash A$, so that $\left\langle A, b^{\bullet}\right\rangle$ is the normal closure of $U^{\bullet}$ and is dihedral or quaternion. Thus $b^{\bullet} \in T A b x$, and $\widehat{\Delta} \stackrel{\text { def }}{=}\left\langle A, b, b^{\bullet}\right\rangle \unlhd S$ is dihedral, semidihedral, or quaternion by Lemma B.3(e). Also, $T \widehat{\Delta}=\left\langle T A, b, b^{\bullet}\right\rangle=S$. Let $\widehat{A}<\widehat{\Delta}$ be the cyclic subgroup of index two (so $\widehat{\Delta}=\langle\widehat{A}, b\rangle,[\widehat{A}: A]=2$, and $\widehat{A} \unlhd S$ ).

If $S$ contains an abelian subgroup of index two, then since $[S, S]=A$ is cyclic, $S$ is dihedral, semidihedral, or wreathed by Proposition 2.5. Since $[S, S]=A$ is strictly contained in the larger cyclic subgroup $\widehat{A}, S$ cannot be wreathed. 
Assume $S$ does not contain an abelian subgroup of index two. Thus $T \widehat{A}$ is nonabelian, so $[T, \widehat{A}] \neq 1(T$ and $\widehat{A}$ are both abelian), and the homomorphism $c: T \longrightarrow \operatorname{Aut}(\widehat{A})$ induced by conjugation is nontrivial. Also, $\operatorname{Im}(c)$ is cyclic (each $t \in T$ acts via $\left(a \mapsto a^{i}\right)$ for $i \in 1+4 \mathbb{Z}$ by $(2))$, and $C_{T}(\widehat{A})=\operatorname{Ker}(c)=Z(S)$ since $S=\langle T \widehat{A}, b\rangle,[T, T]=[T, b]=$ 1 , and $T \stackrel{\text { def }}{=} C_{S}\left(\Delta_{0}\right) \geq Z(S)$. Also, $C_{T}(A)=\{t \in T|| c(t) \mid \leq 2\}$, since for $i \in 4 \mathbb{Z}+1$, $i \equiv 1(\bmod |A|)$ if and only if $i^{2} \equiv 1(\bmod |\widehat{A}|)$. So $\left[C_{T}(A): C_{T}(\widehat{A})\right]=2$. Thus

$$
C_{S}(A) / Z(S)=C_{T}(A) \widehat{A} / C_{T}(\widehat{A}) \cong\left(C_{T}(A) / C_{T}(\widehat{A})\right) \times(\widehat{A} / Z) \cong C_{2} \times(\widehat{A} / Z)
$$

since $\left[C_{T}(A), \widehat{A}\right]=Z$, so $\operatorname{Aut}\left(C_{S}(A) / Z(S)\right)$ is a 2-group by Corollary A.3(a). Hence each element of odd order in $\operatorname{Aut}_{\mathcal{F}}(S)$ induces the identity on $C_{S}(A) / Z(S)$, and so the action of the odd order group $\operatorname{Out}_{\mathcal{F}}(S)$ on $S^{\mathrm{ab}}=S / A$ induces the identity on $C_{S}(A) / Z(S) A$.

We want to apply Proposition 2.3(b), with $U=Z=[S, S] \cap Z(S)$. For each $P<S$ of index two, $1 \neq[P, P] \leq[S, S]=A$, so $[P, P] \geq Z$ since $A$ is cyclic. Choose $g_{0} \in C_{T}(A) \backslash C_{T}(\widehat{A})$, and let $g_{1}$ be such that $g_{1} A=\prod_{\alpha \in \operatorname{Out}_{\mathcal{F}}(S)} \alpha\left(g_{0} A\right)$. Since $\operatorname{Out}_{\mathcal{F}}(S)$ acts trivially on $C_{S}(A) / Z(S) A, g_{1} \equiv g_{0}^{k}(\bmod Z(S) A)$, where $k=\left|\operatorname{Out}_{\mathcal{F}}(S)\right|$ is odd, and we can assume $g_{1} \in T$ and $g_{1} \equiv g_{0}^{k}(\bmod Z(S))$. Thus $\left|c\left(g_{1}\right)\right|=\left|c\left(g_{0}^{k}\right)\right|=2$ since $k$ is odd and $\left[g_{0}, \widehat{A}\right]=Z$, so $g_{1} \notin Z$. Fix $g \in\left\langle g_{1}\right\rangle$ such that $g \notin Z$ but $g^{2} \in Z$. Then $|c(g)| \leq 2$, so $[g, S]=[g, \widehat{A}] \leq Z$. By construction, every element of $\operatorname{Aut}_{\mathcal{F}}(S)$ sends $g A$ to itself.

Thus $\mathcal{F}$ is not reduced by Proposition 2.3, applied with $U=Z$ and $g$ as above.

We now prove some other versions of Proposition 5.1, by listing different hypotheses which give the same conclusion.

Proposition 5.2. Let $S$ be a finite nonabelian 2-group which satisfies at least one of the following conditions.

(a) There is an abelian subgroup $A \unlhd S$ of index two.

(b) $[S, S]$ is cyclic.

(c) There is an abelian subgroup $Q<S$ such that $\left|N_{S}(Q) / Q\right|=2$ and $\operatorname{Out}_{S}(Q) \not \leq$ $\mathrm{O}_{2}(\operatorname{Out}(Q))$.

(d) There is a subgroup $Q=Z(Q) U$ in $S$, where $U \cong Q_{8},\left|N_{S}(Q) / Q\right|=2$, and $\operatorname{Out}_{S}(Q)$ exchanges two of the three abelian subgroups of index two in $Q$.

(e) There is a subgroup $Q<S$ such that $|Q| \leq 16,\left|N_{S}(Q) / Q\right|=2$, and $\operatorname{Out}_{S}(Q) \not$ $\mathrm{O}_{2}(\operatorname{Out}(Q))$.

Then either $S$ is dihedral, semidihedral, or wreathed, or there is no reduced fusion system over $S$.

Proof. Assume there is a reduced fusion system $\mathcal{F}$ over $S$, and let $\mathcal{E}$ be the set of all $\mathcal{F}$ essential subgroups. Let $\mathcal{D S} \mathcal{W}$ be the class of all dihedral, semidihedral, and wreathed 2-groups; we must show $S \in \mathcal{D} \mathcal{S W}$.

(a) Let $A \leq S$ be an abelian subgroup of index two. If $R \in \mathcal{E}$ and $R \neq A$, then $R \not \leq A$ since $R$ is centric in $S$, and so $A R=S$. So there is $g \in A \cap N_{S}(R) \backslash R$, and $[g, R]$ is cyclic, generated by $[g, h]$ for any $h \in R \backslash A$. Hence by Lemma 1.5, $\left|N_{S}(R) / R\right|=2$. 
For each $x \in S \backslash A, C_{S}(x)=\left\langle C_{A}(x), x\right\rangle$ is abelian. Hence

$$
R \leq S, R \not \leq A C_{S}(R) \text { is abelian. }
$$

By Theorem 4.6(b,c), and since $[S, S] \leq A$ is abelian, each $R \in \mathcal{E}$ either has index two in $S$, or has the form described in Theorem 4.6(a) with $S_{*}=S$. In the latter case, there is $\Delta \unlhd S$ which is dihedral of order $\geq 8$ or quaternion of order $\geq 16$, and the noncyclic subgroups of index two in $\Delta$ are $S$-conjugate since $\Delta$ is the normal closure of a subgroup $U_{1} \cong C_{2}^{2}$ or $Q_{8}$. Also, for $\Delta_{0} \leq \Delta$ dihedral or quaternion of order $8, C_{S}\left(\Delta_{0}\right)$ is abelian by (1). The hypotheses of Proposition 5.1 thus hold, and so $S \in \mathcal{D S} \mathcal{W}$.

We are left with the case where each $R \in \mathcal{E}$ has index two in $S$. If all $\mathcal{F}$-essential subgroups are abelian, then $S$ has at least two abelian subgroups of index two (Lemma 2.2), so $|[S, S]|=2$ by Lemma A.6(c), and $S \in \mathcal{D} \mathcal{S W}$ by Proposition 2.5.

Assume $R \in \mathcal{E}$ is nonabelian. If $A \cap R$ is the only abelian subgroup of index two in $R$, then it is characteristic in $R$. For $g \in A \backslash R,[g, R] \leq R \cap A,[g, R \cap A]=1$, and this contradicts Lemma 1.4 (applied with $\Theta=R \cap A$ ).

Thus by Lemma A.6(b,c), $R$ has three abelian subgroups of index two, $[R: Z(R)]=4$, and $|[R, R]|=2$. Set $Z=[R, R] \leq Z(R)$, and fix a generator $z \in Z$. Fix $g \in A \backslash R$ and $h \in R \backslash A$, and set $y=[h, g]$. Thus $[g, R]=\langle y\rangle$, and

$$
h g h^{-1}=g y \Longrightarrow g=h^{2} g h^{-2}=g y\left(h y h^{-1}\right) \Longrightarrow[h, y]=h y h^{-1} y^{-1}=y^{-2} .
$$

If $y^{2}=1$, then $[h, y]=1$, so $[g, R]=\langle y\rangle \leq Z(R),[g, Z(R)]=1$ since $Z(R) \leq A$, and this again contradicts Lemma 1.4 (applied with $\Theta=Z(R)$ ). Thus $y^{2}=[h, y]^{-1} \neq 1$, so $y^{2}=z \in[R, R]$, and $[S, R]=\langle y,[R, R]\rangle=\langle y\rangle$ is cyclic.

By Lemma 2.4(a.ii) (and since $R$ is nonabelian), there are $R_{1} \unlhd R$ and $\operatorname{Out}_{S}(R) \leq$ $\Gamma_{1} \unlhd \operatorname{Out}_{\mathcal{F}}(R)$ such that $R_{1} \cong Q_{8}, \operatorname{Aut}_{\mathcal{F}}(R)$ sends $R_{1}$ to itself, and $\Gamma_{1} \cong \Sigma_{3}$ acts faithfully on $R_{1} / \operatorname{Fr}\left(R_{1}\right)$. Thus $R_{1} \unlhd S, \operatorname{Aut}_{S}(R)$ exchanges two of the three subgroups of index two in $R_{1}$, and $C_{S}\left(R_{1}\right)$ is abelian by (1). Hence $S \in \mathcal{D S} \mathcal{W}$ by Proposition 5.1.

(b) Assume $[S, S]$ is cyclic, and let $Z \leq[S, S]$ be the subgroup of order 2. If some $P \in \mathcal{E}$ has index $\geq 4$ in its normalizer, then $\operatorname{rk}([s, P / \operatorname{Fr}(P)]) \geq 2$ for each $s \in N_{S}(P) \backslash P$ by Lemma 1.5, which is impossible since $[S, S]$ is cyclic. If $P \in \mathcal{E}$ is nonabelian, then $1 \neq[P, P] \leq[S, S]$, and $Z=\Omega_{1}([P, P])$ is characteristic in $P$. So if each $P \in \mathcal{E}$ is nonabelian, then $Z$ is characteristic in $S$ and in each $P \in \mathcal{E}$, hence $Z \unlhd \mathcal{F}$ by Proposition 1.3 , and $\mathcal{F}$ is not reduced.

Thus some $R \in \mathcal{E}$ is abelian and has index 2 in its normalizer. If $[S: R]=2$, then the result follows from (a). Otherwise, by Theorem 4.6, there is $\Delta \unlhd S$ which is dihedral of order $\geq 8$ ( $R \geq Q_{8}$ since it is abelian), and the noncyclic subgroups of index two in $\Delta$ are $S$-conjugate since $\Delta$ is the normal closure of a subgroup $U_{1} \cong C_{2}^{2}$ in $R$. Also, for $\Delta_{0} \leq \Delta$ dihedral of order 8 containing $U_{1}, C_{S}\left(\Delta_{0}\right) \leq C_{S}\left(U_{1}\right) \leq R$ is abelian. The hypotheses of Proposition 5.1 thus hold, and so $S \in \mathcal{D} \mathcal{S W}$.

(c) If $S$ contains an abelian subgroup of index two, then $S \in \mathcal{D S} \mathcal{W}$ by (a). If there is an abelian subgroup $Q<S$ which satisfies the assumptions in (c) and is not normal, then by Lemma B.5(a), there are subgroups $\Delta_{0} \leq \Delta \unlhd S$ such that $\Delta$ is dihedral, $\Delta_{0} \cong D_{8}, C_{S}\left(\Delta_{0}\right) \leq Q$ is abelian, and the noncentral involutions in $\Delta$ are all $S$-conjugate. Then $S \in \mathcal{D} \mathcal{S} \mathcal{W}$ by Proposition 5.1.

(d) Fix $Q=Z(Q) U \leq S$, where $U \cong Q_{8},\left|N_{S}(Q) / Q\right|=2$, and $\operatorname{Out}_{S}(Q)$ exchanges two abelian subgroups $A_{1}$ and $A_{2}$ of index two in $Q$. Set $Z=Z(U)=[Q, Q] \cong C_{2}$, let $z \in Z$ be the generator, choose $u_{1} \in A_{1} \cap U$ of order 4 , and set $u_{2}=x u_{1} x^{-1} \in A_{2}$ for 
some $x \in N_{S}(Q) \backslash Q$. Then $u_{2}^{2}=u_{1}^{2}=z$ since $[x, z]=1, u_{2} \notin Z(Q)$, and so $\left[u_{1}, u_{2}\right]=z$ and $\left\langle u_{1}, u_{2}\right\rangle \cong Q_{8}$. So upon replacing $U$ by $\left\langle u_{1}, u_{2}\right\rangle$, we can assume $U \unlhd N_{S}(Q)$.

Set $S_{*}=C_{S}(Z)$, and let $\Delta$ be the normal closure of $U$ in $S_{*}$. Then $Q / Z$ is abelian, and $\left[N_{S_{*} / Z}(Q / Z): Q / Z\right]=2$ since $\left[N_{S}(Q): Q\right]=2$ and $N_{S_{*}}(Q)=N_{S}(Q)$. Also, $U / Z \cong$ $C_{2}^{2}$ is a direct factor in $Q / Z$, and $\left[N_{S}(Q) / Z, U / Z\right] \neq 1$ since $\operatorname{Out}_{S}(Q)$ exchanges two of the abelian subgroups of $U$.

If $\Delta>U$, then $\Delta / Z$ is dihedral by Lemma B.5(b), and all involutions in $\Delta / Z$ are $S_{*}$-conjugate to elements of $U / Z$. Hence there are no involutions in $\Delta \backslash Z$, and $\Delta$ is quaternion.

Set $R=N_{C_{S}(U)}(Z(Q))$. Since conjugation by each $y \in N_{S}(Q) \backslash Q$ exchanges $A_{1}$ and $A_{2}, C_{S}(U) \cap N_{S}(Q) \leq Q$. Also, $R$ normalizes $Z(Q) U=Q$, so $R \leq C_{S}(U) \cap N_{S}(Q) \leq Q$. Hence $R \leq C_{Q}(U)=Z(Q)$, so $C_{S}(U)=Z(Q)$ by Lemma A.1.

If $S_{*}<S$, then choose $y \in N_{S}\left(S_{*}\right) \backslash S_{*}$, and set $\beta=c_{y} \in \operatorname{Aut}\left(S_{*}\right)$. Thus $\beta(Z) \neq Z$, and $\Delta \cap \beta(\Delta)=1$ since $Z$ and $\beta(Z)$ contain the only elements of order two in $\Delta$ and $\beta(\Delta)$, respectively. So $[\Delta, \beta(\Delta)] \leq \Delta \cap \beta(\Delta)=1$ since both subgroups are normal, which is impossible since $C_{S}(\Delta) \leq C_{S}(U)=Z(Q)$ is abelian. Thus $S_{*}=S$.

If $\Delta=U$, then by assumption, $\operatorname{Aut}_{S}(Q)$ exchanges two of the abelian subgroups of index two in $Q=Z(Q) U$, and hence exchanges two of the index two subgroups of $U=\Delta$. If $\Delta>U$, the two noncyclic subgroups of index two in $\Delta$ are $S$-conjugate since $\Delta$ is generated by the $S$-conjugates of $U<\Delta$. Since $C_{S}(U)=Z(Q)$ is abelian, $S \in \mathcal{D S} \mathcal{W}$ by Proposition 5.1, applied with $\Delta_{0}=U$.

(e) Assume $Q<S$ is such that $|Q| \leq 16,\left|N_{S}(Q) / Q\right|=2$, and $\operatorname{Out}_{S}(Q) \not \leq O_{2}(\operatorname{Out}(Q))$. In particular, Out $(Q)$ is not a 2-group. By Corollary A.3(a,c), $Q^{\text {ab }} \neq C_{4} \times C_{2}$, so either $Q$ is abelian or $Q^{\text {ab }}$ is elementary abelian. If $Q$ is abelian, then $S \in \mathcal{D S} \mathcal{W}$ by (c). If $Q$ is nonabelian, then by Corollary A.3(b) and the list of groups of order 16 (cf. [Bu, $\S 74]$ ), $Q$ is isomorphic to $Q_{8}, C_{2} \times Q_{8}$, or $C_{4} \times C_{2} Q_{8}$. (Note that $\operatorname{Aut}\left(C_{2} \times D_{8}\right)$ is a 2-group by Lemma A.2, since it contains a unique subgroup isomorphic to $C_{2} \times C_{4}$.) In each of these cases, by Lemma A.2 applied to the chain $1<\operatorname{Fr}(Q) \leq Z(Q)<Q, O_{2}(\operatorname{Aut}(Q))$ contains all $\alpha \in \operatorname{Aut}(Q)$ which act trivially on $Q / Z(Q)$. Since $\operatorname{Aut}_{S}(Q) \not \leq O_{2}(\operatorname{Aut}(Q))$, $\operatorname{Aut}_{S}(Q)$ acts nontrivially on $Q / Z(Q)$ and hence contains elements which exchange two of the three abelian subgroups of index two in $Q$. So $S \in \mathcal{D S} \mathcal{W}$ by (d).

Each reduced fusion system over a dihedral, semidihedral, or wreathed 2-group is isomorphic to the fusion system of $P S L_{2}(q)$ for $q \equiv \pm 1(\bmod 8)$, or of $P S U_{3}(q)$ or $P S L_{3}(q)$ for $q \equiv 1(\bmod 4)$. Fusion systems over dihedral and semidihedral 2-groups have been listed by several people; cf. [AOV, §4.1] for the reduced case. For wreathed 2-groups, this was shown in Proposition 3.1. The fusion systems (at the prime 2) of $P S U_{3}(q)$ and $P S L_{3}(q)$ for $q \equiv 3(\bmod 4)$ also have this form (see, e.g., [BMO, Theorem $\mathrm{A}(\mathrm{d})])$.

Proposition 5.2(e) has as an easy consequence:

Theorem 5.3. Let $\mathcal{F}$ be a reduced fusion system over a nontrivial finite 2-group $S$ of order at most 32. Then $S$ is dihedral, semidihedral, or wreathed, and $\mathcal{F}$ is isomorphic to the fusion system of $P S L_{2}(q)$ for $q \equiv \pm 1(\bmod 8)$, or that of $P S L_{3}(q)$ for $q$ odd.

Proof. By Lemma 2.2, there exists an $\mathcal{F}$-essential subgroup $Q<S$. Then $|Q| \leq 16$, and $\left|N_{S}(Q) / Q\right|=2$, since otherwise $|S| \geq\left|N_{S}(Q)\right| \geq 64$ by Lemma 1.5. Since $\operatorname{Out}_{\mathcal{F}}(Q)$ contains a strongly 2-embedded subgroup, $\mathrm{O}_{2}\left(\operatorname{Out}_{\mathcal{F}}(Q)\right)=1$ (cf. [AKO, Proposition 
A.7(c)]). Hence $\operatorname{Out}_{S}(Q) \not \leq O_{2}(\operatorname{Out}(Q))$, so $S$ is dihedral, semidihedral or wreathed by Theorem $5.2(\mathrm{e})$. The description of $\mathcal{F}$ follows from the above remarks.

We finish with a slightly less easy consequence of the results in this section and in Section 4: a list of those groups of order 64 which support reduced fusion systems. For all $n$ and $q, U T_{n}(q)$ denotes the group of strictly upper triangular matrices over $\mathbb{F}_{q}$ (i.e., those with 1's on the diagonal). A fusion system over a $p$-group $S$ is indecomposable if it is not isomorphic to a product of fusion systems over nontrivial subgroups of $S$.

Theorem 5.4. Let $\mathcal{F}$ be a reduced, indecomposable fusion system over $S$, where $|S|=$ 64. Then $S$ is isomorphic to one of the groups $D_{64}, S D_{64}, U T_{4}(2), U T_{3}(4)$, or to a Sylow 2-subgroup of $M_{12}$.

Proof. Fix $\mathcal{F}$ and $S$, and let $\mathcal{E}$ be the set of $\mathcal{F}$-essential subgroups of $S$. Assume $S$ is neither dihedral nor semidihedral; it cannot be wreathed since $|S|$ is a power of 4 .

Case 1: Assume there is $P \in \mathcal{E}$ such that $P \not S$ and $\left|N_{S}(P) / P\right|=2$. Then $|P| \leq 16$, $\operatorname{Out}_{S}(P) \not \leq O_{2}(\operatorname{Out}(P))$ since $P$ is essential, and this is impossible by Proposition $5.2(\mathrm{e})$.

Case 2: Assume that we are not in the situation of Case 1, and that there is $P \in \mathcal{E}$ such that $\left|N_{S}(P) / P\right| \geq 4$. By Lemma $1.5, \operatorname{rk}(P / \operatorname{Fr}(P)) \geq 4$. Thus $P \cong C_{2}^{4}$ and $P \unlhd S$. Using Bender's classification of groups with strongly 2-embedded subgroups [Bd, Satz 1], we see that either $\operatorname{Aut}_{\mathcal{F}}(P) \geq A_{5}$, or $\operatorname{Aut}_{\mathcal{F}}(P) \cong C_{5} \rtimes C_{4}, C_{15} \rtimes C_{4}$, or $C_{3}^{2} \rtimes C_{4}$. Also, there are exactly two conjugacy classes of subgroups isomorphic to $A_{5}$ in $G L_{4}(2) \cong A_{8}$ (cf. [Ta, Corollary 6.7]), corresponding to the $A_{5}$-orbits $A_{5} / A_{4}$ and $A_{5} / D_{10}$.

Case 2A: Assume $A_{5} \leq \operatorname{Aut}_{\mathcal{F}}(P)$, acting via the reduced permutation action (i.e., the permutation action on $\mathbb{F}_{2}^{5}$ modulo its fixed subspace). Then $\operatorname{Aut}_{S}(P) \cong C_{2}^{2}$ permutes freely a basis for $P \cong C_{2}^{4}$, so the extension of $P$ by $S / P$ splits, and $S \cong U T_{4}(2)$.

Case 2B: Assume $A_{5} \leq \operatorname{Aut}_{\mathcal{F}}(P)$, acting via the canonical action of $A_{5} \cong S L_{2}(4)$ on $\mathbb{F}_{4}^{2}$. With respect to a suitable basis, each $g \in S \backslash P$ acts via multiplication by a triangular matrix $\left(\begin{array}{ll}1 & x \\ 0 & 1\end{array}\right)$ for some $x \in \mathbb{F}_{4} \backslash\{0\}$, so $C_{P}(g)=[g, P]=Z(S) \cong C_{2}^{2}$. Thus $g^{2} \in C_{P}(g)=Z(S)$ for each $g \in S$, so $S / Z(S)$ has exponent two and hence is abelian. It follows that $[S, S]=Z(S)$, and in particular, that each $R \in \mathcal{E}$ is normal in $S$.

Assume $R \in \mathcal{E}$ with $[S: R]=2$. If $P \leq R$, then $[g, P]=[S, S]$ for any $g \in R \backslash P$. If $P \not \leq R$, then $S=P R, \operatorname{rk}(P \cap R)=3>\operatorname{rk}(Z(S))$, and for $g \in(P \cap R) \backslash Z(S)$, $[R, R] \geq[R, g]=[P R, g]=\left[\operatorname{Aut}_{S}(P), g\right]=[S, S]$. Thus $[R, R]=[S, S]$ in both cases. Hence $[S, R]=[R, R] \leq \operatorname{Fr}(R)$, which contradicts Lemma 1.4 (applied with $\Theta=1$ ).

By Lemma 2.2 , there is $R \in \mathcal{E}$ with $R \neq P$, and we just showed that $[S: R]>2$. Hence $\left|N_{S}(R) / R\right| \geq 4$, so $R \cong C_{2}^{4}$ by Lemma 1.5. Also, $Z(R P)=Z(S) \cong C_{2}^{2}$ since $C_{P}(g)=Z(S)$ for each $g \in S \backslash P$, so $|R \cap P| \leq|Z(R P)|=4$, and $R P=S$. In particular, the extension of $P$ by $S / P \cong C_{2}^{2}$ is split, and so $S \cong U T_{3}(4)$.

Case 2C: Assume $\operatorname{Aut}_{S}(P) \cong S / P \cong C_{4}$, and thus $\operatorname{Aut}_{\mathcal{F}}(P) \cong C_{5} \rtimes C_{4}, C_{15} \rtimes C_{4}$, or $C_{3}^{2} \rtimes C_{4}$. In either case, there is a basis of $P \cong C_{2}^{4}$ permuted freely by $S / P \cong C_{4}$, and so $C_{P}(S) \leq[S, P] \cong C_{2}^{3}$. If $x \in S$ is such that $S=\langle P, x\rangle$, then $x^{4} \in C_{P}(S) \leq[S, P]$, and so $[S, S]=[S, P]$ and $S /[S, S] \cong C_{2} \times C_{4}$.

Let $Q=\left\langle P, x^{2}\right\rangle<S$ be the (unique) subgroup of index two which contains $P$. Thus $Q /[S, S] \cong C_{2}^{2}$, and in particular, each elementary abelian subgroup of $S$ is contained in $Q$. 
For each $R \in \mathcal{E}$, we have shown that either $[S: R]=2$ or $R \cong C_{2}^{4}$. If $R \cong C_{2}^{4}$, then $R \leq Q$, and $R P=Q$ if $R \neq P$. (In fact, there is no such subgroup.) If [S:R]=2 and $R \neq Q$, then $R \cap P \cong C_{2}^{3}$, so $R \cap P=[S, P]$ since it is normal in $S, R P=S$, and $[R, R]=[S, R \cap P]$ has index two in $R \cap P$. So $R /[R, R] \cong C_{2} \times C_{4}$, Aut $(R)$ is a 2-group by Corollary A.3(a,c), and $R \notin \mathcal{E}$. Thus $R P=Q$ for each $R \in \mathcal{E} \backslash\{P\}$. So $\mathcal{E}=\{P\}$ or $\langle\mathcal{E}\rangle=Q$, either of which contradicts Lemma 2.2. Hence this case is impossible.

Case 3: We are left with the case where each $\mathcal{F}$-essential subgroup has index two in $S$. Since $|\mathcal{E}| \geq 2$ by Lemma 2.2 , we can choose distinct $P_{1}, P_{2} \in \mathcal{E}$. By Theorem 4.5, we can choose $G_{1}>S<G_{2}$ and $T \leq P_{12}=P_{1} \cap P_{2}$ which satisfy conditions (*) at the beginning of Section 4, with $P=S$ and $\operatorname{Aut}_{G_{i}}\left(P_{i}\right) \leq \operatorname{Aut}_{\mathcal{F}}\left(P_{i}\right)$. Thus $\operatorname{Aut}_{G_{i}}(T) \leq \operatorname{Aut}_{\mathcal{F}}(T)$.

Case 3A: Assume $T$ is not centric in $S$. Let $U$ and $Z=T \cap U$ be as in Theorem 4.5; $[T, U]=1$ in all cases. There are six cases to consider, listed in Table 4.1.

(1) $|T|=8$, and $S \cong T \times D_{8}$ or $T \times_{C_{2}} Q_{16}$. The first is impossible by [O1, Theorem B] (and since $\mathcal{F}$ is indecomposable), and the second by Proposition 5.2(b) $([S, S]=$ $[U, U]$ is cyclic).

(2) $|T|=4,[S: U T]=2, U \unlhd S,(U, Z) \cong\left(D_{8}, 1\right)$ or $\left(Q_{16}, C_{2}\right)$, and $S / T \cong C_{2} \times D_{8}$. Then $S=\langle U T, x\rangle$ for some $x$ such that $x T \in Z(S / T)$. If $U \cong D_{8}$, then $S=$ $U \times\langle T, x\rangle$, which is impossible by [O1, Theorem B] again. Hence $U \cong Q_{16}$, $[x, U] \leq T \cap U=Z$ since $[x, S] \leq T$ and $U \unlhd S$, and $[x, T] \leq Z$ since $[T: Z]=2$ and $Z \unlhd S$. Thus $[S, S]=[U, U]$ is cyclic, and this is impossible by Proposition $5.2(\mathrm{~b})$.

(3) $U \cong C_{4} \prec C_{2}, Z=1$, and $S=U T$. Then $S \cong U \times C_{2}$ has an abelian subgroup of index two, which is impossible by Proposition 5.2(a).

(4) $U \cong C_{4} \succ C_{2}, Z=1$, and $[S: U T]=2$. Thus $T=1$, and $S$ is of type $\operatorname{Aut}\left(U_{3}(3)\right)$. By the descriptions of this amalgam in [Gd2, Table $1 \&(3.7)], S$ is isomorphic to a Sylow 2-subgroup of $M_{12}$.

(5) $S=U$ is a Sylow subgroup of $M_{12}$.

(6) $|U| \geq 2^{7}$, so this is impossible.

In the remaining cases, we assume $T$ is centric in $S$. Set $\widehat{S}=\operatorname{Out}_{S}(T), \widehat{G}_{i}=$ $\operatorname{Out}_{G_{i}}(T), \widehat{G}=\left\langle\widehat{G}_{1}, \widehat{G}_{2}\right\rangle \leq \operatorname{Out}_{\mathcal{F}}(T)$, and $\widehat{P}_{i}=\operatorname{Out}_{P_{i}}(T)$. Thus $\widehat{S} \in \operatorname{Syl}_{2}(\widehat{G})$ by the Sylow axiom since $T \unlhd S$ (hence $T$ is fully normalized). Let $\Gamma$ be a model for $T \unlhd N_{\mathcal{F}}(T)$ (cf. [AKO, Theorem III.5.10(a)]), and let $G \leq \Gamma$ be such that $T \leq G$ and $\operatorname{Out}_{G}(T)=\widehat{G}$. Thus $G$ is a finite group such that

$$
S \in \operatorname{Syl}_{2}(G), \quad T \unlhd G, \quad C_{G}(T) \leq T, \quad \text { and } \quad \widehat{G}=\operatorname{Out}_{G}(T) .
$$

Case 3B: Assume $[S: T]=4$. Thus $\widehat{S} \cong S / T \cong C_{2}^{2}$ by $(*), T=P_{12}, P_{i} / T \cong C_{2}$ is normal in $\widehat{G}_{i} \cong G_{i} / T$ with quotient group $G_{i} / P_{i} \cong D_{2 p_{i}}$, and hence $\widehat{G}_{i} \cong C_{2} \times D_{2 p_{i}}$ contains an element of order $2 p_{i}$. Also, $\widehat{G}$ acts faithfully on $T / \operatorname{Fr}(T)$ by Lemma $4.2(\mathrm{e})$, $G L_{4}(2) \cong A_{8}$ contains no element of order $2 p_{i}$ for primes $p_{i} \geq 5$, and $G L_{3}(2)$ contains no element of order 6 . It follows that $\operatorname{rk}(T / \operatorname{Fr}(T))=4$, so $T \cong C_{2}^{4}$, and $p_{1}=p_{2}=3$.

Set $\widehat{H}_{i}=O^{2}\left(\widehat{G}_{i}\right) \cong C_{3}$. It is not hard to see, by examining normalizers of subgroups of order three in $G L_{4}(2) \cong A_{8}$, that there are exactly two conjugacy classes of subgroups $C_{2} \times \Sigma_{3}$ in $G L_{4}(2)$. Hence for some decomposition $T=V_{1} \times V_{2}$, where $V_{i} \cong C_{2}^{2}$, the action of $\widehat{G}_{1} \cong C_{2} \times \Sigma_{3}$ on $T$ has the following form: either 
(i) $\Sigma_{3}$ acts faithfully on each $V_{i}$, and $C_{2}$ switches the two factors; or

(ii) $\Sigma_{3}$ acts faithfully on $V_{1}$ and trivially on $V_{2}$, and $C_{2}$ acts faithfully on $V_{2}$ and trivially on $V_{1}$.

In case (i), $\widehat{S}$ acts by permuting freely a basis of $T$, and hence $S \cong U T_{4}(2)$ as seen earlier.

In case (ii), there is a basis of $T$ permuted by $\widehat{S}$ in two orbits of length 2 . Hence the three involutions in $\widehat{S}$ cannot be conjugate in $\widehat{G}$, so $\mathcal{F}_{\widehat{S}}(\widehat{G})$ is the fusion system of $\widehat{S} \cong C_{2}^{2}$. Thus $[\widehat{G}, \widehat{G}] \cap \widehat{S}=1$ by the focal subgroup theorem (cf. [G, Theorem 7.3.4]), so there is $\widehat{H} \unlhd \widehat{G}$ of odd order and index four. Also, $\widehat{P}_{i} \unlhd \widehat{G}_{i}$ for $i=1,2$, where $\widehat{P}_{i} \cong C_{2}$ and $\widehat{G}_{i} \cong C_{2} \times \Sigma_{3}$, so $\widehat{G}_{1} \neq \widehat{G}_{2}$. Thus $\widehat{G}=\left\langle\widehat{G}_{1}, \widehat{G}_{2}\right\rangle$ contains two distinct subgroups of order 3 , they are contained in $\widehat{H}$, and hence $|\widehat{H}|>3$. Also, $\widehat{H}$ cannot contain a normal subgroup $\widehat{K} \unlhd \widehat{H}$ of order 7 , since that would imply $\widehat{G} \leq N_{\operatorname{Aut}(T)}(\widehat{K}) \cong C_{7} \rtimes C_{3}$. Since $\left|G L_{4}(2)\right|=2^{6} \cdot 3^{2} \cdot 5 \cdot 7, \widehat{H}$ contains $C_{3}^{2}$ or $C_{5}$, and in either case, $C_{T}(\widehat{H})=1$. Let $H<G$ be such that $H \geq T$ and $\operatorname{Aut}_{H}(T)=\widehat{H}$. By Lemma A.9, applied to the triple $T \unlhd H \unlhd G, G$ (and hence $S$ ) splits as a semidirect product over $T$. Thus $S \cong D_{8} \times D_{8}$, which is impossible by [O1, Theorem B] again $(\mathcal{F}$ is indecomposable).

Case 3C: Now assume $[S: T] \geq 8$. Since $\widehat{S}$ acts faithfully on $T / \operatorname{Fr}(T), \operatorname{rk}(T / \operatorname{Fr}(T)) \geq$ 3 , so $T \cong C_{2}^{3},|\widehat{S}|=|S / T|=8$, and $\widehat{S} \cong D_{8}$ since this is a Sylow 2-subgroup of $G L_{3}(2)$. Neither $\widehat{P}_{1}$ nor $\widehat{P}_{2}$ can be cyclic of order four, since the normalizer in $\operatorname{Aut}(T) \cong G L_{3}(2)$ of such a subgroup has order 8. Hence $\widehat{P}_{i} \cong C_{2}^{2}$ for $i=1,2, \widehat{G}_{i} \cong \Sigma_{4}$ (the normalizer in $\operatorname{Aut}(T) \cong G L_{3}(2)$ of $\left.\widehat{P}_{i}\right)$, and $\widehat{G}=\left\langle\widehat{G}_{1}, \widehat{G}_{2}\right\rangle=\operatorname{Aut}(T)$. Thus $G$ is an extension of $C_{2}^{3}$ by $G L_{3}(2)$. By [GH, Lemma II.3.4], either the extension is split and $S \cong U T_{4}(2)$, or it is not split and $S$ is a Sylow 2-subgroup of $M_{12}$.

\section{APPENDIX A. BACKGROUND ON GROUPS}

We list here some elementary results about finite groups which are needed throughout the paper, beginning with a well known property of $p$-groups.

Lemma A.1. If $Q<P$ are finite p-groups for some prime $p$, then $Q<N_{P}(Q)$.

Proof. See, e.g., [Sz1, Theorem 2.1.6].

We next look at automorphisms of finite $p$-groups.

Lemma A.2. Fix a prime $p$, a finite $p$-group $P$, a subgroup $P_{0} \leq \operatorname{Fr}(P)$, and a sequence of subgroups

$$
P_{0} \unlhd P_{1} \unlhd \cdots \unlhd P_{k}=P
$$

Set

$$
\mathcal{A}=\left\{\alpha \in \operatorname{Aut}(P) \mid x^{-1} \alpha(x) \in P_{i-1}, \text { all } x \in P_{i} \text {, all } i=1, \ldots, k\right\} \leq \operatorname{Aut}(P):
$$

the group of automorphisms which leave each $P_{i}$ invariant, and which induce the identity on each quotient group $P_{i} / P_{i-1}$. Then $\mathcal{A}$ is a p-group. If the $P_{i}$ are all characteristic in $P$, then $\mathcal{A} \unlhd \operatorname{Aut}(P)$, and hence $\mathcal{A} \leq O_{p}(\operatorname{Aut}(P))$.

Proof. See, for example, [G, Theorems 5.1.4 \& 5.3.2]. 
As an easy exercise, Lemma A.2 implies the following corollary, which contains a list of 2-groups whose automorphism groups are 2-groups. Recall that $G^{\mathrm{ab}}=G /[G, G]$ denotes the abelianization of a group $G$.

Corollary A.3. For a finite 2-group $P, \operatorname{Aut}(P)$ is a 2-group if at least one of the following hold:

(a) $P \cong C_{2^{k_{1}}} \times C_{2^{k_{2}}} \times \cdots \times C_{2^{k_{r}}}$, where $k_{1}, \ldots, k_{r}$ are pairwise distinct.

(b) $P$ is dihedral of order $\geq 8$, or semidihedral or quaternion of order $\geq 16$.

(c) $\operatorname{Aut}\left(P^{\mathrm{ab}}\right)$ is a 2-group.

The next two results involve the intersection of a Sylow subgroup with the commutator subgroup.

Lemma A.4. For any finite group $G$ with $S \in \operatorname{Syl}_{p}(G), S \cap O^{p}(G) \leq S \cap[G, G]$, with equality if $G / O^{p}(G)$ is abelian.

Proof. Set $G^{\prime}=[G, G]$. Since $G / G^{\prime}$ is abelian, its largest $p$-group quotient $G / O^{p}(G) G^{\prime}$ is isomorphic to its Sylow $p$-subgroup $S G^{\prime} / G^{\prime} \cong S /\left(S \cap G^{\prime}\right)$ (and the isomorphism is induced by the inclusion $S \leq G)$. Hence $S \cap G^{\prime}=S \cap O^{p}(G) G^{\prime}$, and so $S \cap O^{p}(G) \leq$ $S \cap G^{\prime}$ with equality if $G^{\prime} \leq O^{p}(G)$.

The following proposition goes essentially back to Schur [Sch, IX-X].

Proposition A.5. Fix a finite group $G$ with $S \in \operatorname{Syl}_{p}(G)$. Let $Z \leq Z(G)$ be a psubgroup. Then $Z \cap[G, G]=Z \cap[S, S]$.

Proof. This follows as an application of the transfer in (co)homology. See, e.g., [Hu, Satz IV.2.2].

The next lemma describes nonabelian 2-groups with abelian subgroup of index two.

Lemma A.6. Let $S$ be a finite nonabelian 2-group containing an abelian subgroup $A \unlhd S$ of index two. Then the following hold.

(a) $[S, S] \cong A / Z(S)$, and all elements of $(S / Z(S)) \backslash(A / Z(S))$ have order two.

(b) If $|[S, S]|=2$, then $S / Z(S) \cong C_{2}^{2}$, and $S$ contains exactly three abelian subgroups of index two.

(c) If $|[S, S]| \geq 4$, then $|S / Z(S)| \geq 8$, and $A$ is the unique abelian subgroup of index two in $S$.

(d) If $S$ contains three abelian subgroups of index two which are permuted transitively by some automorphism of $S$, then either $Z(S)$ is not a direct factor of $A$, or $[S, S] \leq \operatorname{Fr}(Z(S))$.

Proof. (a) For each $x \in S \backslash A, x^{2} \in C_{A}(x)=Z(S)$, and thus $x Z(S)$ has order two in $S / Z(S)$. Also, $[S, S]=[x, A]$ is the image of $\mathrm{Id}-c_{x}$ as a homomorphism from $A$ to itself, and $Z(S)$ is its kernel. Hence $[S, S] \cong A / Z(S)$.

(b) Assume $|[S, S]|=2$. Then by (a), $|S / Z(S)|=4$ and $S / Z(S) \cong C_{2}^{2}$. Each abelian subgroup of index two contains $Z(S)$, and each subgroup of index two which contains $Z(S)$ is abelian. So there are exactly three abelian subgroups of index two in $S$. 
(c) Now assume $|[S, S]| \geq 4$, so $|S / Z(S)| \geq 8$ by (a). If $B \neq A$ is another abelian subgroup of index two in $S$, then $A B=S$, so $Z(S) \geq A \cap B$, and $|S| Z(S) \mid \leq 4$, a contradiction. Thus $A$ is the only abelian subgroup of index two.

(d) Assume $S$ contains three abelian subgroups $A=A_{1}, A_{2}, A_{3}$ of index two which are permuted transitively by some automorphism of $S$. Thus $|[S, S]|=2$ and $S / Z(S) \cong C_{2}^{2}$ by $(\mathrm{b}, \mathrm{c})$. Fix a generator $z \in[S, S]$. If $Z(S)$ is a direct factor of $A=A_{1}$, then it is a direct factor of each $A_{i}$, and there are elements $a_{i} \in A_{i} \backslash Z(S)$ of order 2. Then $a_{1} a_{2} a_{3} \in Z(S)$ since the $a_{i}$ represent the three nonidentity elements in $S / Z(S) \cong C_{2}^{2}$, $\left[a_{i}, a_{j}\right]=z$ for distinct $i, j \in\{1,2,3\}$ since $S=\left\langle a_{i}, a_{j}, Z(S)\right\rangle$ is nonabelian, and so $\left(a_{1} a_{2} a_{3}\right)^{2}=z^{3}=z$. Thus $z \in \operatorname{Fr}(Z(S))$.

Recall that a group $G$ is metacyclic if it contains a normal cyclic subgroup $H \unlhd G$ such that $G / H$ is also cyclic.

Lemma A.7. Let $S$ be a finite metacyclic 2-group with an automorphism $\gamma \neq \mathrm{Id}_{S}$ of odd order. Then either $S \cong C_{2^{n}} \times C_{2^{n}}$ for some $n \geq 1$ and $C_{S}(\gamma)=1$, or $S \cong Q_{8}$.

Proof. If $S$ is abelian, then $\operatorname{rk}(S) \leq 2$, and $S \cong C_{2^{n}} \times C_{2^{n}}$ by Corollary A.3(a) (and since $\operatorname{Aut}(S)$ is not a 2 -group). Also, $C_{S}(\gamma)=1$ since $\gamma$ acts nontrivially on $\Omega_{1}(S) \cong C_{2}^{2}$ (see [G, Theorem 5.2.4]).

Now assume $S$ is nonabelian. Fix $A=\langle a\rangle \unlhd S$ such that $S / A$ is cyclic, and let $b \in S$ be such that $S=\langle a, b\rangle$. Set $S^{\prime}=[S, S] \leq A$. Then $\left.\gamma\right|_{S^{\prime}}=$ Id since $S^{\prime} \leq A$ is cyclic. Let $\bar{\gamma} \in \operatorname{Aut}\left(S / S^{\prime}\right)$ be the automorphism induced by $\gamma$. Then $\bar{\gamma} \neq \operatorname{Id}_{S / S^{\prime}}$ by Lemma A.2, so $\bar{\gamma}$ fixes only the identity element by the first paragraph, and $C_{S}(\gamma)=S^{\prime}$. Also, $Z(S)$ is abelian of rank $\leq 2$ and $\left.\gamma\right|_{Z(S)}$ has nonidentity fixed points (since $S^{\prime} \cap Z(S) \neq 1$ ), so $\left.\gamma\right|_{Z(S)}=$ Id by the first paragraph, and $Z(S) \leq S^{\prime}$. Since $\gamma$ does not induce the identity on $S / \operatorname{Fr}(S) \cong C_{2}^{2}$ (and $\left.a \notin \operatorname{Fr}(S)\right), S=\langle a, \gamma(a), \operatorname{Fr}(S)\rangle$, so $S=\langle a, \gamma(a)\rangle$ since $\operatorname{Fr}(S)$ is the intersection of all maximal subgroups. Thus $\left[S^{\prime}, S\right]=1$, and $S^{\prime}=Z(S)$. Also, $\left[a^{i}, b^{j}\right]=[a, b]^{i j}$ for all $i, j \geq 1$ since $[a, b] \in Z(S)$.

Set $S_{0}=\operatorname{Fr}(S)=\left\langle a^{2}, b^{2}\right\rangle \geq S^{\prime}$ (since $[a, b] \in\left\langle a^{2}\right\rangle$ ). If $S_{0}>S^{\prime}$, then $\left.\gamma\right|_{S_{0}} \neq$ Id and $C_{S_{0}}(\gamma) \neq 1$. Hence $S_{0}$ is nonabelian, and we just showed this implies $Z\left(S_{0}\right)=\left[S_{0}, S_{0}\right]$. But $\left[S_{0}, S_{0}\right]=\left\langle\left[a^{2}, b^{2}\right]\right\rangle=\left\langle[a, b]^{4}\right\rangle<S^{\prime} \leq Z\left(S_{0}\right)$, so this is impossible. We conclude that $S_{0}=S^{\prime}$, and hence is generated by $[a, b]$. Also, $b^{2} \in A$ implies $[a, b]^{2}=\left[a, b^{2}\right]=1$, so $\left|S^{\prime}\right|=2,|S|=8$, and $S \cong Q_{8}$.

The following result about actions on abelian 2-groups is very useful in certain situations.

Lemma A.8. Fix a finite abelian 2-group $A$ and a subgroup $G \leq \operatorname{Aut}(A)$ with Sylow subgroup $S \in \operatorname{Syl}_{2}(G)$ of order two. Assume $S \not Z Z(G)$, and $[S, A] \cong C_{2^{n}}$ for some $n \geq 1$. Then there are unique decompositions $A=A_{0} \times A_{1}$ and $G=G_{0} \times G_{1}$, such that the $G$-action on $A$ splits as a product of $G_{i}$-actions on $A_{i}$; and such that $\left|G_{0}\right|$ is odd, $G_{1} \cong \Sigma_{3}$, and $A_{1} \cong C_{2^{n}} \times C_{2^{n}}$.

Proof. See, e.g., [O1, Proposition 2.3].

Lemma A.9. Fix a prime $p$, and a finite group $G$ with subgroups $A \unlhd B \unlhd G$, both normal in $G$, such that $A$ is an abelian p-group, $B / A$ has order prime to $p$, and $C_{A}(B)=1$. Then $G$ splits as a semidirect product $G=A \rtimes H$, where $H \cong G / A$. 
Proof. By the spectral sequence for the extension $1 \rightarrow B / A \rightarrow G / A \rightarrow G / B \rightarrow$ $1, H^{i}(G / A ; A)=0$ for each $i \geq 0$ since $H^{0}(B / A ; A)=C_{A}(B)=0$ (and since $(|B / A|,|A|)=1)$. In particular, $G \cong A \rtimes(G / A)$.

Alternatively, since $|B / A|$ is prime to $|A|$, by the Schur-Zassenhaus theorem $[\mathrm{G}$, Theorem 6.2.1], there is $K \leq B$ such that $K A=B$ and $K \cap A=1$, and each such subgroup is $B$-conjugate to $K$. Hence the $B$-conjugacy class of $K$ is its $G$-conjugacy class, so $\left[N_{G}(K): N_{B}(K)\right]=[G: B]$. If $a \in A \cap N_{G}(K)$, then $[a, K] \leq A \cap K=1$, so $a=1$ since $C_{A}(K)=C_{A}(B)=1$. Thus $N_{G}(K) \cap A=1$, so $N_{B}(K)=K$, and $G=A N_{G}(K)$.

\section{ApPendix B. Finite 2-GRoups With NORMAL DIHEDRAL OR QUATERNION SUBGROUPS}

We prove here some elementary results about certain finite 2-groups which have normal dihedral or quaternion subgroups, and their automorphisms. We begin by stating the following general proposition about automorphisms of products of $p$-groups.

Proposition B.1 ([O1, Proposition 3.2(a)]). Fix a pair of finite p-groups $S_{1}$ and $S_{2}$, set $S=S_{1} \times S_{2}$, and let $\operatorname{pr}_{i} \in \operatorname{Hom}\left(S, S_{i}\right)$ be the projection. Let $\alpha \in \operatorname{Aut}(S)$ be such that $\alpha\left(\Omega_{1}\left(Z\left(S_{1}\right)\right)\right)=\Omega_{1}\left(Z\left(S_{1}\right)\right)$. Then for $i=1,2, \operatorname{pr}_{i}\left(\alpha\left(S_{i}\right)\right)=S_{i}$ and $\alpha\left(S_{i} Z\left(S_{3-i}\right)\right)=S_{i} Z\left(S_{3-i}\right)$.

We next look at semidirect products with normal dihedral subgroup.

Lemma B.2. Fix a finite 2-group $S$, and subgroups $\Delta \unlhd S$ and $T \leq S$. Assume that $S=T \Delta, T \cap \Delta=1, \Delta$ is dihedral of order $\geq 8$, and $\left[T, \Delta_{0}\right]=1$ for some dihedral subgroup $\Delta_{0} \leq \Delta$ of order 8 . Let $A, \Delta_{1}, \Delta_{2} \leq \Delta$ be the three subgroups of index two where $A$ is cyclic, and let $A_{0}=[\Delta, \Delta]$ be the subgroup of index two in $A$. Set $Z=Z(\Delta)$ for short. Fix $b \in \Delta_{0} \backslash A$.

(a) Assume $\varphi \in \operatorname{Hom}(\Delta, S)$ is such that $\varphi(Z)=Z$ and $\varphi(A) \unlhd S$. Then $\varphi\left(A_{0}\right)=A_{0}$, $\varphi(A) \leq T A$, and $\varphi(b) \in T A b$.

(b) Assume $\alpha \in \operatorname{Aut}(S)$ is such that $\alpha(Z)=Z$. Then either $\alpha$ sends each of the subgroups $T \Delta_{1}$ and $T \Delta_{2}$ to itself or it exchanges them.

Proof. Fix a generator $a \in A$; thus $A_{0}=\left\langle a^{2}\right\rangle$. Since $\Delta \unlhd S$ and $A$ is characteristic in $\Delta, A \unlhd S$. For all $t \in T,\left[t, \Delta_{0}\right]=1$ by assumption, so $[t, b]=1$, and $\left[t, a^{k}\right]=1$ if $\left|a^{k}\right| \leq 4$. Hence $t a t^{-1}=a^{4 j+1}$ for some $j$, so $[t, a] \in\left\langle a^{4}\right\rangle$. Thus $[T, \Delta] \leq\left\langle a^{4}\right\rangle$, and so $\left\langle T, a^{4}\right\rangle \unlhd S$.

(a) Assume $\varphi \in \operatorname{Hom}(\Delta, S)$ is such that $\varphi(Z)=Z$ and $\varphi(A) \unlhd S$. Since $A_{0}=[\Delta, \Delta]$, $\varphi\left(A_{0}\right) \leq[S, S] \leq T A_{0}$. If $\varphi\left(A_{0}\right) \leq\left\langle T, a^{4}\right\rangle$, then there are distinct elements $a^{i} \neq a^{j}$ in $A_{0}$ such that $\varphi\left(a^{i}\right), \varphi\left(a^{j}\right) \in T a^{k}$ for some $k$. Then $1 \neq a^{i-j}$ and $\varphi\left(a^{i-j}\right) \in T$, which is impossible since $Z \leq\left\langle a^{i-j}\right\rangle$ and $\varphi(Z)=Z \not \leq T$.

Thus $\varphi\left(a^{2}\right) \notin\left\langle T, a^{4}\right\rangle$, and the image of $\varphi(a)$ in $S /\left\langle T, a^{4}\right\rangle \cong D_{8}$ has order 4 . Hence $\varphi(a)=t a^{i}$ for some $t \in T$ and some odd $i$. Also, the image of $\varphi(b)$ in $S /\left\langle T, a^{4}\right\rangle$ must invert that of $\varphi(a)$, and so $\varphi(b) \in T A b$. Since $\varphi(A) \unlhd S, b\left(t a^{i}\right) b^{-1}=t a^{-i}$, and $\left(t a^{-i}\right)^{-1} t a^{i}=a^{2 i}$, we have $a^{2 i} \in \varphi(A)$. Thus $A_{0}=\left\langle a^{2 i}\right\rangle \leq \varphi(A)$, so $\varphi\left(A_{0}\right)=A_{0}$. This finishes the proof of (a). 
(b) Now assume $\alpha \in \operatorname{Aut}(S)$ is such that $\alpha(Z)=Z$. By (a), applied with $\varphi=\left.\alpha\right|_{\Delta}$, $\alpha(A) \leq T A$ and $\alpha\left(A_{0}\right)=A_{0}$. Hence $\alpha\left(\left\langle a^{4}\right\rangle\right)=\left\langle a^{4}\right\rangle$, and $\alpha$ induces an automorphism $\bar{\alpha}$ of $S /\left\langle a^{4}\right\rangle \cong T \times D_{8}$ which sends $Z\left(\Delta /\left\langle a^{4}\right\rangle\right)=A_{0} /\left\langle a^{4}\right\rangle$ to itself. By Proposition B.1, $\bar{\alpha}\left(T A_{0} /\left\langle a^{4}\right\rangle\right)=T A_{0} /\left\langle a^{4}\right\rangle$, and thus $\alpha\left(T A_{0}\right)=T A_{0}$. Since $\alpha(T A)=T A$, it now follows that $\bar{\alpha}$ either sends the two subgroups $T \Delta_{i} / T A_{0}$ of $S / T A_{0} \cong C_{2}^{2}$ to themselves $(i=1,2)$ or switches them.

The next lemma involves a similar situation.

Lemma B.3. Fix a finite 2-group $S$ with a normal dihedral or quaternion subgroup $\Delta \unlhd S$ of order $\geq 8$. Assume two of the three subgroups of index two in $\Delta$ are $S$-conjugate. Let $\Delta_{0} \leq \Delta$ be a dihedral or quaternion subgroup of order 8 , and set $T=C_{S}\left(\Delta_{0}\right)$. Let $A \unlhd \Delta$ be the cyclic subgroup of index two: the one which is normal in $S$ if $\Delta \cong Q_{8}$. Fix a generator $a \in A$, and choose $b \in \Delta_{0} \backslash A$. Let $Z \leq A_{0}<A$ be the subgroups of order two and index two, respectively. Then the following hold.

(a) $A \leq[S, S]$.

(b) $[S: T \Delta]=2$. For each $g \in S, g b g^{-1}=a^{i} b$ where $i$ is even if $g \in T \Delta$ and $i$ is odd if $g \in S \backslash T \Delta$.

(c) $T A_{0} \unlhd S, S / T A_{0} \cong D_{8}$, and $Z\left(S / T A_{0}\right)=T A / T A_{0}$.

(d) If $T$ is abelian, then $[S, S]$ is abelian.

(e) Assume $b_{1}, b_{2} \in S$ are in distinct cosets of $T A,\left[b_{1}, A\right]=A_{0}=\left[b_{2}, A\right], b_{i}^{2} \in A$, and $\left\langle A, b_{i}\right\rangle \unlhd S$. Then $\left\langle A, b_{1}, b_{2}\right\rangle$ is dihedral, semidihedral, or quaternion.

(f) Assume that $\Delta$ is dihedral and $Z$ is a direct factor of $T$. Let $\alpha \in \operatorname{Aut}(S)$ be such that $\alpha^{2} \in \operatorname{Inn}(S), \alpha(Z)=Z$, and $\alpha(T \Delta) \neq T \Delta$. Set $\widehat{\Delta}=\alpha(\Delta) \cdot \Delta$. Then $\widehat{\Delta}$ is dihedral, $\widehat{\Delta} \unlhd S,[\widehat{\Delta}: \Delta]=2, T \cap \widehat{\Delta}=Z$, and $T \widehat{\Delta}=S$.

Proof. By assumption, $\Delta \unlhd S$. Hence $A \unlhd S$ since $A$ is characteristic in $\Delta$, except when $\Delta \cong Q_{8}$ in which case $A$ was chosen to be the unique subgroup of index two in $\Delta$ normal in $S$.

(a) By assumption, the subgroups $\left\langle A_{0}, b\right\rangle$ and $\left\langle A_{0}, b a\right\rangle$ are $S$-conjugate. Hence there is $x \in S$ such that $c_{x}\left(b A_{0}\right)=b a A_{0}$, so $x b x^{-1}=b a^{i}$ for some odd $i$, and $A=\left\langle a^{i}\right\rangle \leq[S, S]$.

(b) By definition (and since $A=\langle a\rangle \unlhd S$ ),

$$
T=C_{S}\left(\Delta_{0}\right)=\left\{g \in S \mid g b g^{-1}=b, g a g^{-1}=a^{4 j+1} \text { some } j\right\} .
$$

Hence

$$
T \Delta=\left\{g \in S \mid c_{g} \in \operatorname{Aut}_{T}(\Delta) \operatorname{Inn}(\Delta)\right\}=\left\{g \in S \mid g b g^{-1}=a^{i} b \text { some } i \equiv 0(\bmod 2)\right\} .
$$

Since $\Delta \unlhd S$, this proves that $g b g^{-1}=a^{i} b$ with $i$ odd whenever $g \in S \backslash T \Delta$. Also, $T \Delta<S$ since $\left\langle A_{0}, b\right\rangle$ and $\left\langle A_{0}, a b\right\rangle$ are $S$-conjugate, and hence $[S: T \Delta]=2$.

(c) If $\Delta=\Delta_{0}$ has order 8 , then $T A_{0}=T=C_{S}(\Delta)$ is normal in $S$ since $\Delta \unlhd S$. So assume $|\Delta|>8$. Since $A \unlhd S, 1 \neq\left\langle a^{4}\right\rangle \unlhd S$. Also, $[T, A] \leq\left\langle a^{4}\right\rangle$ by (1), and hence $T \Delta /\left\langle a^{4}\right\rangle \cong(T / Z) \times D_{8}$. For $x \in S, c_{x}(T \Delta)=T \Delta$ by (b), so $c_{x}\left(T A_{0} /\left\langle a^{4}\right\rangle\right)=T A_{0} /\left\langle a^{4}\right\rangle$ by Proposition B.1 applied with $\alpha=c_{x} \in \operatorname{Aut}\left(T \Delta /\left\langle a^{4}\right\rangle\right)$. Thus $T A_{0} \unlhd S$.

Throughout the rest of the proof of the lemma, we set $\bar{S}=S / T A_{0}$, and let $\bar{P} \leq \bar{S}$ or $\bar{g} \in \bar{S}$ be the image of $P \leq S$ or $g \in S$. Thus $\overline{T \Delta}=\langle\bar{a}, \bar{b}\rangle \cong C_{2}^{2}$. 
For $x \in S \backslash T \Delta, c_{x}(\bar{a})=\bar{a}$ and $c_{x}(\bar{b})=\overline{a b}$ by (b). Thus $\bar{S} \cong D_{8}$, with center $\langle\bar{a}\rangle=\overline{T A}$.

(d) Assume $T$ is abelian. Since $A$ is cyclic, $\operatorname{Aut}(A)$ is abelian, and hence $[S, S]$ is in the kernel of the map $S \longrightarrow \operatorname{Aut}(A)$ induced by conjugation. Thus $[S, S] \leq C_{S}(A)$. Also, $[S, S] \leq T A$ since $T A$ is normal of index 4 in $S$. Since $T$ is abelian, $C_{T A}(A)$ is also abelian, and so is $[S, S]$.

(e) Fix $b_{1}, b_{2}$ as above, and set $\Delta_{i}=\left\langle A, b_{i}\right\rangle \unlhd S$ and $\widehat{\Delta}=\Delta_{1} \Delta_{2}$. Then $\widehat{\Delta} / A \cong C_{2}^{2}$ since the $\Delta_{i}$ are normal and contain $A$ with index two, so $\left(b_{1} b_{2}\right)^{2} \in A$. Also, $\left(\overline{b_{1}} \bar{b}_{2}\right)^{2}=\bar{a}$ in $\bar{S}$ since $\overline{b_{1}}$ and $\overline{b_{2}}$ are in distinct nonidentity cosets of $\bar{A}=Z(\bar{S})$ and have order 2 in $\bar{S}$, so $\left(b_{1} b_{2}\right)^{2}=a^{j}$ for some odd $j$. Thus $\widehat{A} \stackrel{\text { def }}{=}\left\langle b_{1} b_{2}\right\rangle$ is cyclic of index two in $\widehat{\Delta}$, conjugation by $b_{1}$ inverts the subgroup $A$ of index two in $\widehat{A}$, and hence $\widehat{\Delta}$ is dihedral, semidihedral, or quaternion (cf. [G, Theorem 5.4.4]).

(f) Now assume that $\Delta$ is dihedral, and that $T=T_{0} Z$ where $T_{0} \cap Z=1$. Let $\alpha \in \operatorname{Aut}(S)$ be such that $\alpha^{2} \in \operatorname{Inn}(S), \alpha(Z)=Z$, and $\alpha(T \Delta) \neq T \Delta$. Set $\Delta^{*}=\alpha(\Delta)$, and $\widehat{\Delta}=\Delta \Delta^{*}$. Since $\Delta$ and $\Delta^{*}$ are both normal in $S$ and are exchanged by $\alpha$ (since $\left.\alpha^{2} \in \operatorname{Inn}(S)\right), \widehat{\Delta}$ is normal in $S$.

Set $a_{*}=\alpha(a)$ and $b_{*}=\alpha(b)$. By (c), $T A_{0} \unlhd S$, and $\bar{S}=S / T A_{0}$ is dihedral of order 8 with center $T A / T A_{0}$.

Since $A \leq[S, S]$ by (a), $\alpha(A) \leq[S, S] \leq T A$. If $b_{*} \in T \Delta$, then $\alpha(\Delta) \leq T \Delta$, and by Lemma B.2(a) (applied with $\varphi=\left.\alpha\right|_{\Delta}$ and $T$ replaced by $T_{0}$ ), $b_{*} \in T_{0} A b$. So $\overline{b_{*}} \in\{\bar{b}, \overline{a b}\}$. Since $[T, b]=1$ (recall $\left.b \in \Delta_{0}\right),\left[\overline{\alpha(T)}, \bar{b}_{*}\right]=1$, so $\overline{\alpha(T)} \leq\langle\bar{a}, \bar{b}\rangle=\overline{T \Delta}$. Thus $\alpha(T) \leq T \Delta$, so $\alpha(T \Delta)=T \Delta$, and this contradicts our original assumption about $\alpha$.

Thus $b_{*} \notin T \Delta$. Hence by (b), $b_{*} b b_{*}^{-1}=a^{i} b$ for some odd $i$. Set $\widehat{a}=b_{*} b$; then $\widehat{a}^{2}=a^{i}$, and thus $\left\langle b_{*}, b\right\rangle=\langle\widehat{a}, b\rangle$ is a dihedral group which contains $\Delta$ with index two. Since $\alpha^{2} \in \operatorname{Inn}(S), \alpha\left(b_{*}\right)=\alpha^{2}(b)=a^{j} b$ for some $j$, and so $\alpha(\widehat{a})=a^{j} b b_{*}=a^{j}\left(b_{*} b\right)^{-1}=a^{j} \widehat{a}^{-1}$ is in $\langle\widehat{a}\rangle$. So $a_{*} \in\langle a\rangle,\left\langle b_{*}, b\right\rangle=\Delta \Delta^{*}$ is dihedral, and it contains $\Delta$ with index two. Also, $T \widehat{\Delta}=S$ since $[S: T \Delta]=2$ and $\widehat{\Delta} \not \leq T \Delta$, and $T \cap \widehat{\Delta}=T \cap \Delta=Z$ since $T \Delta \cap \widehat{\Delta}=\Delta$.

This will now be applied to prove the following lemma.

Lemma B.4. Fix a finite 2-group $S$, and subgroups $T, \Delta_{0} \leq S$ such that $\Delta_{0}$ is dihedral of order $8, T \cap \Delta_{0}=1$, and $\left[T, \Delta_{0}\right]=1$. Let $U, V \leq \Delta_{0}$ be the two noncyclic subgroups of order four, and set $Z=U \cap V=Z\left(\Delta_{0}\right)$. Assume $N_{S}(T U)=T \Delta_{0}, N_{S}\left(\Delta_{0}\right)>T \Delta_{0}$, and either $Z \leq Z(S)$ or $T$ contains no subgroup isomorphic to $D_{8}$. Let $\Delta \unlhd S$ be the normal closure of $\Delta_{0}$ in $S$. Then $\Delta$ is dihedral, $T \cap \Delta=1,[S: T \Delta]=2$, and all noncentral involutions in $\Delta$ are $S$-conjugate.

Proof. Case 1: Assume first that $Z \leq Z(S)$. Set $\Delta_{-1}=U$. We will construct subgroups $\Delta_{0}<\Delta_{1}<\cdots<\Delta_{m}<S$, all normalized by $T$, such that $\left[S: T \Delta_{m}\right]=2$, and such that for each $0 \leq i \leq m$,

(i) $\Delta_{i}$ is dihedral of order $2^{3+i}$;

(ii) $T \cap \Delta_{i}=1$ and $T \Delta_{i}=N_{S}\left(T \Delta_{i-1}\right)=N_{S}\left(\Delta_{i-1}\right)$; and

(iii) $N_{S}\left(\Delta_{i}\right)>T \Delta_{i}$. 
To simplify notation, we set $S_{i}=T \Delta_{i}$ whenever $\Delta_{i}$ has been defined.

When $i=0$, the only condition which is not immediate from the hypotheses is that $T \Delta_{0}=N_{S}(U)$. One inclusion is clear: $N_{S}(U) \geq T \Delta_{0}$ since $[U, T]=1$ and $U \unlhd \Delta_{0}$. If $g \in N_{N_{S}(U)}\left(T \Delta_{0}\right)$, then since $g$ normalizes $U$ and $T \Delta_{0}$ and $C_{T \Delta_{0}}(U)=T U$, $g \in N_{S}(T U)=T \Delta_{0}$. Thus $N_{N_{S}(U)}\left(T \Delta_{0}\right)=T \Delta_{0}$, and so $N_{S}(U)=T \Delta_{0}$ by Lemma A.1.

Assume, for some $i \geq 0$, that we have constructed $\Delta_{i}$ which satisfies (i)-(iii). If $\left[S: S_{i}\right]=2$ (recall $S_{i}=T \Delta_{i}$ ), then $\Delta_{i} \unlhd S$ since $N_{S}\left(\Delta_{i}\right)>S_{i}$. For $g \in S \backslash S_{i}$, $g \notin N_{S}\left(\Delta_{i-1}\right)=S_{i}$ implies that $g$ exchanges the two conjugacy classes of noncentral involutions in $\Delta_{i}$, and hence the noncentral involutions in $\Delta_{i}$ are all $S$-conjugate. If $i>0$, then the normal closure of $\Delta_{0}$ in $\Delta_{i}$ is $\Delta_{i-1}$ which is not normal in $S$, and thus $\Delta_{i}$ is the normal closure of $\Delta_{0}$ in $S$ (this is trivial if $i=0$ ). So the subgroup $\Delta=\Delta_{i}$ satisfies the conditions in the statement of the lemma.

Now assume $\left[S: S_{i}\right] \geq 4$. Set $S_{i+1}=N_{S}\left(\Delta_{i}\right)>S_{i}$. Let $\Delta_{i-1}$ and $\Delta_{i-1}^{*}$ be the two dihedral subgroups of index two in $\Delta_{i}$. Since $S_{i}=N_{S}\left(\Delta_{i-1}\right)$ and $\Delta_{i} \unlhd S_{i+1}$, conjugation by any element of $S_{i+1} \backslash S_{i}$ exchanges $\Delta_{i-1}$ with $\Delta_{i-1}^{*}$. The product of any two elements of $S_{i+1} \backslash S_{i}$ thus lies in $S_{i}$, so $\left[S_{i+1}: S_{i}\right]=2$, and $S_{i} \unlhd S_{i+1}<S$.

For each $x \in N_{S}\left(S_{i}\right)$, since $c_{x}(Z)=Z$ by assumption $(Z \leq Z(S))$, Lemma B.2(b) implies that $c_{x}$ either leaves $T \Delta_{i-1}$ and $T \Delta_{i-1}^{*}$ invariant or exchanges them; and leaves them invariant only if $x \in N_{S}\left(T \Delta_{i-1}\right)=S_{i}$. Thus $\left[N_{S}\left(S_{i}\right): S_{i}\right]=2$. Since $S_{i} \unlhd S_{i+1}$ with index two, this implies $N_{S}\left(S_{i}\right)=S_{i+1}$.

Choose any $g \in N_{S}\left(S_{i+1}\right) \backslash S_{i+1}$ such that $g^{2} \in S_{i+1}$, and set $\alpha=c_{g} \in \operatorname{Aut}\left(S_{i+1}\right)$. Then $\alpha(Z)=Z$ since $Z \leq Z(S)$, and $\alpha\left(S_{i}\right) \neq S_{i}$ since $g \notin S_{i+1}=N_{S}\left(S_{i}\right)$. Also, $C_{S}\left(\Delta_{0}\right)=T Z$ since $N_{S}(U)=T \Delta_{0}$ by (ii) when $i=0$ (and since $C_{S}\left(\Delta_{0}\right) \leq C_{S}(U) \leq$ $N_{S}(U)$ ). The hypotheses of Lemma B.3(f) thus hold (but where $T$ in Lemma B.3 corresponds to $T Z$ here). So if we define $\Delta_{i+1}=\Delta_{i} \cdot \alpha\left(\Delta_{i}\right)$, then $\Delta_{i+1} \unlhd S_{i+1}$ is dihedral, $\left[\Delta_{i+1}: \Delta_{i}\right]=2, T \cap \Delta_{i+1}=1$, and $T \Delta_{i+1}=S_{i+1}$. Thus (i) and (ii) hold, (iii) holds since $g \in N_{S}\left(\Delta_{i+1}\right)$, and this finishes the induction step in the proof.

Case 2: Now assume $Z \not Z Z(S)$, and set $S_{*}=C_{S}(Z)<S$. By Case 1, $S_{*}$ has the form described in the lemma: it contains a normal dihedral subgroup $\Delta \unlhd S_{*}$ (the normal closure of $\Delta_{0}$ in $\left.S_{*}\right), T \cap \Delta=1$, and $\left[S_{*}: T \Delta\right]=2$. We prove that $T$ contains a subgroup isomorphic to $D_{8}$, contradicting the assumptions.

Choose any $g \in N_{S}\left(S_{*}\right) \backslash S_{*}$ such that $g^{2} \in S_{*}$, and set $\beta=c_{g} \in \operatorname{Aut}\left(S_{*}\right)$. Then $\beta(Z) \neq Z$. Assume first that $\beta(\Delta) \not \leq T \Delta$, and choose $x \in \beta(\Delta) \backslash T \Delta$. Let $A \unlhd \Delta$ be the cyclic subgroup of index two $\left(A \unlhd S_{*}\right)$, and choose $b \in U \backslash Z$. (Recall $C_{2}^{2} \cong U \leq$ $\Delta_{0}$.) If $c_{x}(b) \in \Delta \backslash A$ is $\Delta$-conjugate to $b$, then $c_{a x}(U)=U$ for some $a \in A$, which is impossible since we showed in the proof of Case 1 that $N_{S}(U)=T \Delta_{0}$. Thus $b$ is not $\Delta$-conjugate to $x b x^{-1}$, so $[x, b]$ generates $A$, and hence $A \leq \beta(\Delta)$ since $x \in \beta(\Delta) \unlhd S_{*}$. Since $A$ is cyclic of order $\geq 4$, this is possible only if $\beta(A)=A$, which is impossible since $\beta(Z) \neq Z$.

Thus $\beta(\Delta) \leq T \Delta$. Let $\psi \in \operatorname{Hom}(\Delta, T)$ be the composite $\Delta \stackrel{\beta}{\longrightarrow} T \Delta \longrightarrow T \Delta / \Delta \cong$ $T$. Since $\beta(Z) \neq Z$ and $\beta(Z) \leq Z(T \Delta), \beta(Z) \not \leq \Delta$, and hence $Z \not \leq \operatorname{Ker}(\psi)$. Since any nontrivial normal subgroup of $\Delta$ contains $Z$, this implies that $\psi$ is injective, and thus that $T$ contains a subgroup isomorphic to $D_{8}$.

We also need the following corollary to Lemma B.4. 
Lemma B.5. Fix a finite 2-group $S$, and an abelian subgroup $P \leq S$ such that $\left|N_{S}(P) / P\right|=2$ and $P \unlhd S$. Assume either

(a) $\operatorname{Aut}_{S}(P) \not O_{2}(\operatorname{Aut}(P))$, or

(b) there is a direct factor $U \leq P$ such that $U \cong C_{2}^{2}$ and $1 \neq\left[N_{S}(P), U\right] \leq U$.

Then there are subgroups $\Delta_{0} \leq \Delta \unlhd S$ such that $\Delta$ is dihedral, $\Delta_{0} \cong D_{8}, C_{S}\left(\Delta_{0}\right) \leq P$, and the noncentral involutions in $\Delta$ are all $S$-conjugate. In case (b), $\Delta$ can be taken to be the normal closure of $U$ in $S$.

Proof. Set $\widehat{P}=N_{S}(P)<S$, fix $x \in N_{S}(\widehat{P}) \backslash \widehat{P}$ such that $x^{2} \in \widehat{P}$, and set $Q=x P x^{-1}$. Set $Z=[\widehat{P}, \widehat{P}]$. Since $\widehat{P}$ is nonabelian, and $P, Q$ are distinct abelian subgroups of index two, Lemma A.6(c,a) implies that $|Z|=2$, and $Z(\widehat{P})=P \cap Q$ has index 2 in $P$ and in $Q$. Also, $Z \leq Z(\widehat{P})$.

We first show that (a) implies (b). Consider the subgroups

$$
\Theta_{1}=\{\alpha \in \operatorname{Aut}(P) \mid[\alpha, P] \leq \operatorname{Fr}(P)\} \quad \text { and } \quad \Theta_{2}=\left\{\alpha \in \operatorname{Aut}(P)|\alpha|_{\Omega_{1}(P)}=\operatorname{Id}\right\} .
$$

Both are normal in $\operatorname{Aut}(P)$, and they are 2-subgroups by Lemma A.2 and [G, Theorem 5.2.4], respectively. Thus $\Theta_{1} \Theta_{2} \leq O_{2}(\operatorname{Aut}(P))$. Since $\operatorname{Aut}_{\widehat{P}}(P)=\operatorname{Aut}_{S}(P) \not$ $O_{2}(\operatorname{Aut}(P))$ by assumption, $\operatorname{Aut}_{\widehat{P}}(P) \not{\not} \Theta_{1} \Theta_{2}$. Hence for $y \in \widehat{P} \backslash P, Z=[y, P] \not{\not}$ $\operatorname{Fr}(P)$ and $Z(\widehat{P})=C_{P}(y) \nsupseteq \Omega_{1}(P)$.

Thus there are $T<Z(\widehat{P})$ and $g \in P \backslash Z(\widehat{P})$ such that $|g|=2$ and $P=Z \times T \times\langle g\rangle$ $\left(T=\operatorname{Ker}(f)\right.$ for any $f: Z(\widehat{P}) \rightarrow Z(\widehat{P}) / \operatorname{Fr}(Z(\widehat{P})) \rightarrow C_{2}$ with $\left.Z \not \mathbf{K e r}(f)\right)$. Set $U=\langle Z, g\rangle$. Then $U \cong C_{2}^{2}, P=T U$, and $T \cap U=1$. So (b) holds.

Now assume (b). Thus $P=T U$ where $U \cong C_{2}^{2}, T \cap U=1$, and $Z=[\widehat{P}, \widehat{P}] \leq U$. By the argument used in the last paragraph, we can assume that $T<Z(\widehat{P})$.

Set $V=x U x^{-1}$ and $\Delta_{0}=U V$. Then $\Delta_{0} \cong D_{8}$ since $[U, V]=Z$ and $U \cong V \cong C_{2}^{2}$, $T \cap \Delta_{0}=T \cap Z=1$, and $\left[T, \Delta_{0}\right] \leq[T, \widehat{P}]=1$ since $T \leq Z(\widehat{P})$. Also, $N_{S}(T U)=$ $N_{S}(P)=\widehat{P}=T \Delta_{0}$, and $N_{S}\left(\Delta_{0}\right)>T \Delta_{0}$ since $x \in N_{S}\left(\Delta_{0}\right) \backslash T \Delta_{0}$. Since $T$ is abelian, it contains no subgroup isomorphic to $D_{8}$. So by Lemma B.4, the normal closure $\Delta$ of $\Delta_{0}$ in $S$ is dihedral, and all noncentral involutions in $\Delta$ are $S$-conjugate.

Set $R=N_{C_{S}\left(\Delta_{0}\right)}(Z(\widehat{P}))$. Then $R$ normalizes $Z(\widehat{P}) U=P$, so $R \leq N_{S}(P)=\widehat{P}$. Hence $R \leq C_{\widehat{P}}\left(\Delta_{0}\right)=Z(\widehat{P})$, so by Lemma A.1, $C_{S}\left(\Delta_{0}\right)=Z(\widehat{P}) \leq P$.

\section{REFERENCES}

[AOV] K. Andersen, B. Oliver, \& J. Ventura, Reduced, tame, and exotic fusion systems, Proc. London Math. Soc. (to appear)

[A1] M. Aschbacher, Finite group theory, Cambridge Univ. Press (1986)

[A2] M. Aschbacher, A 2-local characterization of $M_{12}$, Illinois J. Math. 47 (2003), 31-47

[AKO] M. Aschbacher, R. Kessar, \& B. Oliver, Fusion systems in algebra and topology, Cambridge Univ. Press (to appear)

[BLO1] C. Broto, R. Levi, \& B. Oliver, Homotopy equivalences of $p$-completed classifying spaces of finite groups, Invent. Math., 151 (2003), 611-664.

[Bd] H. Bender, Transitive Gruppen gerader Ordnung, in denen jede Involution genau einen Punkt festlässt, J. Algebra 17 (1971), 527-554

[5a2] C. Broto, N. Castellana, J. Grodal, R. Levi, \& B. Oliver, Extensions of p-local finite groups, Trans. Amer. Math. Soc. 359 (2007), 3791-3858 
[BMO] C. Broto, J. Møller, \& B. Oliver, Equivalences between fusion systems of finite groups of Lie type, Jour. Amer. Math. Soc. (to appear)

[Bu] W. Burnside, The theory of groups of finite order, Cambridge Univ. Press (1897)

[CE] H. Cartan \& S. Eilenberg, Homological algebra, Princeton Univ. Press (1956)

[Car] R. Carter, Simple groups of Lie type, Wiley (1972)

[CF] R. Carter \& P. Fong, The Sylow 2-subgroups of the finite classical groups, J. Algebra 1 (1964), 139-151

[Fn] P. Fan, Amalgams of prime index, J. Algebra 98 (1986), 375-421

[Gd1] D. Goldschmidt, A conjugation family for finite groups, J. Algebra 16 (1970), 138-142

[Gd2] D. Goldschmidt, Automorphisms of trivalent graphs, Annals of Math. 111 (1980), 377-406

G] D. Gorenstein, Finite groups, Harper \& Row (1968)

[GH] D. Gorenstein \& K. Harada, Finite groups whose 2-subgroups are generated by at most 4 elements, Memoirs Amer. Math. Soc. 147 (1974)

[Gr] R. Griess, Schur multipliers of finite simple groups of Lie type, Trans. Amer. Math. Soc. 183 (1973), 355-421

[Hu] B. Huppert, Endliche Gruppen I, Springer-Verlag (1967)

[Mz] P. Mazet, Sur les multiplicateurs de Schur des groupes de Mathieu, J. Algebra 77 (1982), $552-576$

[O1] B. Oliver, Splitting fusion systems over 2-groups, proceedings of the 2009 Skye conference on algebraic topology, group theory and representation theory, Proc. Edinburgh Math. Soc. (to appear).

[OV] B. Oliver \& J. Ventura, Saturated fusion systems over 2-groups, Trans. Amer. Math. Soc. 361 (2009), 6661-6728.

[Pg1] L. Puig, The hyperfocal subalgebra of a block, Invent. math. 141 (2000), 365-397

[Pg2] L. Puig, Frobenius categories, J. Algebra 303 (2006), 309-357

[Sch] J. Schur, Über die Darstellung der endlichen Gruppen durch gebrochene lineare Substitutionen, J. reine angew. Math. 127 (1904), 20-50

[Sz1] M. Suzuki, Group theory I, Springer-Verlag (1982)

[Sz2] M. Suzuki, Group theory II, Springer-Verlag (1986)

[Ta] D. Taylor, The geometry of the classical groups, Heldermann (1992)

[Wi] R. Wilson, The geometry and maximal subgroups of the simple groups of A. Rudvalis and J. Tits, Proc. London Math. Soc. 48 (1984), 533-563

Center for Mathematical Sciences, LTH, Box 118, 22100 Lund, Sweden

E-mail address: kksa@maths.1th.se

LAGA, Institut Galilée, Av. J-B Clément, 93430 Villetaneuse, France

E-mail address: bobol@math.univ-paris13.fr

Departamento de Matemática, Instituto Superior Técnico, Av. Rovisco Pais, 1049 001 Lisboa, Portugal

E-mail address: jventura@math.ist.utl.pt 